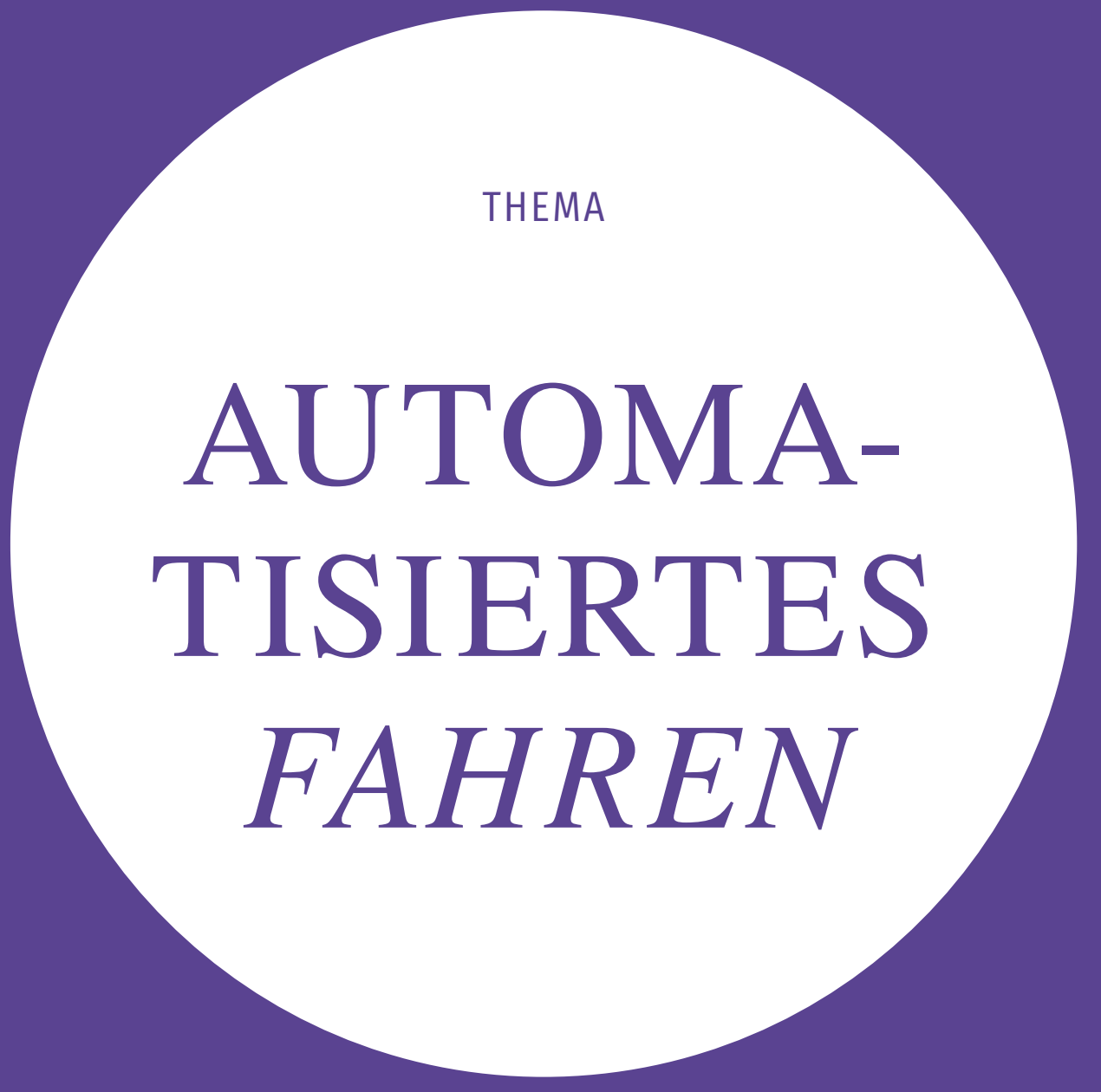

Die Mobilitätsforschung erwartet, dass hoch- oder vollautomatisierte Fahrzeuge in absehbarer Zeit zugelassen werden - auch wenn in der Öffentlichkeit weiterhin Skepsis herrscht. Aber wird sich die Automatisierung des Straßenverkehrs als Fluch oder als Segen für Konzepte nachhaltiger Mobilität erweisen? Ein TATuP-Thema herausgegeben von Torsten Fleischer und Jens Schippl. 


\title{
Automatisiertes Fahren
}

\author{
Fluch oder Segen für nachhaltige Mobilität?
}

Torsten Fleischer, Institut für Technikfolgenabschätzung und Systemanalyse (ITAS), Karlsruher Institut für Technologie (KIT),

Karlstraße 11, 76133 Karlsruhe (torsten.fleischer@kit.edu)

Jens Schippl, Institut für Technikfolgenabschätzung und Systemanalyse (ITAS), Karlsruher Institut für Technologie (KIT) (jens.schippl@kit.edu)

Dieses TATuP-Thema präsentiert erste Perspektiven, Indiziensammlungen und Diskussionsbeiträge zum Themenfeld des automatisierten Fahrens, das seit einigen Jahren ein wichtiger Gegenstand der Mobilitätsforschung geworden ist. Auch wenn in der Öffentlichkeit weiterhin verbreitet Skepsis hinsichtlich der technischen und organisatorischen Reife herrscht, dominiert in der Fachwelt die Erwartung, dass hochoder vollautomatisierte Fahrzeuge in absehbarer Zeit zugelassen werden. Aber wird sich die Automatisierung des Straßenverkehrs als Fluch oder als Segen für Konzepte nachhaltiger Mobilität erweisen? Die Technikfolgenabschätzung (TA) sieht sich dabei mit einem für die Disziplin nicht untypischen Dilemma konfrontiert: Einerseits lässt sich angesichts der vielen Unwägbarkeiten noch wenig Belastbares über mögliche Technikfolgen sagen. Andererseits hat die Technologie erhebliches Transformationspotenzial, sodass ein "rechtzeitiges" Gestalten ihrer Entwicklung gesellschaftlich wünschenswert scheint.

\section{Automated Driving.}

Blessing or curse for a sustainable mobility?

This special topic of TATUP presents a collection of initial perspectives, first indications and discussions on the topic of automated driving, which has become an important subject of mobility research in recent years. Even though there is still widespread public scepticism about its technical and organizational maturity, many experts expect vehicles with high or full driving automation to be deployed in the foreseeable future. But will road traffic automation be a blessing or a curse for future sustainable mobility concepts? Technology Assessment (TA) is confronted with a dilemma that is not untypical for this discipline: On the one hand, in view of the many uncertainties, possible implications of the new technology can so far only vaguely be described. On the other hand, the technology has considerable transformation potential, so that a timely shaping of its development seems socially desirable.

Keywords: automated driving, self-driving cars, sustainable mobility

This is an article distributed under the terms of the Creative Commons Attribution License CCBY 4.0 (https://creativecommons.org/licenses/by/4.0/)

https://doi.org/10.14512/tatup.27.2.11
Mobilitätsysteme mit den entsprechenden Infrastrukturen gehören $\mathrm{zu}$ den entscheidenden Faktoren für die Lebensqualität und wirtschaftliche Entwicklung in modernen Gesellschaften. Technische Entwicklungen und ihre Folgen, aber auch organisationale oder soziale Innovationen oder Planungen waren und sind von entsprechend großem gesellschaftlichem Interesse und somit auch immer Gegenstand von Technikfolgenabschätzung (TA), Foresight und verwandten Aktivitäten. Über viele Jahrzehnte gab es teilweise sehr kontroverse Debatten darüber, wie jeweils zukünftige Mobilität aussehen werde (und aussehen sollte), welche Folgen damit verbunden sein könn(t)en und wie diese Folgen zu bewerten seien. Spätestens seit den 1970er-Jahren wurde in Deutschland und in anderen Ländern das Paradigma der autogerechten Planung ersetzt oder zumindest ergänzt (auch darüber lässt sich sicherlich streiten) durch umweltorientiertere Planungsansätze, die auch andere Verkehrsträger wie Öffentliche Verkehrsmittel (ÖV) und Fahrrad stärker in den Blick nehmen. Seit einigen Jahrzehnten ist das Konzept „,nachhaltiger Entwicklung“ aus Verkehrsentwicklungsplänen deutscher Städte nicht mehr wegzudenken - auch wenn es oft sehr unterschiedliche Vorstellungen dazu gibt, wie genau ein nachhaltiges Verkehrssystem aussehen soll und wie ein solches erreicht werden kann.

Ein Element dieser Auseinandersetzungen betrifft die Frage, welche Rolle welche Technik in einem nachhaltigen Verkehrssystem spielen könne, spielen dürfe oder spielen solle. „Saubere“ Antriebstechniken für den motorisierten Verkehr stehen seit langer Zeit im Fokus, auch wenn die aktuelle politische und mediale Favoritin hier immer mal wieder wechselt. Technische und organisatorische Verbesserungen für den nichtmotorisierten Verkehr, vor allem in urbanen Räumen, sind seit einigen Jahren ein weiteres prominentes Diskussionsfeld. Lange Zeit ein Schattendasein hingegen fristeten Entwicklungen im Bereich der Informations- und Kommunikationstechniken für verkehrliche Anwendungen, auch wenn sie spätestens seit den 1990er-Jahren eine große - und weiter wachsende - Bedeutung im wissenschaftlichen Diskurs erlangten. Unter Stichworten wie Verkehrstelematik oder Intelligent Transport Systems wurden viele Ansätze entwickelt und auch umgesetzt, um Abläufe im Verkehrssystem zu optimieren und auch nachhaltiger zu machen. Verkehrsinformationssysteme verbesserten die Qualität der Pla- 
nungs- und Informationsmöglichkeiten für die Nutzer der verschiedenen Verkehrsmittel - mit der weiten Verfügbarkeit von mobilen Endgeräten und entsprechenden Dienstangeboten immer mehr auch während der Fahrt.

$\mathrm{Zu}$ den technischen Optionen ist in den letzten Jahren die Automatisierung von Fahraufgaben in Straßenfahrzeugen hinzugetreten, das sogenannte automatisierte oder gar ,autonome“ Fahren. In kurzer Zeit hat es sich zu einem Top-Thema in Industrie, Politik, Wissenschaft und Medien entwickelt. Alle großen Automobilhersteller, prominente Unternehmen der IT-Branche, zahlreiche Start-ups sowie viele Universitäten und öffentliche Forschungseinrichtungen arbeiten an Techniken und Konzepten trachtet werden können) spielt eine zentrale Rolle für das soziale Miteinander in modernen Gesellschaften. Eine Transformation des Mobilitätssystems hätte demnach tiefgreifenden Einfluss auf unseren gesellschaftlichen Alltag - technischer Wandel, der dies befördern könnte, sollte mithin Gegenstand öffentlicher und politischer Diskussion sein. Eine solche Debatte findet allerdings bisher erst in Ansätzen statt und wird momentan auch eher als Ermöglichungsdiskurs denn als Reflexionsdiskurs geführt.

Ein Grund dafür soll im Folgenden kurz angerissen werden: Hinter den Überschriften ,Automatisiertes Fahren“ oder „Autonomes Fahren“" verbergen sich sehr unterschiedliche technische Realisierungen von Fahrzeugautomatisierungen mit unterschied-

\section{Die hochkomplexe Aufgabe der Automatisierung von Fahraufgaben in Straßenfahrzeugen scheint vielen Entwicklern inzwischen beherrschbar und auch wirtschaftlich und politisch attraktiv.}

dafür. Beinahe im Wochenrhythmus wird von neuen Entwicklungsfortschritten (und inzwischen bedauerlicherweise auch von Unfällen) berichtet. Dabei scheinen diese aktuellen Entwicklungen zunächst vor allem getrieben von neuen technischen Möglichkeiten bei den Informations- und Kommunikationstechniken. Die hochkomplexe Aufgabe der Automatisierung von Fahraufgaben in Straßenfahrzeugen scheint vielen Entwicklern inzwischen beherrschbar und auch wirtschaftlich und politisch attraktiv. Automatisierung und Vernetzung von zukünftigen Verkehrsträgern, so die Erwartungen der Protagonisten, könnten dazu beitragen, mehr Sicherheit im Straßenverkehr zu gewährleisten, den Zugang zu Mobilität zu erleichtern und - in Kombination mit emissionsarmen oder lokal emissionsfreien Antriebstechniken - die negativen Begleiterscheinungen der Mobilität zu reduzieren (acatech 2015). Erhöhung der Effizienz des Verkehrssystems, höherer Komfort für die Nutzer sowie neue Mobilitätsoptionen für Personengruppen, die aus Alters- oder Gesundheitsgründen von individualisierter motorisierter Mobilität noch oder wieder ausgeschlossen sind, sind weitere typischerweise formulierte Leistungsversprechen. Als längerfristiges Entwicklungsziel wird angestrebt, (Straßen-)Fahrzeuge nicht nur umfassend zu automatisieren, sondern sie so weit zu entwickeln, dass sie tatsächlich zu eigenständigen, ohne unmittelbare menschliche Unterstützung agierenden Teilnehmern im Straßenverkehr werden können. Ob, und wenn ja in welchem Umfang das in welchem Zeitraum realisierbar wird, ist heute durchaus umstritten. Ließe sich dieses allerdings realisieren, könnte Automatisierung völlig neue verkehrstechnische und verkehrsorganisatorische Lösungen ermöglichen und dadurch sogar zu einer Transformation des gesamten heutigen Mobilitätssystems führen (Schippl et al. 2018).

Mobilität von Personen (aber auch von Gütern und Informationen, die im Rahmen dieses Textes leider nicht weiter be- lichem technischen Reifegrad, unterschiedlicher Leistungsfähigkeit, unterschiedlicher Aufgabenverteilung zwischen Mensch und Maschine (mit unterschiedlichen Konsequenzen für Verantwortlichkeit, Haftung und ethische Fragestellungen) sowie unterschiedlichem Potenzial für die Umsetzung neuer Systemkonzepte. Die Bundesanstalt für Straßenwesen (BASt 2012) sowie die Society of Automotive Engineers (SAE 2016) haben jeweils Nomenklaturen für die Beschreibung unterschiedlicher Ausprägungen von Fahrzeugautomatisierung entwickelt, die diese anhand von Stufen (bzw. Level) beschreiben. Für diese ist zunächst festzuhalten, dass sie nicht alle Formen heutiger und zukünftiger Fahrzeugautomatisierung beschreiben. Sie blenden beispielsweise in unfallgeneigten Situationen temporär intervenierende Funktionen wie automatische Blockierverhinderer (ABS) oder Elektronische Stabilitätskontrolle (ESC/ESP) aus und decken lediglich kontinuierlich automatisierende Funktionen in der Fahrzeugsteuerung ab. Bei diesen wiederum lassen sich - vereinfacht - vier Gruppen unterscheiden:

1. Automatisierungen, die die ständige Mitwirkung des Fahrers in der Fahrzeugsteuerung und/oder ihrer Überwachung erfordern (Stufe/Level 0-2),

2. Automatisierungen, bei denen der Fahrer das System nicht mehr dauerhaft überwachen muss, aber zugleich in der Lage zu sein hat, die Fahraufgabe (beispielsweise nach Aufforderung durch das System oder eigenem Ermessen) zu übernehmen (Stufe/Level 3 ,hochautomatisiert“/,,conditional driving automation"),

3. Automatisierungen, die die Fahraufgabe auf bestimmten StraBentypen, in bestimmten Geschwindigkeitsbereichen und/ oder unter bestimmten Umfeldbedingungen ohne Mitwirkung eines menschlichen Fahrers bewältigen können (Stufe/Level 4 ,vollautomatisiert“",high driving automation“), sowie 
4. Automatisierungen, die die Fahraufgabe vollumfänglich unter allen Bedingungen (vermutlich präziser: unter solchen, die auch für einen menschlichen Fahrer lösbar sind) bewältigen können (Stufe/Level 5 ,fahrerlos“/,full driving automation“).

Level-2-Systeme sind heute marktgängig und in Fahrzeugen zahlreicher Hersteller zu finden, auch das als „Autopilot“ beworbene System eines amerikanischen Unternehmens ist hierzu zu zählen. Die Novelle des Straßenverkehrsgesetzes im vergangenen Jahr ermöglicht, dass Level-3-Systeme in Deutschland verkauft werden können. Fahrzeuge, die unter sehr kontrollierten Randbedingungen eine Fahraufgabe vollautomatisiert bewältigen können, befinden sich in der Demonstration. Level-5-Fahrzeuge sind aus heutiger Sicht noch Zukunftsmusik. Level-3-Automatisierungen erfordern immer noch einen fahrtauglichen Fahrer an Bord, eignen sich also nicht für die Umsetzung von Konzepten, die neue Mobilitätsoptionen für von individualisierter motorisierter Mobilität ausgeschlossene Personengruppen eröffnen. Level-4-Konzepte unterliegen in der Regel räumlichen und/oder zeitlichen Einschränkungen, die ihre verkehrliche Flexibilität begrenzen und beim Verlassen ihres vordefinierten Aktionsraums menschlicher Mitwirkung bedürfen. Vollwertige Vehicle-on-demand-Systeme, die den klassischen Pkw zu substituieren in der Lage sein werden oder zentraler Baustein neuer Angebote im Personenverkehr sein könnten, werden wohl eine Level-5-Automatisierung (oder alternativ eine in einem ganz weiten Rahmen voll funktionale Level-4-Automatisierung) voraussetzen. Bedauerlicherweise verzichtet die mediale und öffentliche Debatte in der Regel auf diese Differenzierungen, was einerseits überzogene Erwartungen befördert, andererseits auch Sorgen hinsichtlich der Folgen der verbreiteten Einführung von automatisierten Fahrzeugen Vorschub leistet.

Es wird sicherlich noch einige Zeit strittig bleiben, ob die letztgenannte Entwicklungsstufe des automatisierten Fahrens, die breite Einsetzbarkeit fahrerloser Fahrzeuge im Individualwie im öffentlichen Personenverkehr, bereits in wenigen Jahren Realität sein wird oder ob man bis dahin eher noch in Jahrzehnten rechnen muss. Es steht jedoch zu erwarten, dass diese Entwicklung - sollte sie in absehbarer Zeit möglich werden - mit erheblichen Konsequenzen für Verkehrssystem, Mobilitätsmuster, Gesellschaft, Wirtschaft und Umwelt einhergehen würde. Angesichts der langen Planungshorizonte und Reaktionszeiten von Verkehrspolitik scheint es darum leichtfertig, sich bei der Entwicklung verkehrspolitischer Strategien nicht mit diesem Phänomen auseinanderzusetzen. Bisher findet man dies nur selten - in dem auf klare Ziele ausgerichteten White Paper der Europäischen Union von 2011 (CEC 2011) ebenso wenig wie in den aktuellen Verkehrsentwicklungsplänen fast aller deutschen Städte.

Dies ist angesichts vieler offener Fragestellungen in diesem Kontext vielleicht auch nicht verwunderlich - etwa jener, in welchem Umfang Fahrzeugautomatisierung faktisches Mobilitätsverhalten verändert, ob sie Arbeitsplätze im Speditions- und Logistikbereich gefährdet, ob wir Menschen in Zukunft im verkehr- lichen Alltag mit Fahrrobotern werden kommunizieren lernen müssen oder ob das Führen von Kraftfahrzeugen durch Menschen in Zukunft aus Sicherheitsgründen verboten sein wird. Dennoch scheint es im Sinne einer anticipatory governance angeraten, sich frühzeitig mit diesen möglichen Folgen auseinanderzusetzen, unter anderem um gesellschaftliche Positionsbestimmung zu ermöglichen und politische Gestaltungsprozesse zu informieren.

Gleiches gilt natürlich auch für die Frage nach den - wissenschaftlich belegbaren - Effekten von automatisiertem Fahren für ein nachhaltiges Verkehrssystem. Nicht zuletzt angesichts der vielen Unsicherheiten auf der technischen und organisatorischen Seite steht die Diskussion hierzu noch sehr am Anfang. Im Lichte der vorstehenden Argumentation wird in diesem TATuP-Thema dennoch der Versuch unternommen, erste diesbezügliche Perspektiven, Indiziensammlungen und Diskussionsbeiträge zusammenzutragen und zu präsentieren.

\section{Beiträge in diesem Heft}

Karsten Weber und Sonja Haug fügen dem inzwischen durchaus umfangreichen Fundus an empirischen Untersuchen hinsichtlich der Wahrnehmung automatisierten Fahrens sowie des Umgangs damit durch die prospektiven Nutzerinnen und Nutzer eine weitere Perspektive hinzu. Sie berichten über eine explorative Pilotstudie, in deren Rahmen das Fahrverhalten unterschiedlicher Personengruppen vergleichend untersucht und auf dieser Grundlage Typisierungen ihrer Fahrstile vorgenommen wurden. Die Ergebnisse weisen unter anderem darauf hin, dass sich die Probandinnen und Probanden in Bezug auf Sicherheit und Effizienz ihrer Fahrweise selbst höher als die Beobachter einschätzen. Dies galt für alle Fahrtypen, in besonderem Maße aber für den Fahrtypus der Sportlichen, die sich in Bezug auf Sicherheit am meisten überschätzen. Selbstüberschätzung im Hinblick auf eine sichere und ressourcensparende Fahrweise scheint somit relativ weit verbreitet zu sein. Ließe sich diese Beobachtung empirisch weiter härten, wiese sie auf ein Spannungsfeld in der Parametrisierung zukünftiger Fahrzeugautomatisierungen hin. Legt man deren Fahrverhalten so aus, dass ein besonderes Augenmerk auf Ressourcensparsamkeit und Sicherheit gelegt würde, widersprächen sie (zumindest anfangs) den Verhaltenserwartungen vieler Nutzerinnen und Nutzer. Passt man sie hingegen an die Erwartungen der Nutzerinnen und Nutzer an, blieben potenzielle Sicherheits- und Effizienzgewinne (und damit Nachhaltigkeitspotenziale) ungenutzt.

Nachhaltigkeitsbeziehbare Effekte entstehen mit von Menschen gesteuerten Fahrzeugen unter anderem durch Unfälle, Staus und das Parken in Ballungsräumen. Michael Roos und Marvin Siegmann gehen in ihrer Studie der Frage nach, in welchem Umfang diese durch den Einsatz von selbstfahrenden Autos vermieden werden könnten und welche gesamtwirtschaftlichen Kosteneinsparungen für Deutschland daraus erwachsen würden. Im Zuge ihrer Abschätzungen - die beim gegenwärtigen 
Stand des Wissens noch darüber hinwegsehen müssen, dass sich im Zuge der Markteinführung selbstfahrender Fahrzeuge Preise, Kosten und Nutzungsverhalten ändern werden und diese Größen hier nur einfach substitutiv betrachten - ermitteln sie, dass bei einem Marktanteil von 50\% selbstfahrender Fahrzeuge jährlich knapp 900.000 Unfälle vermieden, mehr als 1.000 Leben gerettet und somit fast $25 \mathrm{Mrd}$. Euro eingespart werden könnten. Die Vermeidung von Unfällen trägt etwa zur Hälfte zu dieser finanziellen Gesamtersparnis bei, während der Anteil aus der Verminderung des Stauaufkommens nur etwas mehr als $10 \%$ beträgt. Die Autoren weisen darauf hin, dass etwa Reboundeffekte durch eine erhöhte Nutzung von Fahrzeugen oder Beschäftigungs- und Einkommenseffekten in der Automobilwirtschaft oder der Verkehrsdienstleistungsbranche hier nicht berücksichtigt wurden dies müsse zukünftigen, umfangreich erweiterten Betrachtungen vorbehalten bleiben.

Eines der vorne erwähnten Defizite in der öffentlichen Debatte - die bisher zögerliche Auseinandersetzung insbesondere städtischer und regionaler (verkehrspolitischer) Akteure mit den möglichen Folgen der breiten Einführung automatisierten Fahrens in ihrem Verantwortungsbereich - greift ein Beitrag aus der Schweiz auf. Fabienne Perret, Remo Fischer und Holger Frantz haben im Rahmen einer interdisziplinären Studie in Zusammenarbeit mit zahlreichen schweizerischen Akteuren (u. a. Städte, Kantone, Transportunternehmen) die relevanten Aspekte des automatisierten Fahrens diskutiert und mögliche Auswirkungen abgeschätzt. Der gemeinsam festgelegte Fokus lag dabei auf planerischen Aspekten des Personenverkehrs auf Schiene und Straße. Sie führen aus, dass die beteiligten Städte und Regionen einen Teil der die öffentliche Diskussion prägenden Chancenerwartungen teilen: Pooling- und Sharing-Fahrzeuge führten zu Effizienz- und Flächengewinnen beim Parkraum, bei einer Re- bei überlagern sich mehrere Effekte: Mit einer (Voll-)Automatisierung wären Leerfahrten möglich (und je nach Servicekonzept wohl auch nötig), es entstehen neue Transportangebote, ohne die Notwendigkeit eines Fahrers mit klassischem Führerschein wird der Nutzerkreis des Individualverkehrs beträchtlich erweitert. Kapazitätserhöhungen infolge der Automatisierung könnten zusätzlichen Mehrverkehr mit sich bringen und zudem die Erreichbarkeiten so verändern, dass insbesondere ländliche Gemeinden und Agglomerationen profitieren, während Städte an Attraktivität einbüßen. Aufbauend auf diesen Befunden diskutieren die Autoren mögliche Maßnahmen bzw. Maßnahmenpakete, mittels derer Städte und Gemeinden lenkend eingreifen und mögliche Fehlentwicklungen korrigieren könnten.

Mit der heutigen Wahrnehmung des automatisierten Fahrens in der allgemeinen Öffentlichkeit und draus möglicherweise abzuleitenden Indizien für zukünftige Nachhaltigkeitseffekte setzt sich der Beitrag von Jürgen Hampel, Cordula Kropp und Michael Zwick auseinander. Im Rahmen der turnusmäßigen Repräsentativbefragung „TechnikRadar" haben sie einen thematischen Schwerpunkt auf das - in ihren Worten - ,voll autonome Fahren“ gelegt und rund 2.000 deutschsprachige Einwohner hinsichtlich ihrer Haltungen dazu interviewt. Ihre Ergebnisse vertiefen und bestätigen im Großen und Ganzen die Grundbeobachtungen älterer - teilweise mit anderen Stichprobendesigns und Formulierungen durchgeführter - Befragungen: Unter den Bürgerinnen und Bürgern herrschen erhebliche Zweifel an der Zuverlässigkeit und Sicherheit der Technik selbstfahrender Fahrzeuge, deutlich mehr als die Hälfte steht selbstfahrenden Fahrzeugen skeptisch gegenüber. Sorgen hinsichtlich Datensammlungen durch automatisierte Fahrzeuge (insbesondere, wenn diese keinen Bezug zum unmittelbaren Verkehrsmanagement haben), der Abgabe der Fahrverantwortung an automatisierte Systeme sowie

\section{Diskussionen zu den wissenschaftlich belegbaren Effekten des automatisierten Fahrens für ein nachhaltiges Mobilitätssystem stehen angesichts vieler Unsicherheiten erst am Anfang.}

duktion von Folgezeitlücken wären Kapazitätsgewinne und eine erhöhte Stabilität des Verkehrsflusses auf der Straße möglich. Durch das Vermeiden menschlicher Fehler könnte die Verkehrssicherheit erhöht werden, die technische Ausstattung der Fahrzeuge würde eine verbesserte Verkehrssteuerung ermöglichen. Mobility-as-a-Service-Angebote könnten die individuelle Mobilität erleichtern. Neue Angebote im ÖPNV und Sharing-Fahrzeuge verbesserten die Erschließungsqualität und ermöglichten Mobilität für bisher mobilitätseingeschränkte Nutzergruppen. Verbunden damit sind allerdings auch neue (bisher allerdings nur qualitativ beschreibbare) Herausforderungen. Im Zentrum stehen dabei die potenzielle Zunahme der verkehrlichen Nachfrage und die damit verbundene Erhöhung der Fahrleistung. Da-
Angst vor Hackerangriffen sind unter den wiederkehrenden Begründungen dafür. Aufbauend auf diesen Befunden diskutieren die Autorin und die Autoren einige Hypothesen zu möglichen Nachhaltigkeitseffekten im Rahmen des Drei-Dimensionen-Konzeptes.

Einer auf den ersten Blick in diesem Rahmen etwas ungewöhnlichen Technik widmen sich Michael Nentwich und Delila M. Horvath: einem drohnenbasierten Lieferverkehr. Absichtserklärungen von Unternehmen, einen solchen demnächst in größerem Umfang einführen zu wollen, finden sich seit einigen Jahren immer wieder in der öffentlichen Debatte. Die Autoren nähern sich diesen Ideen mit einer TA-Perspektive, skizzieren einen vorläufigen Analyserahmen und diskutieren erste 
Problemfelder für eine Gestaltung und Governance dieser Technik. Dabei sind einige Fragen durchaus denen ähnlich, die wir in einem Artikel in dieser Zeitschrift (Meyer-Soylu et al. 2014) hinsichtlich der Umsetzbarkeit eines individuellen Personenluftverkehrs in Städten aufgeworfen haben, andere sind eher güterverkehrsspezifisch. Insofern könnte es angeraten sein, Interdependenzen zwischen Lieferdrohnen und zukünftigen Personal Aerial Vehicles sowohl hinsichtlich der Möglichkeitsbedingungen als auch der Folgen zum Gegenstand einer zukünftigen TA-Studie zu machen und sich darüber hinaus auch der Frage zu widmen, ob und in welchem Umfang Automatisierung im Güterverteilverkehr in der Lage ist, individuellen Personenverkehr zu substituieren.

Einen Gastaufenthalt des Mobilitätsforschers Moshe Givoni von der Universität Tel-Aviv nutzten die Herausgeber für eine Diskussion zu den Entwicklungsperspektiven und möglichen Auswirkungen des automatisierten Fahrens. Das Gespräch wird in diesem Heft in der Rubrik Interview veröffentlicht. Auch wenn Givoni nicht ausschließt, dass automatisiertes Fahren zu nachhaltiger Mobilität beitragen könnte, befürchtet er, dass die Technologie autozentrierte Mobilitätmuster stärken und damit vorhandene Probleme eher intensivieren könnte. Viele positive Entwicklungen, wie der Trend zu mehr Fahrradverkehr in vielen Städten, könnten abgebremst werden. Die Thema-Herausgeber teilen Givonis These, dass nachhaltige Mobilität auch ohne automatisierte Autos vorstellbar sei und automatisiertes Fahren keineswegs automatisch zu mehr Nachhaltigkeit führen müsse. Torsten Fleischer hebt hervor, dass automatisiertes Fahren von recht unterschiedlichen Akteursgruppen vorangetrieben werde, dazu gehörten die Autoindustrie, aber als zweite Gruppe auch Plattformbetreiber - etwa Waymo (eine Tochter von Googles Muttergesellschaft Alphabet Inc.), Uber oder Baidu - sowie öffentliche Einrichtungen und Verkehrsunternehmen als dritte Gruppe. Entsprechend unterschiedlich seien die Vorstellungen über die zukünftige Rolle und Wirkungen von automatisiertem Fahren im Verkehrssystem. Jens Schippl weist darauf hin, dass die Autoindustrie selbst zwei unterschiedliche Visionen kommuniziert: die vom Wohlfühlort „eigenes Auto“, das zum Third Place neben Arbeitsort und Wohnort werde, sowie die von selbstfahrenden Taxis (Robo-Taxis), welche die Nutzung privater Pkws zurückdrängen würde. Klare Einigkeit bestand wiederum darin, dass sich angesichts der großen Veränderungspotenziale des automatisierten Fahrens die Entscheidungsträger noch deutlich mehr mit möglichen Entwicklungslinien und Folgen sowie vor allem mit politische Optionen, diese zu beeinflussen, auseinandersetzen sollten.

\section{Literatur}

acatech (Hg.) (2015): Neue autoMobilität. Automatisierter Straßenverkehr der Zukunft (acatech POSITION). München: Herbert Utz Verlag. Online verfügbar unter https://www.acatech.de/fileadmin/user_upload/ Baumstruktur_nach_Website/Acatech/root/de/Publikationen/ Stellungnahmen/NaM_acatech_POSITION_Neue_autoMobilitaet_Web.pdf, zuletzt geprüft am 11.06.2018.
BASt (Hg.) (2012): Rechtsfolgen zunehmender Fahrzeugautomatisierung. Gemeinsamer Schlussbericht der Projektgruppe. Berichte der Bundesanstalt für Straßenwesen. Fahrzeugtechnik Heft F 83. Bremerhaven: Verlag für neue Wissenschaft. Online verfügbar unter http://bast.opus.hbznrw.de/volltexte/2012/587/pdf/F83.pdf, zuletzt geprüft am 11. 06.2018.

CEC - Commission of the European Communities (2011): White paper. A roadmap to a single European transport area. Towards a competitive and resource efficient transport system. Luxembourg: Publications Office of the European Union. DOI: 10.2832/30955.

Meyer-Soylu, Sarah; Decker, Michael; Fleischer, Torsten; Schippl, Jens (2014): Zur Arbeit fliegen? Eine Technikfolgenabschätzung der Idee des individuellen Luftverkehrs für die Stadt. In: TATuP - Technikfolgenabschätzung in Theorie und Praxis 23 (1), S. 13-21.

SAE International (2016): Taxonomy and definitions for terms related to driving automation systems for On-Road motor vehicles. Warrendale, PA: SAE International.

Schippl, Jens; Fleischer, Torsten; Truffer, Bernhard (2018): Exploring potential impacts of societal dynamics on the development of autonomous cars. Proceedings of the $7^{\text {th }}$ Transport Research Arena TRA 2018, April 16-19, 2018, Vienna, Austria. Im Erscheinen.

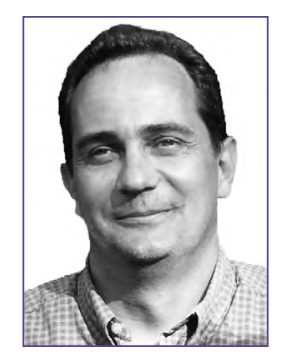

\section{TORSTEN FLEISCHER}

hat sich nach einem Physikstudium für die Forschung und Politikberatung zu Prozessen technischen Wandels und deren Wechselwirkung mit gesellschaftlichen Veränderungen entschieden. Er ist heute Leiter des Forschungsbereichs „Innovationsprozesse und Technikfolgen " am Institut für Technikfolgenabschätzung und Systemanalyse (ITAS) des KIT. Im Rahmen mehrerer dort angesiedelter Forschungsprojekte beschäftigt er sich aktuell auch mit den Möglichkeitsbedingungen und Folgen automatisierten Fahrens.

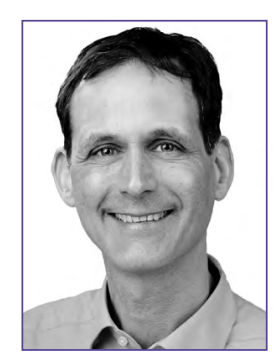

\section{JENS SCHIPPL}

hat Geographie, Biologie und Soziologie studiert. Seit über 15 Jahren arbeitet er im Feld der Technikfolgenabschätzung, seit 2006 am Institut für Technikfolgenabschätzung und Systemanalyse (ITAS) des KIT. Zu seinen Forschungsschwerpunkten gehören die Themen soziotechnischer Wandel, Foresight und Technikfolgenabschätzung in den Bereichen Mobilität und Energie. Mögliche Entwicklungspfade und Wirkungen einer fortschreitenden Digitalisierung im Mobilitätssystem stehen dabei im Mittelpunkt. 


\title{
Ist automatisiertes Fahren nachhaltig?
}

\author{
Entwicklungstendenzen und Forschungsüberlegungen \\ angesichts geringer Akzeptanz
}

Karsten Weber, Institut für Sozialforschung und Technikfolgenabschätzung, Ostbayerische Technische Hochschule Regensburg, Galgenbergstraße 24, 93053 Regensburg (Karsten.Weber@oth-regensburg.de), (1) orcid.org/0000-0001-8875-2386

Sonja Haug, Institut für Sozialforschung und Technikfolgenabschätzung, Ostbayerische Technische Hochschule Regensburg (Sonja.Haug@oth-regensburg.de)

Automatisiertes Fahren stößt derzeit noch auf große Skepsis. Eine disruptive Strategie bei der Einführung (voll-)automatisierten Fahrens könnte daher auf fehlende Akzeptanz treffen. Um dem zu entgehen, laufen evolutionäre Strategien darauf hinaus, durch die Entwicklung adaptiver Fahrassistenzsysteme Vertrautheit, Vertrauen und damit Akzeptanz bei den prospektiven NutzerInnen zu schaffen. Erste Ergebnisse einer Pilotstudie lassen jedoch Zweifel an der Nachhaltigkeit dieser Strategie aufkommen.

\section{Is automated driving sustainable?}

Development trends and research considerations in view of

low acceptance rates

Automated driving is currently viewed with great skepticism. A disruptive strategy for the introduction of (fully) automated driving could therefore be met with a lack of acceptance. To avoid this, evolutionary strategies aim to create familiarity, trust, and thus acceptance among the prospective users through the development of adaptive driver assistance systems. However, the preliminary results of a pilot study raise doubts about the sustainability of this strategy.

Keywords: driver assistance systems, automated driving, acceptance, sustainability

\section{Einleitung}

Verschiedene aktuelle Studien (z. B. Bertelsmann 2017; Ernst \& Young 2017; Deloitte 2017) weisen auf, dass vollautomatisiertes Fahren auf erhebliche Skepsis stößt: Bis zu zwei Drittel der in den Studien Befragten äußern Ablehnung in Hinblick auf vollautomatisierte Pkw, oft mit dem Hinweis auf die Unsicherheit der Technik. Die fehlende Akzeptanz erklärt sich vermutlich u. a. durch Berichte über Unfälle mit vermeintlich vollautomatisierten Pkw, doch könnte deren Wurzel auch in der Angst vor Kontrollverlust und grundsätzlichem Misstrauen gegenüber einer weitgehend unbekannten Technik liegen, mit der außerhalb von Testzentren kaum jemand umfassende Erfahrung hat. Akzeptanzmindernd könnte zudem sein, dass vollautomatisiertes Fahren es erschweren bis verunmöglichen würde, einem eigenen Fahrstil Ausdruck zu verleihen. Der Untersuchung dieser Vermutung ist der vorliegende Beitrag gewidmet.

Firmen, die bisher nicht als Automobilbauer aufgetreten sind - z. B. Uber, Apple oder Google -, scheinen einen disruptiven Ansatz zu verfolgen und möglichst viele Zwischenphasen zum vollautomatisierten Fahren überspringen zu wollen, um sofort mit führerlosen Fahrzeugen neue Geschäftsmodelle anbieten zu können. „Disruptiv“ bezieht sich dabei nicht auf die verbaute Fahrzeugtechnik (Sensoren, Aktoren, Steuerungsrechner etc.), sondern in erster Linie auf die Möglichkeit bzw. Unmöglichkeit auf das Fahrzeugverhalten einwirken zu können. Ein fahrerloser Pkw oder Lkw bedeutet einen radikalen Bruch mit rund 120 Jahren Automobilgeschichte und einer noch längeren Geschichte menschlicher Mobilität. Die Disruption findet also aus Perspektive der FahrzeugnutzerInnen und anderer VerkehrsteilnehmerInnen statt.

Andere Unternehmen, z. B. die großen deutschen Automobilhersteller, setzen auf eine evolutionäre Entwicklung mit einer schrittweisen und jeweils partiellen Übernahme von Steuerungsaufgaben durch Fahrassistenzsysteme, was den FahrzeugnutzerInnen ebenso wie anderen VerkehrsteilnehmerInnen die Chance
This is an article distributed under the terms of the Creative Commons Attribution License CCBY 4.0 (https://creativecommons.org/licenses/by/4.0/)

https://doi.org/10.14512/tatup.27.2.16

Submitted: 23. 02.2018. Peer reviewed. Accepted: 11. 05.2018 
einer langsamen Gewöhnung an die für sie neue Technik eröffnet. Als Konsequenz daraus würden noch über lange Zeit hinweg sowohl rein von Menschen gesteuerte, teilassistierte als auch (irgendwann) hoch- und vollautomatisierte Fahrzeuge gleichzeitig auf den Straßen unterwegs sein. Die gültige Rechtslage scheint diese Strategie zu bestätigen, denn vorerst wird die Haftung bei den FahrzeughalterInnen verbleiben - was aber den Wunsch nach Kontrolle über das Fahrzeug aufseiten der FahrerInnen verstärken und die Akzeptanz vollautomatisierten Fahrens verringern könnte.

Obwohl vermutet werden kann, dass bei der Wahl einer evolutionären Entwicklung nicht zuletzt Marktüberlegungen und damit ökonomische Faktoren eine wichtige Rolle spielen, kann sie auch dazu geeignet sein, Ängste aufseiten der Öffentlichkeit abzubauen sowie die Akzeptanz für Fahrassistenzsysteme und, auf längere Sicht, für automatisiertes Fahren der drei höchsten Stufen (hochautomatisiert, vollautomatisiert, fahrerlos) bei den prospektiven FahrerInnen zu erhöhen. Im Folgenden soll daher etwas ausführlicher auf diese evolutionäre Strategie eingegangen werden, da sie die Motivation der später beschriebenen Pilotstudie liefert. Mit dieser Studie sollten Grundlagen zur fahrerbasierten Parametrisierung von Fahrassistenzsystemen erarbeitet werden, doch die Ergebnisse lassen zusätzlich Schlüsse in Bezug auf die Frage zu, ob parametrisierte bzw. adaptive Fahrassistenzsysteme Nachhaltigkeitsgewinne im Sinne einer Kraftstoffeinsparung und eines risikoloseren Fahrverhaltens erzielen könnten. Basierend auf dem Drei-Säulen-Modell der Nachhaltigkeit mit den Komponenten Ökologie, Ökonomie und Soziales (Enquete-Kommission 1998) bezieht sich der Beitrag in erster Linie auf die ökologischen und sozialen Aspekte. Der zweite Teil des folgenden Textes besteht aus der Beschreibung der Pilotstudie und deren Zielsetzung sowie aus einigen Schlussfolgerungen, die sich aus den Ergebnissen ziehen lassen.

Es muss bereits an dieser Stelle betont werden, dass es sich allenfalls um Aussagen in der Tendenz handelt, da die empirische Basis der Studie zu gering ist, um verlässlichere Aussagen treffen zu können. Zudem kann man aus methodischer Sicht hinterfragen, inwieweit das induktive statistische Verfahren der Clusterbildung sowie die interpretierende Bewertung der Fahrleistungen belastbare Aussagen zulassen. Ungeachtet dessen halten wir die Schlussfolgerung für richtig, dass die Parametrisierung von Fahrassistenzsystemen anhand von Fahrtypen zur Erhöhung der Akzeptanz in Hinblick auf die beschriebenen Effizienzgewinne kontraproduktiv ist.

\section{(Weiter-)Entwicklung adaptiver Fahr- assistenzsysteme und deren Akzeptanz}

Eine Motivation für die weiter unten vorgestellte Pilotstudie liegt darin, dass heute bereits verbaute Fahrassistenzsysteme nicht immer genutzt werden (Reagan at al. 2018). Aus Sicht der Automobilhersteller stellt diese Verhaltensweise zunächst ein betriebswirtschaftliches und Marketingproblem dar, volkswirt- schaftlich bedeutet es Ressourcenverschwendung und in Hinblick auf Verkehrssicherheit folgt aus der Nichtnutzung von Fahrassistenzsystemen in vielen Fällen, dass das Potenzial für Gewinne im Bereich der Verkehrssicherheit und vielleicht auch der Ressourceneffizienz nicht voll ausgeschöpft wird.

Wie oben bereits angedeutet, stoßen Fahrassistenzsysteme bzw. das teilassistierte ebenso wie das hoch- und vollautomatisierte Fahren immer noch auf erhebliche Skepsis, wobei entsprechende Studien stark länderabhängige und teilweise auch sehr unterschiedliche Ergebnisse zeigen. Das mag u. a. daran liegen, dass im Gegensatz zur etablierten Forschung bzgl. der Akzeptanz technischer Innovationen und technikgestützter Dienstleistungen im Allgemeinen die Forschung zur Kundenwahrnehmung noch in einem frühen Stadium steckt (Woisetschläger 2015). Existierende Studien und Projekte, die nicht zuletzt die individuelle und gesellschaftliche Akzeptanz von Fahrassistenzsystemen in den Blick nehmen, sind z. B.:

- Das seit einigen Jahren laufende Förderprojekt Villa Ladenburg (Fraedrich und Lenz 2014) hat bereits eine Reihe von Publikationen nach sich gezogen, in denen z. B. Entwicklungstrends, Veränderungen der Mobilität durch autonome Fahrzeuge, Geschäftsmodelle und Akzeptanzfragen behandelt werden.

- Arndt (2011) hat ein Modell zur Vorhersage und Analyse der Akzeptanz von Fahrassistenzsystemen entwickelt, um Entscheidungen beim Kauf von Pkw beschreiben und prognostizieren zu können.

- Payre et al. (2014) schlagen in eine ähnliche Kerbe wie Arndt (2011) und untersuchen Einstellungen zu und grundsätzliche Akzeptanz von autonomen Fahrzeugen.

- Verberne et al. (2012) sehen Vertrauen in Fahrassistenzsysteme als wesentlichen Akzeptanzfaktor. Damit FahrerInnen die jeweilige Verkehrssituation besser einschätzen können, obwohl sie nicht (völlig) die Kontrolle über das Fahrzeug besitzen, wird der fahrzeugübergreifende Austausch von Informationen zwischen den Fahrassistenzsystemen vorgeschlagen.

Existierende Fahrassistenzsysteme sind in vielen Ländern bereits wichtiger Bestandteil moderner Fahrzeuge, die FahrerInnen vorrangig bei der Fahrt unterstützen und zeitgleich den Komfort steigern sowie die Unfallgefahr minimieren sollen. Die gerade genannten und vermutlich auch viele andere Studien legen allerdings solche Fahrassistenzsysteme zugrunde, die nicht oder nur in geringem Maße adaptiv und parametrisierbar gestaltet sind. Das Fahrzeugverhalten wird durch die ab Werk vorgegebene Programmierung der Systeme bestimmt und kann von den FahrerInnen kaum oder gar nicht beeinflusst werden.

Um die in den Studien teilweise schlecht ausfallende Akzeptanz und damit den Nutzungsgrad zu steigern sowie ihre Anpassungsfähigkeit an die gegebene Verkehrssituation zu erhöhen wird nun seit einigen Jahren versucht, Fahrassistenzsysteme an die jeweils gegebenen Umweltbedingungen und/oder die Charakteristika der FahrerInnen anzupassen. Beispiele sind 
die Arbeiten von Wang et al. (2014), die Geschwindigkeit und Abstand zum vorausfahrenden Fahrzeug an die individuelle Gasund Bremspedalbedienung anpassen möchten und damit ein erweitertes Adaptive Cruise Control-System (ACC) entwickeln wollen. Dannheim et al. (2013) gehen darüber hinaus und integrieren Wetterinformationen in ein ACC, um ein Intelligent Speed Adaptation-System (ISA) zu realisieren.

Eine Adaption des Fahrstils im Rahmen einer evolutionären Weiterentwicklung existierender Fahrassistenzsysteme könnte auch auf kategorisierten Fahrstilen oder -typen beruhen (Haböck et al. 2016). Die in diesem Bereich durchgeführten Studien befassen sich z. B. mit den Motivationen und Interessens-
Langzeitprofil einer typischen Fahrweise führt. Es wäre so denkbar, dass Fahrassistenzsysteme Fahrstile aus dem tatsächlichen Fahrverhalten lernen, doch dies hätte den Nachteil, dass dabei potenziell auch Fehlverhalten im Hinblick auf die Zielgröße kraftstoffsparendes Fahren gelernt werden könnte. Auf diesen Aspekt wird am Ende des Textes zurückzukommen sein.

Will man Fahrstile zur Auswahl stellen oder das Assistenzsystem an das bisherige Fahrverhalten adaptieren, müssen die Charakteristiken der Fahrstile zunächst vordefiniert werden. Hierzu grundlegende Methoden zu entwickeln und erste Ergebnisse zu erzielen, war Ziel einer Pilotstudie mit explorativem Charakter (Haug et al. 2017), die 2015 und 2016 am Institut für

\section{Sollen Nachhaltigkeitsgewinne erzielt werden, müssten}

\section{Fahrassistenzsysteme ein nachhaltiges Fahrzeugverhalten implementieren statt menschliche Fahrtypen zu kopieren.}

schwerpunkten der FahrerInnen, um Rückschlüsse auf Fahrtypen treffen zu können. So wurden z. B. im Rahmen der Studie „Fernfahrer 2.0: Der Mensch im Transport- und Logistikmarkt" auf Basis von Experteninterviews vier Typen entwickelt: $\operatorname{der} B e-$ rufene, der Überzeugte, der Rationale und der Zufällige (Lohre et al. 2014). Das SANTOS-Projekt (situationsangepasste und Nutzer-Typ-zentrierte Optimierung von Systemen zur Fahrerunterstützung) verfolgt das Ziel, die aktive Sicherheit durch eine situationsangepasste und nutzerorientierte Gestaltung der Assistenz- und Kommunikationsfunktionen im System Fahrer-Fahrzeug-Umgebung zu erhöhen (König et al. 2002). Hier wurden die ProbandInnen, die in einem Demonstrator einen Fahrtest absolvierten, in fünf Fahrtypen eingeteilt: sportlich-draufgängerisch, dynamisch-progressiv, routiniert-abgeklärt, unauffällig-konservativ sowie ängstlich-zurückhaltend.

\section{Pilotstudie}

\section{Zielsetzung}

Eine teilautomatisierte Regelung eines Fahrassistenzsystems kann mit sich bringen, dass FahrerInnen das Gefühl der eigenen Kontrolle verlieren und befürchten, Fahrgewohnheiten aufgeben zu müssen. Hier setzt der Ansatz der Adaption des Fahrassistenzsystems an (Haug et al. 2017). Eine bereits in verschiedenen Fahrzeugen gängige Möglichkeit besteht darin, dass die FahrerIn selbst einen Fahrstil aus einem gegebenen Angebot auswählt. Dies könnte z. B. ein energiesparender Fahrmodus („Eco“) oder ein dynamischer Fahrmodus (,Sportlich“) sein. Vor Beginn einer Fahrt wird dann die FahrerIn automatisch erkannt und die Fahrassistenzsysteme dem entsprechenden Fahrstil passend parametrisiert. Ein adaptives System beruht hingegen auf der kontinuierlichen Beobachtung des Fahrverhaltens, die zu einem
Sozialforschung und Technikfolgenabschätzung (IST) der Ostbayerischen Technischen Hochschule (OTH) Regensburg in $\mathrm{Zu}-$ sammenarbeit mit der Bertrandt $\mathrm{GmbH}$ durchgeführt wurde. Im Rahmen der Studie wurde das Fahrverhalten unterschiedlicher Personengruppen vergleichend untersucht und auf dieser Grundlage Typisierungen vorgenommen.

\section{Forschungsdesign}

Die Methodik stellt eine Kombination und Fortentwicklung bestehender Ansätze dar. Um die Komplexität der Fahrsituation aus verschiedenen Perspektiven erfassen zu können, orientierte sich das Forschungsdesign am Ansatz der Triangulation (Flick 2016), d. h. es beinhaltete mit quantitativen (Fragebogen) wie auch qualitativen Methoden (teilnehmende Beobachtungen, narrative Leitfadeninterviews) mehrere sich ergänzende und gegenseitig korrigierende Methoden. Zur Rekonstruktion der Fahrsituation waren im Testfahrzeug zwei USB-Kameras angebracht, die in Fahrtrichtung filmten sowie die Fahrerin/den Fahrer während der Fahrt aufnahmen. Das Testfahrzeug (Audi A7 mit Automatikgetriebe) war mit diversen computergestützten Messvorrichtungen ausgestattet, welche die technischen Fahrdaten aufzeichneten (Haug et al. 2017). Da für die Studie das Fahrverhalten der ProbandInnen ohne Unterstützung von Fahrassistenzsystemen untersucht werden sollte, wurden vor Beginn der Testfahrten alle integrierten Fahrassistenzsysteme (Abstandsregeltempomat, Spurhalteassistent) deaktiviert.

Damit das Fahrverhalten der ProbandInnen in möglichst vielen unterschiedlichen Szenarien getestet werden konnte, setzte sich die Teststrecke aus den Teilstreckenbereichen Stadtverkehr, Landstraße und Autobahn zusammen. Entsprechend der Angaben des Kartendienstes Google Maps betrug der gesamte Streckenverlauf 55,2 km bei einer durchschnittlichen Fahrtzeit von 1:22 Stunden. Alle teilnehmenden Personen fuhren die gleiche 
Strecke; dabei wurde darauf geachtet, dass sich die Fahrbedingungen möglichst glichen. Die Testfahrten wurden im Herbst durchgeführt.

Die ProbandInnenauswahl erfolgte entsprechend des Ansatzes des Theoretical Samplings mittels einer bewussten Zusammenstellung der Stichprobe durch zuvor festgelegte und für das Forschungsvorhaben relevante Merkmale sowie deren minimale und maximale Kontrastierung. Im Rahmen der Untersuchung unterschiedlicher Fahrstile ergaben sich für den Stichprobenplan folgende Merkmale mit ihren Ausprägungen: Alter (junges Alter [18-25], mittleres Alter [26-55] und gehobenes Alter [ab 56]), Geschlecht (weiblich, männlich) sowie Fahrpraxis (wenig, mittel und viel fahrend), wobei sich die Fahrpraxis aus den jährlich gefahrenen Kilometern und der wöchentlichen Nutzung ergab. Insgesamt wurden 18 ProbandInnen ausgewählt, so dass jede Merkmalskombination vertreten war.

\section{Erhebungsinstrumente}

Die Studie beinhaltete eine Reihe unterschiedlicher Erhebungsinstrumente:

- Die schriftliche Fragebogenerhebung erfolgte kurz vor der Testfahrt. Der standardisierte Fragebogen (Schnell et al. 2013) beinhaltete neben Fragen zu persönlichen soziodemografischen Angaben (Geschlecht, Alter, Dauer des Führerscheinbesitzes, Angaben zum aktuellen persönlichen Empfinden) auch Fragen mit größtenteils 5-stufigen Skalen zur persönlichen Fahrpraxis (durchschnittliche Fahrleistung im Jahr in Kilometer, Angaben zum eigenen bzw. üblicherweise genutzten Pkw, Angaben zur Erfahrung mit Automatikschaltgetrieben und Fahrassistenzsystemen, wöchentliche Fahrtätigkeit, Angaben zur Pkw-Nutzung, zum hauptsächlich gefahrenen Strecken- und Straßentyp) und Fragen zum persönlichen Fahrverhalten (Einstellung zum Autofahren, Angaben zum Verhalten bei Mitfahrern und im allgemeinen Straßenverkehr, angelehnt an König et al. 2002).

- Während der Fahrt wurden folgende Signalmessungen und Kameraaufnahmen automatisiert vorgenommen: Aufnahme der Bussignale (CAN, Flexray), USB-Kamera 1: Aufnahme des Fahrzeuginnenraums, USB-Kamera 2: Aufnahme der vorausliegenden Strecke.

- Die offene teilnehmende Beobachtung während der Fahrt erfolgte durch zwei anwesende BeobachterInnen. Die Anwesenheit von zwei BeobachterInnen (Forscher-Triangulation, vgl. Flick 2016) besitzt den Vorteil, dass einerseits die subjektive Verzerrung der Beobachtung minimiert und andererseits die ProbandInnen durch die/den zweiten BeobachterIn in Gespräche verwickelt und so von der künstlich geschaffenen Beobachtungssituation abgelenkt werden konnten.

- Während der Fahrt wurde ein Beobachtungsprotokoll mit folgendem Inhalt angefertigt: Situationsbeschreibung (Datum, Uhrzeit, Aussagen zum Wetter, Zustand der ProbandInnen vor und während der Fahrt, Anwesende und Besonderheiten), tabellarischer Streckenverlauf (Unterteilung der Teststrecke in kleinere Abschnitte zur besseren Mitschrift der Beobachtungen während der Fahrt), Notizbereich (Platz für gesonderte Notizen) und Einschätzung der FahrerInnenleistung hinsichtlich Sportlichkeit, Verkehrssicherheit und Effizienz in den drei Teilstreckenbereichen Stadt, Land und Autobahn.

- Zusätzlich wurden die Videoaufzeichnungen der Fahrten durch eine/n dritten BeobachterIn gesichtet und ausgewertet.

- Bei dem abschließenden Reflexionsgespräch mit den ProbandInnen über die kurz zuvor erfolgte Testfahrt handelte es sich um eine mündliche teilstrukturierte und leitfadengestützte Befragung.

\section{Auswertung}

Auf Basis des Ratingverfahrens (Pauli 2014) erfolgte im Anschluss an die Fahrt bzw. Sichtung der Videoaufzeichnung eine jeweils durch die drei BeobachterInnen vorgenommene und voneinander unabhängige Bewertung der Fahrleistung der ProbandInnen für die Teilstreckenbereiche Stadt, Land und Autobahn hinsichtlich Verkehrssicherheit, Sportlichkeit und Effizienz. Das Ratingverfahren ist eine Methode, mittels derer die komplexen Merkmale einer Beobachtungssituation bzw. derer jeweiligen Ausprägung eingeschätzt und bewertet werden. Dabei stützt sich das Beobachtungsverfahren auf die Urteilsfähigkeit der BeobachterInnen, welche den Gesamteindruck adäquat widerspiegeln soll (Pauli 2014). Die Fahrleistung der ProbandInnen hinsichtlich Verkehrssicherheit, Sportlichkeit und Effizienz wurde anhand einer 5-stufigen Ratingskala beurteilt, wobei 1 die minimale Ausprägung und 5 die maximale Ausprägung der Fahreigenschaft angibt.

Anhand der Beobachtungsprotokolle wurde jeder Indikator einzeln und unabhängig nach der Methode des Ratingverfahrens von den BeobachterInnen zwischen 1 (minimale Ausprägung des Indikators) und 5 (maximale Ausprägung des Indikators) bewertet.

- Verkehrssicherheit wird durch die Variablen Regelkonformes Verhalten im Straßenverkehr und Aufmerksamkeit sowie die Indikatoren Größe des Abstands (Stadtverkehr, Landstraße, Autobahn), Intensität der Verkehrsbeobachtung, Höhe der Geschwindigkeit (entsprechend StVO), Intensität der vorausschauenden Fahrweise, Intensität der Beachtung der Vorfahrtsregeln, Regelkonformes Verhalten bei der Autobahnauffahrt, Ampelverhalten und Art des Kurvenschneidens beschrieben.

- Sportlichkeit bezeichnet eine sportliche Fahrweise, die sich vor allem in den Variablen Spurführung, Geschwindigkeit und Beschleunigungsverhalten sowie deren Indikatoren Anzahl und Art des Kurvenschneidens, Höhe der Geschwindigkeit auf gerader Strecke (Landstraße und Autobahn), Anzahl und Dauer der Längsbeschleunigung und Kickdowns sowie Anzahl und Art des Bremsverhaltens widerspiegelt.

- Effizienz bezeichnet eine kraftstoffsparende und energieeffiziente Fahrweise, die sich aus den Variablen Geschwindigkeit, Beschleunigung und Vorausschauende Fahrweise mit 
den dazugehörigen Indikatoren Art der Kurvendurchfahrt (Fahrpedal- und Bremstätigkeit), Höhe der Geschwindigkeit auf gerader Strecke (Landstraße und Autobahn), Vergleich zwischen Gas- und Bremsverhalten, Intensität der Längsbeschleunigung sowie Größe des Abstands (Stadtverkehr, Landstraße, Autobahn) ergibt.

Die statistische Auswertung erfolgte mittels Clusteranalyse (Backhaus et al. 2015), einer Methode zur Klassifikation von Personen und ihrer Merkmale. Bei dem verwendeten $k$-Means-Clusterverfahren bildet ein Algorithmus aus den vorgegebenen Daten eine Anzahl an Gruppen, bei denen die Summe der quadrierten Abweichungen von den Cluster-Schwerpunkten minimal ist.

Die Auswertung ergab die drei Fahrtypen Ausgeglichen, Sicher und Effizient sowie Sportlich, die mittels der Variablen der Fremdeinschätzung (Mittelwerte der Indikatoren-Bewertungen für Verkehrssicherheit, Sportlichkeit und Effizienz) und deren Differenzen gebildet wurden. Die Gruppenunterschiede sind signifikant (getestet mittels einfaktorieller ANOVA).

\section{Ergebnis}

- Die Gruppe der Ausgeglichenen kennzeichnet einen annähernd gleich hohen Mittelwert für Sicherheit, Sportlichkeit und Effizienz. ProbandInnen, die diesem Fahrtypus zugeordnet wurden, wiesen eine sowohl verkehrssichere, als auch sportliche und effiziente Fahrweise auf. 10 der 18 Probanden konnten diesem Fahrtypus zugeordnet werden (56\%).

- Die Gruppe Sicher und Effizient ist dadurch gekennzeichnet, dass die Mittelwerte für Sicherheit und Effizienz im Vergleich zu dem Wert für Sportlichkeit höher sind. ProbandInnen, die diesem Fahrtypus zugeordnet wurden, kennzeichnet eine eher verkehrssichere und effiziente als eine sportliche Fahrweise. Diesem Fahrtypus entsprechen 5 von 18 Probanden $(28 \%)$.

- Die ProbandInnen, die dem Fahrtypus Sportlich zugeordnet werden konnten, haben einen höheren Wert bei Sportlichkeit als bei Sicherheit oder Effizienz. 11\% der ProbandInnen wurden diesem Typus zugeordnet.

Bei keinem der drei Fahrtypen konnte ein signifikanter Zusammenhang zu den demografischen Angaben der ProbandInnen (Geschlecht, Alter) oder Angaben zur Fahrpraxis und Fahrleistung festgestellt werden; ein Rückschluss zum Beispiel vom Geschlecht, dem Alter oder der Fahrleistung auf den jeweiligen Fahrstil ist also nicht möglich.

Wie schon bemerkt wurden Sportlichkeit, Sicherheit und Effizienz auf einer Skala von 1 (minimale Ausprägung) bis 5 (maximale Ausprägung) durch die BeobachterInnen bewertet (Fremdeinschätzung). Sowohl im Fragebogen vor der Testfahrt als auch in der Befragung nach der Testfahrt wurden die ProbandInnen gebeten, sich hinsichtlich ihrer Fahrweise selbst einzuschätzen. Zwischen Selbsteinschätzung (Interview) und Fremdeinschätzung konnte kein signifikanter Zusammenhang festge- stellt werden. Gründe hierfür könnten zum einen sein, dass die Selbsteinschätzung nicht valide ist und zum anderen, dass die Definitionen und Vorstellungen einer verkehrssicheren, sportlichen und effizienten Fahrweise zwischen ProbandInnen und BeobachterInnen stark voneinander abweichen.

Der Vergleich der Mittelwerte der Selbst- und Fremdwahrnehmung bzgl. der Einschätzung zur Sicherheit ergibt hierbei, dass sich alle drei Fahrtypen überschätzen, wobei der Fahrtypus Sicher und Effizient sich nur gering überschätzt, der Fahrtypus Ausgeglichen etwas mehr und die Sportlichen am meisten. Bezüglich der Einschätzung der ProbandInnen zur Sportlichkeit im Vergleich mit den Bewertungen der BeobachterInnen schätzt sich der Fahrtypus Ausgeglichen sehr gut ein und unterschätzt sich nur wenig, der Fahrtypus Sicher und Effizient überschätzt sich hingegen und die Sportlichen unterschätzen ihre Fahrleistung hinsichtlich Sportlichkeit sehr. Der Vergleich der Selbstund Fremdwahrnehmung zur Effizienz zeigt, dass der Fahrtypus Sicher und Effizient sich unterschätzt und die Fahrtypen Ausgeglichen und Sportlich sich hinsichtlich ihrer effizienten Fahrweise überschätzen. Mit anderen Worten: Zwei Drittel der ProbandInnen fahren weniger kraftstoffsparend als sie annehmen. Insbesondere dieses Ergebnis muss für die Schlussfolgerungen bzgl. der Unterstützung von Nachhaltigkeit durch Fahrassistenzsysteme beachtet werden.

Wie ein direkter Vergleich der drei Fahrtypen zeigt, weicht der Fahrtypus Sportlich im Vergleich zu den Fahrtypen Sicher und Effizient sowie Ausgeglichen bei allen drei Indikatoren am meisten mit seiner Selbstwahrnehmung von der Fremdeinschätzung ab.

\section{Diskussion}

Da die Untersuchung als eine Art Vorstudie konzipiert war, um mögliche Hinweise auf unterschiedliche Fahrtypen entdecken zu können, ist die Stichprobe sehr klein gehalten. In einer Folgestudie müsste daher die Stichprobe vergrößert werden. Auch ließ sich nicht vermeiden, dass sich die Ausgangsbedingungen bei den Fahrten unterschieden (Wochentag, Uhrzeit, Wetterbedingungen).

Wie die Studie gezeigt hat, kann der Fahrstil anhand der drei Eigenschaften Verkehrssicherheit, Sportlichkeit und Effizienz sowie deren Indikatoren beschrieben werden. Auch wenn die Stichprobengröße sehr gering ist, konnten mittels der Bewertung des Fahrstils der ProbandInnen durch drei BeobachterInnen während der Testfahrten drei signifikant unterschiedliche Fahrtypen definiert werden. Weniger als ein Drittel kann dem ressourcenschonenden Fahrtypus der Sicheren und Effizienten zugeordnet werden.

Weitaus schwerwiegender hingegen scheint, dass zwischen Fremdeinschätzung (Beobachtung) und Selbsteinschätzung kein signifikanter Zusammenhang besteht und Differenzen bei allen Fahrtypen auftraten. Im Durchschnitt unterschätzen sich die Probanden hinsichtlich ihrer sportlichen Fahrweise. In Bezug auf Sicherheit und Effizienz bewerten sich die Befragten jedoch generell selbst besser als die BeobachterInnen; dies gilt in be- 
sonderem Maße für den Fahrtypus der Sportlichen. In Punkto vorausschauende, kraftstoffsparende und energieeffiziente Fahrweise ist Selbstüberschätzung somit weit verbreitet.

\section{Schlussfolgerungen: Adaptiv ja, aber nachhaltig?}

Ob die beschriebene Strategie einer evolutionären Entwicklung von Fahrassistenzsystemen zu einer ökonomisch, ökologisch und sozial nachhaltigen Mobilität beitragen kann, ist mit dieser Pilotstudie nicht endgültig beantwortet, doch lassen die Ergebnisse erhebliche Zweifel zumindest in Bezug auf die ökologische (Kraftstoffverbrauch und in der Folge Schadstoffausstoß) und die soziale (Verkehrssicherheit) Nachhaltigkeitsdimension aufkommen. Betriebswirtschaftlich macht es vermutlich Sinn, Fahrassistenzsysteme schrittweise einzuführen, weil so z. B. Investitionen über längere Zeiträume verteilt und Argumente für neue Fahrzeuggenerationen abgeleitet werden können. $\mathrm{Ob}$ die schrittweise Einführung jedoch volkswirtschaftlich Sinn ergibt und damit die ökonomische Dimension der Nachhaltigkeit adressiert wird, sei dahingestellt - womöglich würden öffentlich geförderte Infrastrukturen aufgebaut, die schon nach eher kurzer Zeit obsolet werden. Das muss hier jedoch eine bloße Vermutung bleiben.

Zieht man nun die oben skizzierten Ergebnisse in Betracht und würden auf Basis entsprechend breit angelegter Studien, die z. B. auf den hier präsentierten Verfahren und Ergebnissen aufbauten, Fahrtypen definiert und programmiert, die die FahrerInnen in Zukunft auswählen könnten, wären die so parametrisierten Fahrassistenzsysteme weniger ressourcensparend und unsicherer als sie durch ihre Benennung signalisierten. Mögliche Effizienzgewinne in Gestalt eines geringeren Kraftstoffverbrauchs oder erhöhter Sicherheit wären mit ihnen also gerade nicht zu erzielen. Dies ist darin begründet, dass die Fahrstile die Fehleinschätzungen der untersuchten FahrerInnen beinhalteten. Denkbar wäre natürlich, dass bei systematischen Verzerrungen diese herausrechenbar wären und so die Fehleinschätzungen nicht in die Parametrisierung der Fahrstile einflössen dann aber spiegelten diese nicht mehr den erwarteten Fahrstil wider und könnten somit auf mangelnde Akzeptanz treffen. Sinn und Zweck parametrisierter Fahrassistenzsysteme in Hinblick auf Akzeptanzsteigerung würden somit verfehlt.

Eine Alternative wäre, Fahrassistenzsysteme nicht ab Werk zu parametrisieren, sondern selbst den Fahrstil der NutzerInnen eines Fahrzeugs erlernen zu lassen, um danach einen der jeweiligen Person angemessenen Fahrstil zeigen zu können. Nach den oben geschilderten Erfahrungen könnte das aber wiederum bedeuten, dass die Fahrzeuge ein Fahrverhalten lernten, das in Hinsicht auf Sicherheit und Kraftstoffverbrauch nicht optimal wäre. Kurzum: Parametrisierte wie adaptive Fahrassistenzsysteme, die dazu dienen sollen, durch eine angepasste assistierte Fahrweise die Attraktivität und Akzeptanz des teilassistierten, hochautomatisierten und irgendwann vollautomatisierten Fahrens zu er- höhen, würden die Argumente, die objektiv für die Nutzung von Fahrassistenzsystemen sprechen, (zumindest teilweise) unterlaufen (vgl. Hartwich et al. 2018).

In Bezug auf die Transformation des bundesdeutschen Verkehrssystems hin zu größerer Nachhaltigkeit ist angesichts dieser Erkenntnisse eher Skepsis angebracht. Sollen Nachhaltigkeitsgewinne - also geringerer Kraftstoffverbrauch und erhöhte Sicherheit - erzielt werden, müssten Fahrassistenzsysteme das Fahrzeugverhalten, der jeweiligen Automatisierungsstufe entsprechend, möglichst vollständig bestimmen und dabei in erster Linie auf ein nachhaltiges Fahrzeugverhalten statt auf die Kopie menschlicher Fahrtypen hin ausgerichtet sein. Das bedeutete notwendigerweise einen weitreichenden Kontrollverlust aufseiten der FahrerInnen. Damit wäre jedoch nicht nur marketingtechnisch eine erhebliche Herausforderung für die Fahrzeughersteller verbunden, sondern dies berührte gerade in Deutschland gesellschaftlich tief verankerte Vorstellungen von Freiheit.

\section{Literatur}

Arndt, Stephanie (2011): Evaluierung der Akzeptanz von Fahrerassistenzsystemen. Modell zum Kaufverhalten von Endkunden. Wiesbaden: vs Verlag für Sozialwissenschaften.

Backhaus, Klaus; Erichson, Bernd; Plinke, Wulff; Weiber, Rolf (2015): Multivariate Analysemethoden. Eine anwendungsorientierte Einführung. Berlin: Springer.

Bertelsmann (2017): Automatisiertes Fahren. Aktuelle Einstellungen in

Deutschland. Gütersloh: Bertelsmann-Stiftung. Online verfügbar unter https://www.bertelsmann-stiftung.de/fileadmin/files/BSt/Publikationen/ GrauePublikationen/Factsheet_LK_Automatisiertes-Fahren_AktuelleEinstellungen-in-Deutschland_2017.pdf, zuletzt geprüft am 08. 05.2018. Dannheim, Clemens; Mader, Markus; Icking, Christian; Loewenau, Jan; Massow, Kay (2013): A novel approach for the enhancement of cooperative ACC by deriving real time weather information. $16^{\text {th }}$ International IEEE Conference on Intelligent Transportation Systems, ITSC 2013. The Hague, New York: IEEE, S. 2207-2211.

Deloitte (2017): What's ahead for fully autonomous driving. Consumer opinions on advanced vehicle technology. Online verfügbar unter https://www2. deloitte.com/content/dam/Deloitte/de/Documents/manufacturing/ us-manufacturing-consumer-opinions-on-advanced-vehicle-technology.pdf, zuletzt geprüft am 08. 05.2018.

Enquete-Kommission (1998): Abschlussbericht der Enquete-Kommission „Schutz des Menschen und der Umwelt - Ziele und Rahmenbedingungen einer nachhaltig zukunftsverträglichen Entwicklung“. Deutscher Bundestag: Drucksache 13/11200. Online verfügbar unter http://dipbt.bundestag.de/ doc/btd/13/112/1311200.pdf, zuletzt geprüft am 08.05.2018.

Ernst \& Young (2017): Autonomes Fahren in Deutschland. Ergebnisse einer Befragung von 1.000 Verbrauchern. Eschborn: Ernst \& Young GmbH. Online verfügbar unter http://www.ey.com/Publication/vwLUAssets/ey-autonomesfahren-in-deutschland/\$FILE/ey-autonomes-fahren-in-deutschland.pdf, zuletzt geprüft am 08. 05.2018.

Flick, Uwe (2016): Qualitative Sozialforschung. Eine Einführung. Hamburg: Rowohlt. Fraedrich, Eva; Lenz, Barbara (2014): Autonomes Fahren. Mobilität und Auto in der Welt von morgen. Ausblick zur Akzeptanz des autonomen Fahrens im Projekt "Villa Ladenburg“ der Daimler und Benz Stiftung. In: TATuP - Zeitschrift für Technikfolgenabschätzung in Theorie und Praxis 23 (1), S. 46-53. 
Haböck, Ulrich; Klier, Janina; Schwenninger, Jochen; Maier, Stefan (2016): Systemadaption als Schlüssel für das automatisierte Fahren. In: Automobiltechnische Zeitschrift (ATZ) 118 (4), S. 26-31.

Hartwich, Franziska; Beggiato, Matthias; Krems, Josef F. (2018): Driving comfort, enjoyment, and acceptance of automated driving. Effects of drivers' age and driving style familiarity. In: Ergonomics.

Haug, Sonja et al. (2017): Identifikation von Fahrtypen. Ein empirischer Ansatz zur Klassifikation von Fahrtypen anhand von Selbst- und Fremdwahrnehmung bei Testfahrten. Begleitstudie zur Entwicklung eines adaptiven Fahrassistenzsystems. Beitrag zur 2. OTH Forschungsclusterkonferenz, OTH Regensburg, 18. 01.2017, Tagungsdokumentation, S. 176-181. Online verfügbar unter https:// www.elektromobilitaet-regensburg.de/uploads/media/Haug_u.a._2017_ Projekt_Fahrtypen_-_Beitrag_OTH_Clustertagung_2017.pdf, zuletzt geprüft am 08. 05.2018

König, Winfried; Weiß, Karl-Ernst; Mayser, Christoph; (2002): S.A.N.T.O.S. Projekt. Situations-angepasste und Nutzer-Typ-zentrierte Optimierung von Systemen zur Fahrerunterstützung. Online verfügbar unter http://edok01.tib.uni-hannover.de/edoks/e01fb02/373006306.pdf, zuletzt geprüft am 08. 05.2018.

Lohre, Dirk; Bernecker, Tobias; Stock, Wilfried, Düsseldorf, Karl (2014): ZFZukunftsstudie Fernfahrer 2.0. Der Mensch im Transport- und Logistikmarkt. Heilbronn: Institut für Nachhaltigkeit in Verkehr und Logistik der Hochschule Heilbronn. Online verfügbar unter http://www.zf-zukunftsstudie.de/ wp-content/uploads/2014/08/ZF-Zukunftsstudie-2014-Gesamt.pdf, zuletzt geprüft am 08.05.2018.

Pauli, Christine (2014): Ratingverfahren. In: Journal für LehrerInnenbildung 6 (1), S. 56-59.

Payre, William; Cestac, Julia; Delhomme, Patricia (2014): Intention to use a fully automated car. Attitudes and a priori acceptability. In: Vehicle Automation and Driver Behaviour 27 (Part B), S. 252-263.

Reagan, Ian; Cicchino, Jessica; Kerfoot, Laura; Weast, Rebecca (2018): Crash avoidance and driver assistance technologies. Are they used? In: Transportation Research Part F: Traffic Psychology and Behaviour 52, S. 176-190. DOI: 10.1016/j.trf.2017.11.015.
Schnell, Rainer; Hill, Paul; Esser, Elke (2013): Methoden der empirischen Sozialforschung. München: Oldenbourg.

Verberne, Frank; Ham, Jaap; Midden, Cees (2012): Trust in smart systems.

Sharing driving goals and giving information to increase trustworthiness and acceptability of smart systems in cars. In: Human Factors 54 (5), S. 799-810.

Wang, Jianqiang; Li, Keqiang; Zhang, Dezhao; Zhang, Lei; (2014): An adaptive longitudinal driving assistance system based on driver characteristics. In: IEEE Transactions on Intelligent Transportation Systems 14 (1), S. 1-12. Woisetschläger, David (2015): Machtauswirkungen des automatisierten Fahrens. In: Markus Maurer, Christian Gerdes, Barbara Lenz und Hermann Winner (Hg.): Autonomes Fahren. Technische, rechtliche und gesellschaftliche Aspekte. Berlin: Springer Vieweg, S. 709-732.

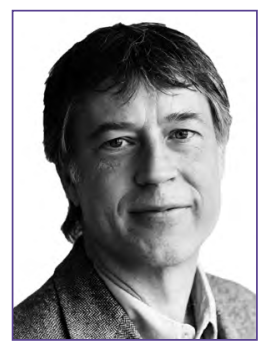

\section{PROF.DR. KARSTEN WEBER}

ist Ko-Leiter des Instituts für Sozialforschung und Technikfolgenabschätzung (IST) der OTH Regensburg sowie Honorarprofessor für Kultur und Technik an der BTU Cottbus-Senftenberg. Er beschäftigt sich derzeit mit individuellen und gesellschaftlichen Auswirkungen von luK-Technologie sowie mit wertebasierter Gestaltung von Technik insbesondere im Gesundheitsbereich.

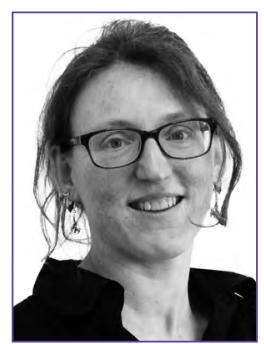

PROF.DR. SONJA HAUG

ist Ko-Leiterin des Instituts für Sozialforschung und Technikfolgenabschätzung (IST) sowie Professorin für Methoden der Sozialforschung und Sozioinformatik der OTH Regensburg. Sie forscht unter anderem zur Technikakzeptanz und -nutzung in den Bereichen Gesundheit, Energie sowie luK-Technologien.

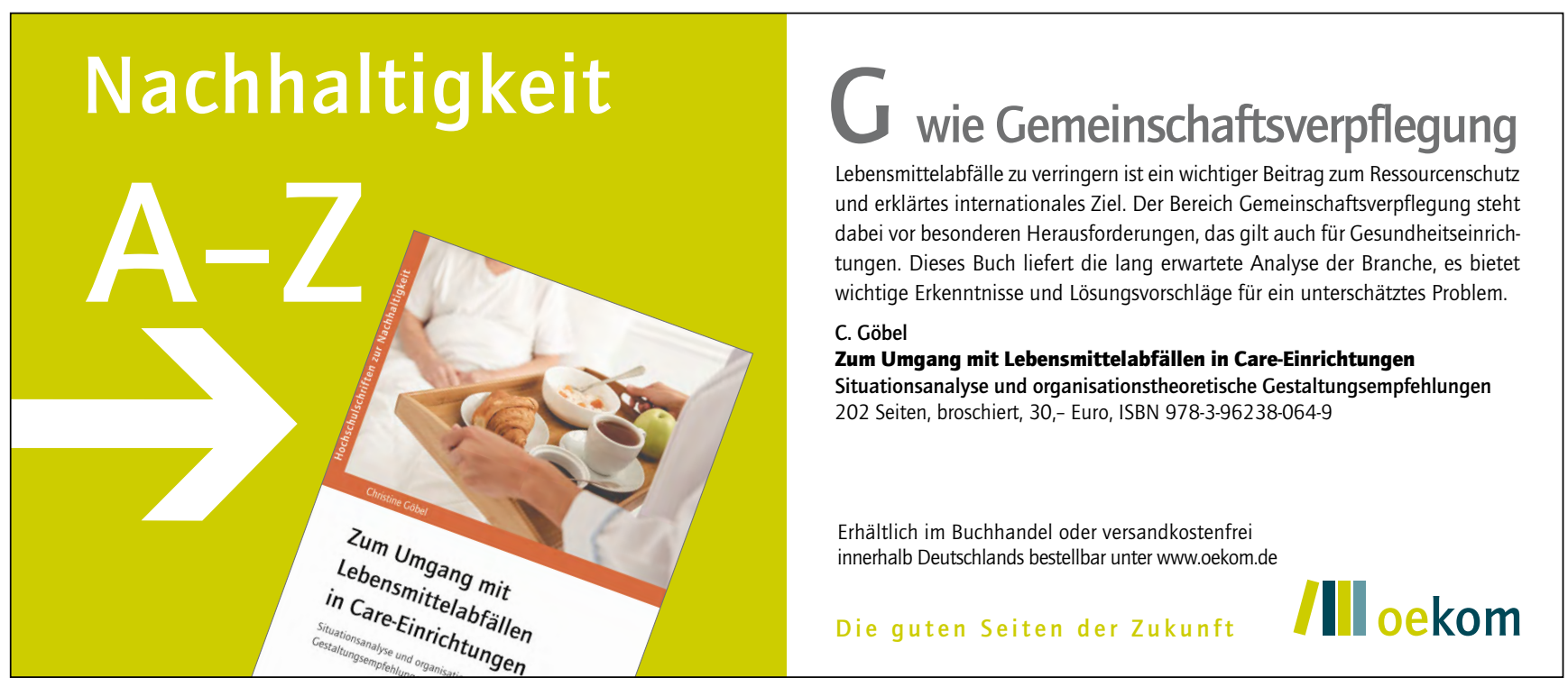




\title{
Gesamtwirtschaftliche Kosten- einsparpotenziale durch das automatisierte Fahren
}

\author{
Eine ökonomische Abschätzung für Deutschland
}

\author{
Michael Roos, Ruhr-Universität Bochum, Fakultät für Wirtschaftswissenschaft, Lehrstuhl für Makroökonomik, Universitätsstraße 150, 44801 Bochum \\ (michael.roos@rub.de), (D) orcid.org/0000-0002-5465-9893 \\ Marvin Siegmann, Ruhr-Universität Bochum, Fakultät für Wirtschaftswissenschaft, Lehrstuhl für Makroökonomik \\ (marvin.siegmann@rub.de), (D) orcid.org/0000-0003-3954-768X
}

In dieser Studie wird abgeschätzt, welche gesamtwirtschaftlichen Kosteneinsparungen das automatisierte Fahren für Deutschland bringen könnte. Durch automatisiertes Fahren können Unfälle und Staus vermieden werden. Zudem ist zu erwarten, dass der Parkverkehr in den Innenstädten zurückgeht. Insgesamt ergibt unsere Abschätzung ein Einsparpotenzial von 24,9 Mrd. Euro pro Jahr bei einer mittleren Marktdurchdringung. Dies entspricht etwa 0,8\% des deutschen Bruttoinlandsprodukts im Jahr 2016. Gemessen am Wirtschaftswachstum von 1,9\% im Jahr 2016 ist dies erheblich. Von besonderem Interesse ist auch die Kostenersparnis je Pkw, da somit private Kosten der Anschaffung mit sozialen Erträgen verglichen werden können. Im mittleren Szenario erhalten wir eine Kostenersparnis von 1.351 Euro pro Jahr und Pkw.

\section{Macroeconomic Cost Saving Potentials through Automated Driving An Economic Estimation for Germany}

This study estimates the macroeconomic cost saving potentials, that might be achieved through automated driving in Germany. The new technologies of autonomous vehicles can contribute to accident and congestion reductions and might also lower parking problems in inner cities. Our assessment shows an annual cost saving potential of 24.9 bill. Euro at mid-level market penetration. This corresponds to $0.8 \%$ of the German GDP in 2016, which is considerably high compared to the economic growth of $1.9 \%$ in the same year. Of particular interest here are also cost savings per car in order to compare private costs with socially realized benefits. We estimate cost savings of 1,351 Euro per year and car at mid-level market penetration.

Keywords: autonomous vehicles, automated driving, safety, congestion, macroeconomic cost reduction

This is an article distributed under the terms of the Creative Commons Attribution License CCBY 4.0 (https://creativecommons.org/licenses/by/4.0/)

https://doi.org/10.14512/tatup.27.2.23

Submitted: 09. 03.2018. Peer reviewed. Accepted: 28. 05.2018
Seit einiger Zeit erproben zahlreiche Automobilhersteller und Technologieunternehmen die Funktionsweise autonomer Fahrzeuge, die durch den Einsatz technischer Assistenzsysteme ohne menschliches Einwirken operieren können. Autonome Fahrzeuge versprechen eine Aufwertung der Fahrtzeit, ein deutlich erhöhtes Sicherheitsniveau, eine zeit- und kraftstoffeffizientere Fortbewegung bei reduziertem Emissionsausstoß und sollen auch älteren und körperlich beeinträchtigten Personen den $\mathrm{Zu}$ gang zum Individualverkehr ermöglichen. Die serienreife Einführung dieser neuen Fahrzeugart könnte sowohl die Personenbeförderung als auch den Gütertransport auf der Straße revolutionieren. Während in der Bevölkerung eine gewisse Skepsis gegenüber selbstfahrenden Autos besteht, erwarten Vertreter der Automobilbranche, von Interessensverbänden und aus der Politik erhebliche gesellschaftliche Vorteile von der neuen Technologie. So schrieb der Bundesverkehrsminister Alexander Dobrindt am 24. 6. 2015 in der Frankfurter Allgemeinen Zeitung: „Deshalb ist es wichtig, dass Deutschland bei der nächsten großen Mobilitätsrevolution vorangeht: dem autonomen und vernetzen Fahren. Damit bekommt Mobilität eine völlig neue Dimension. Das Auto wird zum Third Place, einem weiteren Lebensmittelpunkt neben Büro und Zuhause. Das Fahren entwickelt sich von der zweckgebundenen Notwendigkeit zu einem neuen, produktiven Zeitfenster. Gleichzeitig macht Echtzeit-Daten-Kommunikation zwischen Autos und Infrastruktur den Verkehr vorhersehbar und vermeidet Stau und Unfälle.“

In dieser Studie wird abgeschätzt, welche gesamtwirtschaftlichen Kosteneinsparungen das automatisierte Fahren für Deutschland bringen könnte. Wir beschränken uns dabei auf Kosten, die mit von Menschen gesteuerten Fahrzeugen durch Unfälle, Staus und das Parken in Ballungsräumen entstehen und durch den Einsatz von selbstfahrenden Autos vermieden werden könnten. Weitere Auswirkungen, wie etwa die Umgestaltung innerstädtischer Nutzungsflächen als Folge eines verringerten Ver- 
kehrsaufkommens oder Veränderungen der produzierten Emissionsmengen an Treibhausgasen, werden an dieser Stelle ausgeklammert. Ebenfalls ausgespart werden Beschäftigungs- und Einkommenseffekte in der Automobilindustrie und anderen von der Technologie betroffenen Branchen wie der Logistik oder dem Personentransportwesen. Die vernachlässigten Auswirkungen sind wahrscheinlich von erheblicher Größenordnung, jedoch ist ihre Ermittlung schwierig und kann nicht im Rahmen dieser Studie geleistet werden. Die hier ermittelten Kosteneinsparpotentiale sollten daher nicht als Ergebnis einer umfassenden Kosten-Nutzen-Analyse interpretiert werden, sondern als erste Abschätzung möglicher Größenordnungen der direkten Effekte. unter anderem auf gegenwärtigen Daten für das jährliche Unfallund Stauaufkommen sowie Kraftstoffverbräuchen verschiedener Fahrzeugarten und berücksichtigt Schätzungen darüber, welchen Einfluss menschliches Verhalten auf diese Größen hat. Unter der Annahme, dass die Parameter und das Verhalten gleichbleiben, werden die Daten dann herunterskaliert, indem ein Teil der von Menschen gesteuerten Fahrzeuge durch autonome Fahrzeuge ersetzt wird. Diese Methode vernachlässigt weitgehend, dass sich Preise und Kosten und damit Verhalten auf Grund der neuen Technologie ändern können. Solche Änderungen könnten nur in einem Modell abgebildet werden. Daher ist unsere Studie auch nicht als Zukunftsprojektion zu verstehen, sondern

Insgesamt ergibt unsere Abschätzung ein Einsparpotenzial von 24,9 Mrd. Euro pro Jahr bei einer mittleren Marktdurchdringung autonomer Fahrzeuge. Dies entspricht etwa 0,8\% des deutschen Bruttoinlandsprodukts im Jahr 2016.

Die makroökonomischen Aspekte des autonomen Autofahrens sind bisher kaum erforscht. So ist bemerkenswert, dass der von der Daimler und Benz Stiftung geförderte Sammelband zum autonomen Fahren (Maurer et al. 2015) sich auf die technischen, rechtlichen und gesellschaftlichen Aspekte konzentriert und die ökonomischen Aspekte weitgehend außen vorlässt. Unseres Wissens gibt es bisher keine Abschätzung gesamtwirtschaftlicher Auswirkungen des autonomen Fahrens für Deutschland. Auch die internationale Forschungsliteratur bietet bisher wenig Erkenntnisse zu ökonomischen Fragen. Der Unternehmensberatungszweig strategy \& von PricewaterhouseCoopers nimmt sich in seinem Bericht zum autonomen Fahren (strategy \& 2016) eine betriebswirtschaftliche Analyse der Chancen und Risiken der globalen Automobilindustrie vor. Eine Delphi-Studie im Rahmen des EU-geförderten Projekts CityMobil2 (Sessa et al. 2015) betrachtet zwar auch ökonomische Effekte, jedoch nur qualitativ und ohne den Versuch, ökonomische Größen quantitativ abzuschätzen.

Die einzige uns bekannte Studie, die versucht, den makroökonomischen Nutzen des autonomen Autofahrens zu ermitteln, ist Fagnant und Kockelman (2015). Diese Studie extrapoliert für die USA, welche gesamtwirtschaftliche Kosten durch Unfälle, Staus und Parken bei unterschiedlicher Markdurchdringung autonomer Autos eingespart werden könnten. Wir wenden diese Methode auf deutsche Daten an und nehmen damit die erste makroökonomische Abschätzung für Deutschland vor. Dabei berechnen wir verschiedene Szenarien, indem wir jeweils einen niedrigen, einen mittleren und einen hohen Marktanteil autonomer Fahrzeuge unterstellen. Die Berechnungen basieren dabei auf Extrapolationen aktueller Daten, was für eine erste Abschätzung akzeptabel erscheint. Diese Methode basiert als Vergleich der gegenwärtigen Situation mit und ohne selbstfahrende Autos.

Insgesamt ergibt unsere Abschätzung ein Einsparpotential von 24,9 Mrd. Euro pro Jahr bei einer mittleren Marktdurchdringung. Dies entspricht etwa $0,8 \%$ des deutschen Bruttoinlandsprodukts im Jahr 2016. Gemessen am Wirtschaftswachstum von 1,9\% im Jahr 2016 ist dies erheblich. Fagnant und Kockelman (2015) kommen für die USA auf eine ähnliche Größenordnung. Von besonderem Interesse ist auch die Kostenersparnis je Pkw, da somit private Kosten der Anschaffung mit sozialen Erträgen verglichen werden können. Im mittleren Szenario erhalten wir eine Kostenersparnis von 1.351 Euro pro Jahr und Pkw.

\section{Potenziale der Unfallvermeidung}

\section{Allgemeine Unfallstatistiken}

Zur Bestimmung möglicher Potenziale der Unfallvermeidung durch autonome Fahrzeuge gehen wir von den gegenwärtigen deutschen Unfallstatistiken aus. Die Polizei erfasste im Jahr 2016 etwa 2,56 Millionen Unfälle im deutschen Straßenverkehrsnetz (Institut der deutschen Wirtschaft Köln, 2016), bei denen 3.206 Personen ums Leben kamen (Statistisches Bundesamt, 2017). Etwa $86 \%$ aller Unfallursachen können auf menschliches Fehlverhalten von Kraftfahrzeug- und Fahrradfahrern und $4 \%$ auf ein solches von Fußgängerinnen und Fußgängern zurückgeführt werden. Allgemein schlechte Straßenzustände sowie widrige Wetterverhältnisse sind nur in $9 \%$ und technische Mängel in 1\% der Fälle die Ursache (Hütter 2013, S. 38). Baum et al. (2010) beziffern in einem Bericht die volkswirtschaftlichen Kosten der Straßenverkehrsunfälle in Deutsch- 
land. Die Berechnungen umfassen nicht nur die direkten Reproduktionskosten der Sachschäden, sondern auch Polizei- und Rechtssprechungskosten, Verwaltungskosten der Versicherungen, Ressourcenausfälle durch Personenschaden, Rückgänge außermarktwirtschaftlicher Wertschöpfung, humanitäre Verluste und weitere Kostenstellen. Demnach lag der volkswirtschaftliche Schaden im Jahr 2005 bei 31,477 Mrd. Euro (Baum et al. 2010, S. 87) ${ }^{1}$. Setzt man diesen Wert aus dem Jahr 2005 mit den korrespondieren-

\begin{tabular}{|l|r|r|r|}
\hline \multicolumn{1}{|c|}{ Marktanteil autonomer Fahrzeuge } \\
\hline Anzahl autonomer Fahrzeuge & \multicolumn{1}{|c|}{$10 \%$} & \multicolumn{1}{l|}{$\mathbf{5 0} \%$} & $\mathbf{9 0 \%}$ \\
\hline Gerettete Leben (p. a.) & 4.684 .363 & 19.070 .478 & 26.535 .675 \\
\hline Weniger Unfälle (p. a.) & 159 & 1.082 & 2.467 \\
\hline $\begin{array}{l}\text { Volkswirtschaftliche Kostenersparnisse } \\
\text { (in Euro, p. a.) }\end{array}$ & 1.787 .152 .630 & 12.185 .131 .566 & 27.782 .099 .970 \\
\hline Je autonomen Fahrzeug (in Euro, p. a.) & 382 & 639 & 1.989 .409 \\
\hline
\end{tabular}

Tab.1: Unfallvermeidungspotenziale durch autonome Fahrzeuge.
Quelle: Eigene Darstellung den Unfallzahlen ins Verhältnis und überträgt dieses auf das Jahr 2016, ergeben sich geschätzte Kosten in Höhe von 36,104 Mrd. Euro. Damit machen die Unfallkosten knapp 1,15\% des Bruttoinlandsproduktes von Deutschland aus.

\section{Unfallvermeidung}

Autonome Fahrzeuge können menschliche Fehler als Unfallursache eliminieren, da sie Verkehrssituationen besser überblicken, aggressive Fahrweisen ausgeschlossen werden und Gefahrensituationen durch den Datenaustausch mit anderen Fahrzeugen und der Infrastruktur antizipiert werden können. Es wird angenommen, dass ein einziges autonomes Fahrzeug, es sei also ein Marktanteil von quasi $0 \%$ unterstellt, die Unfallwahrscheinlichkeit mit einem anderen Fahrzeug um den Faktor 0,5 verringern kann. Denn es kann zwar eigene Fahrfehler im besten Fall vermeiden, nicht aber die Fehler des anderen (menschlichen) Fahrers korrigieren. Eine Unfallwahrscheinlichkeit von 0,5 ist auch insofern annehmbar, als dass sich die Unfallschuld im Mittel gleichmäßig auf alle Kraftfahrer verteilt und somit durch ein autonomes Fahrzeug bei einem drohenden Unfall mit zwei Fahrzeugen halbiert. Ausgehend von dieser Annahme werden nun die Unfallwahrscheinlichkeiten für verschiedene Marktanteile autonomer Fahrzeuge ermittelt. Wir folgen anderen Studien, die bei vergleichbaren Abschätzungen Marktanteile in Höhe von 10, 50 und $90 \%$ gewählt haben (Fagnant und Kockelman 2015; Shladover et al. 2012).

Bei einem Marktanteil von 10\% trifft ein autonomes Fahrzeug in praktisch $10 \%$ der Fälle ${ }^{2}$ auf ein weiteres seiner Art, und ein Unfall, der auf menschliche Fehler zurückführbar wäre, könnte vermieden werden. Die oben unterstellte Unfallwahrscheinlichkeit von 0,5 bei einem Marktanteil von nahe $0 \%$ verringert sich also um $10 \%$ auf 0,45 . Vermieden wird ein Unfall daher mit einer Wahrscheinlichkeit von $1-0,45=0,55$. Der Berechnungsansatz könnte alternativ auch wie folgt lauten: In $10 \%$ der Fälle trifft ein autonomes Fahrzeug auf ein weiteres seiner Art, so dass ein durch menschliches Versagen herbeige-

1 Leider gibt es keine aktuelleren Zahlen, was am hohen Berechnungsaufwand liegen dürfte.

2 Das eigene Fahrzeug ist in den $10 \%$ inbegriffen. führter Unfall mit der Wahrscheinlichkeit 1 vermieden wird. In allen anderen Fällen, also $90 \%$, verringert sich die Wahrscheinlichkeit eines Unfalls, wie beschrieben, um 50\%, also $0,1 \times 1$ $+0,9 \times 0,5=0,55$. Für einen Marktanteil von $50 \%$ gilt ana$\log 0,5+0,5 \times 0,5=0,75$ und für einen von $90 \%$ gilt $0,9+0,1$ $\times 0,5=0,95$.

Die in menschlichen Fehlern begründeten Unfälle könnten also bei einem Marktanteil von 10\% autonomer Fahrzeuge um den Faktor 0,55 verringert werden. Der Ansatz lautet daher:

$$
\begin{aligned}
& 2.585 .327(\text { Unfälle }) \times 90 \%(\text { menschliche Fehler }) \times 10 \% \\
& (\text { Marktanteil AF }) \times 0,55(\text { Unfallvermeidungsfaktor }) \\
& =127.974
\end{aligned}
$$

In der Konsequenz könnten jährlich fast 128.000 Unfälle verhindert werden. Die volkswirtschaftlichen Kostenersparnisse ergeben sich analog und liegen bei einer Marktdurchsetzung von $10 \%$ bei

$$
\begin{aligned}
& 36,104 \text { Mrd. } €(\text { Volksw.Schaden }) \times 90 \%(\text { mensch }- \\
& \text { liche Fehler }) \times 10 \%(\text { Marktanteil AF }) \times 0,55(\text { Unfall- } \\
& \text { vermeidungsfaktor }) \approx 1,787 \text { Mrd. } €
\end{aligned}
$$

Die korrespondierenden Werte für die Marktanteile von 50\% und $90 \%$ werden, auf ganze Zahlen gerundet, in Tabelle 1 aufgeführt, ebenso wie die geretteten Leben, die analog ermittelt wurden.

Darüber hinaus sind die volkswirtschaftlichen Kostenersparnisse pro autonomem Fahrzeug von besonderem Interesse. In der Bundesrepublik Deutschland gab es zum 1. Januar 2017 einen Fahrzeugbestand von 45.803.560 Personenkraftwagen, 78.949 Kraftomnibussen, 2.911.907 Lastkraftwagen und 2.170.335 Sattelzugmaschinen, was einer Gesamtanzahl von 48.996.400 Fahrzeugen entspricht (Kraftfahrt-Bundesamt, 2017, S. 1). Die Anzahl aller autonomen Fahrzeuge wird durch Multiplikation dieses Bestandes an herkömmlichen Kraftfahrzeugen mit dem jeweiligen Marktanteil autonomer Fahrzeuge bestimmt. Es ist wahrscheinlich, dass sich auf Grund von Kosten- und Verhaltensänderungen der Fahrzeugbestand ändern wird, jedoch ist eine Abschätzung dieser Änderung schwierig. Sinkende Kosten, z. B. durch Kraftstoffeinsparungen und geringere Versicherungsprämien, könnten zu mehr Fahrzeugen führen. Car-Sha- 
ring, das vor allem in den Innenstädten attraktiv ist, könnte vor allem die Zahl der Personenkraftwagen verringern. Wir ignorieren hier mögliche Änderungen bei den anderen Fahrzeugklassen und nehmen eine Verringerung der Personenkraftwagen durch Car-Sharing an.

Durch Car-Sharing können zum einen Kosten gespart werden, weil Versicherungs- und Wartungskosten auf mehrere Fahrer aufgeteilt werden können (Barter 2013). Zum anderen wären weniger Fahrzeuge erforderlich, um dieselbe Transportleistung zu erzielen. Eine Studie der Universität von Columbia hat beispielsweise gezeigt, dass eine Flotte von etwa 9.000 autonomen Automobilen ausreiche, um jedes Taxi in New York City tenwert von 7,695 Euro, im Rahmen des Arbeitsweges von 9,18 Euro und bei Dienstfahrten von 25,07 Euro (HEATCO $2005)^{4}$. Im Folgenden wird zwischen privaten Fahrten und solchen für berufliche Zwecke differenziert, letztere beiden Werte werden deshalb zu 17,125 Euro gemittelt. Nach einem Bericht des Umweltbundesamtes liegt der Verkehrsaufwand für berufliche Zwecke bei etwa 18,1\% (Umweltbundesamt 2012, S. 28). Die Potenziale der Stauvermeidung ergeben sich vor allem aus dem monetären Gegenwert der im Stau verbrachten Zeit und den Kosten des im Stau verschwendeten Kraftstoffs. Anhand der genannten Statistiken ermitteln wir nachfolgend diese Kosten.

\section{Autonome Fahrzeuge können menschliche Fehler als Unfallursache} eliminieren, da sie Verkehrssituationen besser überblicken, nicht aggressiv fahren und durch Datenaustausch Gefahrensituationen antizipieren.

zu ersetzen. Die Verbraucher würden im Schnitt nur $36 \mathrm{Se}-$ kunden auf ein freies Fahrzeug warten und etwa 50 US-Cent pro gefahrene Meile zahlen. Eine solche Transportation Cloud könnte aufgrund der hohen Verfügbarkeit und geringeren Kosten den Besitz eines eigenen Autos für manche überflüssig machen und eine attraktive Alternative zum klassischen Personennahverkehr darstellen (Kanter, 2015). Fagnant und Kockelman (2015, S. 175) nehmen an, dass sich vor allem durch Car-Sharing von autonomen Fahrzeugen die Gesamtzahl der Pkw bei einem Marktanteil von $10 \%$ um $4,7 \%$, bei $50 \%$ um 23,7 $\%$ und bei $90 \%$ um 42,6\% verringert. Wir übernehmen diese Annahme und berechnen die Anzahl der autonomen Fahrzeuge anhand des jeweiligen Marktanteils bezogen auf die verminderte Gesamtzahl der Pkw.

\section{Potenziale der Stauvermeidung}

\section{Allgemeine Staustatistiken}

In Deutschland gibt es, gemessen an der Zahl der gültigen Führerscheine, etwa 36 Millionen Kraftfahrer (Statista 2017e), von denen jeder im bundesweiten Durchschnitt jährlich etwa 30 Stunden im Stau steht (INRIX 2016). Daraus ergibt sich eine zeitliche Staubelastung von insgesamt 1,08 Mrd. Stunden. Dabei werden alleine in Staus auf Autobahnen rund 321,792 Millionen Liter Kraftstoffe vergeudet (Die Welt 2009)3. Der durchschnittliche Preis für einen Liter Benzin lag 2016 bei 129,6 Cent (Statista 2017 c) und der für einen Liter Diesel bei 107,2 Cent (Statista 2017d). Jede Zeitstunde, die im Stau verbracht wird, hat im Mittel bei Privatfahrten einen wirtschaftlichen Kos-

3 Die Daten wurden hier extrapoliert und an das Verkehrsaufkommen in 2016 angepasst.

\section{Stauvermeidung}

Die technischen Assistenzsysteme autonomer Fahrzeuge stellen sicher, dass die Fahrgeschwindigkeit optimal an die Verkehrslage angepasst und ineffizientes Brems- und Beschleunigungsverhalten vermieden wird. Hoch frequentierte Streckenabschnitte können durch den Datenaustausch mit vorausfahrenden Fahrzeugen frühzeitig erkannt und umfahren werden. Diese Funktionen führen zu Einsparungen der Kraftstoffmengen. Hier gelten also viele der Vorteile, die bereits zuvor bei den Potenzialen der Unfallvermeidung angeführt wurden. Stern et al. (2017) konnten in einem Praxisversuch zeigen, dass bereits ein einziges autonomes Fahrzeug in einem Testfeld mit insgesamt zwanzig Fahrzeugen den Verkehrsfluss signifikant verbessern kann. Die Potenziale der Stauvermeidung fallen also nicht nur exklusiv autonomen Fahrzeugen zu, sondern allen Verkehrsteilnehmern. Die Kraftstoffeffizienz verbessert sich schätzungsweise um 23-39\% und die Fahrgeschwindigkeit bei stockendem Verkehr erhöht sich um 8-13\%, je nach Marktanteil autonomer Fahrzeuge (Fagnant und Kockelman 2015, S. 170). Die StraBenauslastung könnte unter anderem durch das autonome Fahren in Konvois bei einem Marktanteil von $10 \%$ um $1 \%$, bei $50 \%$ um $21 \%$ und bei $90 \%$ um $80 \%$ erhöht werden (Shladover et al. 2012). Fagnant und Kockelman (2015) formulieren eine vorsichtigere Schätzung und tragen dabei auch gegenteiligen Effekten Rechnung, wie beispielsweise der Erhöhung des allgemeinen Verkehrsaufkommens aufgrund der steigenden Mobilität. Sie unterstellen bei einem Marktanteil von 10\% Staureduktionsfaktoren in Höhe von $15 \%$ und $5 \%$, bei $50 \%$ Markt-

4 Die Verwendung von Zeitkostensätzen unterstellt eine andernfalls effiziente Nutzung dieser Zeit, die nicht gegeben sein muss. Daher werden die Zeitkosten bei diesem Ansatz tendenziell überschätzt. Wir danken einem der Gutachter für den Hinweis. 
durchsetzung von $35 \%$ und $10 \%$ und bei $90 \%$ Markterfolg von $60 \%$ und $15 \%$, jeweils differenziert für Autobahnen und andere Straßen außerhalb von Ortschaften sowie dem innerstädtischen Bereich.

\section{Zeitersparnis}

Eine Verbesserung des Verkehrsflusses führt zu impliziten Zeitersparnissen. Bei einem Marktanteil autonomer Fahrzeuge in Höhe von $10 \%$ könnte sich das Stauaufkommen also für alle Verkehrsteilnehmer außerorts ${ }^{5}$ um $15 \%$ und im innerstädtischen Bereich um $5 \%$ reduzieren (Fagnant und Kockelman 2015). Laut Umweltbundesamt (2011) erfolgt etwa 30\%

\begin{tabular}{|c|c|c|c|}
\hline & \multicolumn{3}{|c|}{ Marktanteil autonome Fahrzeuge } \\
\hline & $10 \%$ & $50 \%$ & $90 \%$ \\
\hline Anzahl autonomer Fahrzeuge & 4.684 .363 & 19.070 .478 & 26.535 .675 \\
\hline Zeitersparnis (in Stunden, p. a.)* & 129.943 .189 & 297.857 .972 & 503.744 .349 \\
\hline Kostenersparnis Zeit (in Euro, p.a.) & 1.221.826.306 & 2.800 .690 .895 & 4.736.593.766 \\
\hline Kraftstoffeinsparungen (in Litern, p.a.)* & 38.717 .295 & 88.748 .437 & 150.093 .427 \\
\hline Kostenersparnis Kraftstoffe (in Euro, p.a.) & 47.056 .826 & 107.864 .452 & 182.422 .877 \\
\hline Kostenersparnis gesamt (in Euro, p. a.) & 1.268 .883 .133 & 2.908.555.347 & 4.919.016.643 \\
\hline Je autonomen Fahrzeug (in Euro, p. a.) & 271 & 153 & 185 \\
\hline
\end{tabular}

des Straßengüterverkehrs innerorts. Werden diese Werte mit den bereits angeführten Fahrzeugbeständen gewichtet, kann für den gesamten Verkehr ein Wert von schätzungsweise $29,7 \%$ zugrunde gelegt werden. Dementsprechend findet 70,3\% des deutschen Verkehrs außerorts statt. Die effektive Zeitersparnis durch autonome Systeme läge bei einem Marktanteil von 10\% bei

$$
\begin{aligned}
& (1,08 \mathrm{Mrd} . \mathrm{h}(\text { Stau }) \times 15 \%(\text { Faktor außerorts }) \times 70,3 \%) \\
& +(1,08 \mathrm{Mrd} . \mathrm{h}(\text { Stau }) \times 5 \%(\text { Faktor innerorts }) \times 29,7 \%) \\
& \approx 130 \text { Mio. } \mathrm{h}(\text { Zeitersparnis })
\end{aligned}
$$

In einem nächsten Schritt wird dieser Wert mit den zuvor erwähnten Kosten einer Staustunde verrechnet. Für einen Anteil von $18,1 \%$ gilt der Kostensatz einer Fahrt für berufliche Zwecke, für den Rest gilt der einer privaten Fahrt. Die Rechnung lautet:

(130 Mio. $\mathrm{h} \times 18,1 \%$ (Berufl. Zwecke $) \times 17,125 €$

(Kosten berufl. Zwecke $))+(130$ Mio.h $\times(1-18,1 \%)$

$\times 7,695 €($ Kosten Privatf. $)) \approx 1,222$ Mrd. $€$

Bei einem Marktanteil von 10\% könnte die zeitliche Staubelastung um rund 130 Mio. Stunden reduziert werden, was einem finanziellen Gegenwert von 1,222 Mrd. Euro entspricht.

\section{Kraftstoffeinsparungen}

Bei der Bemessung der Kraftstoffeinsparungen durch Staureduzierungen wird zunächst analog zu [3] verfahren:

$$
\begin{aligned}
& (321,792 \text { Mio. } 1 \text { (Treibstoff }) \times 15 \% \text { (Faktor } \\
& \text { außerorts }) \times 70,3 \%)+(321,792 \text { Mio. } 1 \text { (Treibstoff }) \\
& \times 5 \%(\text { Faktor innerorts }) \times 29,7 \%) \\
& \approx 38,717 \text { Mio. } 1 \text { (Treibstoffeinsparungen) }
\end{aligned}
$$

Der Anteil der Pkw mit Dieselmotoren liegt bei 32,2 (Kraftfahrt-Bundesamt 2017, S. 1), unter Lkw bei 88 (Statista 2017 b) und unter Kraftomnibussen bei 97,5\% (Statista 2017 a). Unter Sattelzugmaschinen liegt dieser schätzungsweise bei dem der

5 Wir erfassen alle Straßen außerhalb von Ortschaften, wie Autobahnen und Fernstraßen, aber auch Kreis- und Landstraßen.
Kraftomnibusse. Daraus ergibt sich, gewichtet mit den zuvor angeführten Fahrzeugbeständen der jeweiligen Typklassen, ein Durchschnitt von knapp 36\%. Die in [5] berechneten Spriteinsparungen werden nun anteilig mit den durchschnittlichen Literpreisen für Diesel und Benzin verrechnet:

$$
\begin{aligned}
& (38,717 \text { Mio. } 1 \text { (Spriteinsp. }) \times 36 \%(\text { Anteil Dieself. }) \\
& \times 1,072 €(\text { Literp. Diesel }))+(38,717 \text { Mio. } 1 \text { (Spriteinsp. }) \\
& \times(1-36 \%(\text { Anteil Benziner }) \times 1,296 €(\text { Literp. Benzin })) \\
& \approx 47,057 \text { Mio. } €
\end{aligned}
$$

Insgesamt könnten durch die Stauvermeidung bei einem Marktanteil von $10 \%$, folgernd aus der Addition von 1,222 Mrd. Euro aus den Zeitersparnissen und 47,057 Mio. Euro aus den Kraftstoffeinsparungen, rund 1,269 Mrd. Euro eingespart werden. Tabelle 2 zeigt die Ergebnisse für alle Marktanteilsszenarien.

\section{Potenziale der Parkkostenreduzierung}

Das Parken eines Fahrzeugs ist insbesondere in Ballungsräumen mit Kosten und Zeitaufwand verbunden. In Deutschland liegt der durchschnittliche Preis für die Nutzung eines Parkhauses bei 1,45 Euro pro Stunde (Reichwein 2016). Die Suche nach einem geeigneten Abstellplatz dauert im Durchschnitt zehn Minuten und macht rund ein Drittel des innerstädtischen Verkehrs aus. Zudem werden etwa $40 \%$ aller Unfälle mit Sachschaden beim Ein- und Ausparken verursacht. Einige Automobilhersteller und -zulieferer arbeiten deshalb an technischen Lösungen für das autonome Parken von Fahrzeugen. Die ersten Konzepte werden bereits in praxisnahen Umgebungen erprobt und versprechen eine deutlich erhöhte Effizienz gegenüber dem manuellen Einparken (Zeit Online 2012). Durch die Nutzung der autonomen Fahrfähigkeiten der Fahrzeuge könnten diese darüber hinaus nach Absetzen der Insassen eigenständig günstigere Parkflächen außerhalb des Stadtkerns suchen. Litman (2016) geht davon aus, dass durch eine solche Praxis unter Berücksichtigung der Bau-, Landnutzungs- und Betriebskosten einer Parkfläche 
die jährlichen Aufwendungen für das Parken um etwa $57 \%$ gesenkt werden könnten. Gleichzeitig stehen die innerstädtischen Parkplatzflächen zukünftig für anderweitige Verwendungen, beispielsweise für Wohnraum oder Grünflächen, zur Verfügung.

Die Abschätzungen zu möglichen Potenzialen der Parkkostenreduzierung beziehen sich an dieser Stelle nur auf private Pkw und klammern alle andere Fahrzeugarten aus. Für die nachfolgenden Berechnungen wird deshalb die Anzahl autonomer Pkw und nicht die aller autonomer Fahrzeuge als Basis herangezogen. Wie oben erläutert unterstellen wir eine Verringerung der Anzahl der Pkw durch

\begin{tabular}{|c|c|c|c|}
\hline & \multicolumn{3}{|c|}{ Marktanteil autonome Fahrzeuge } \\
\hline & $10 \%$ & $50 \%$ & $90 \%$ \\
\hline Anzahl autonome Pkw & 4.365 .079 & 17.474 .058 & 23.662.119 \\
\hline $\begin{array}{l}\text { Kostenersparnis Parken pro autonomem } \\
\text { Pkw (in Euro, p. a.) }\end{array}$ & 174 & 174 & 174 \\
\hline Kostenersparnis Parken (in Euro, p.a.) & 759.523 .793 & 3.040 .486 .116 & 4.117.208.723 \\
\hline $\begin{array}{l}\text { Zeitersparnis Parkplatzsuche } \\
\text { (in Stunden, p.a.) }\end{array}$ & 178.968 .250 & 716.436 .384 & 970.146 .883 \\
\hline $\begin{array}{l}\text { Kostenersparnis Parkplatzsuche } \\
\text { (in Euro, p. a.) }\end{array}$ & 1.682 .797 .829 & 6.736 .488 .685 & 9.122 .070 .917 \\
\hline Kostenersparnis gesamt (in Euro, p.a.) & 2.442 .321 .621 & 9.776 .974 .802 & 13.239.279.640 \\
\hline Je autonomen Pkw (in Euro, p.a.) & 560 & 560 & 560 \\
\hline
\end{tabular}

Tab.3: Zeit- und Kostenersparnisse durch die autonome Parkplatzsuche.

Quelle: Eigene Darstellung

Car-Sharing.

Fagnant und Kockelman (2015) nehmen für die amerikanischen Autofahrer pauschal eine Ersparnis in Höhe von 250 USD pro autonomen Pkw an, weil autonome Fahrzeuge selbständig auf günstigeren Stellplätzen parken ${ }^{6}$. Laut INRIX (2017) liegen die durchschnittlichen Parkkosten für zwei Stunden in den USA bei 4 USD und in Deutschland bei $3 €$. Skaliert man die pauschale Kosteneinsparung von 250 USD mit dem Verhältnis der Parkkosten in Deutschland und in den USA erhält man eine Kosteneinsparung in Deutschland von 187,5€ pro Jahr. Dieser Wert ist vielleicht etwas hoch. Laut einer Studie von APCOA (2013) zahlen deutsche Autofahrer pro Jahr durchschnittlich $60 €$ für Parkhäuser. Zudem fallen im Schnitt $16 €$ pro Jahr an Kosten für Strafzettel wegen Falschparkens an. INRIX (2017) gibt an, dass Autofahrer pro Jahr $98 € \mathrm{zu}$ viel an Parkgebühren bezahlen, um Strafzettel zu vermeiden. Zusammen ergibt dies Kosten in Höhe von $174 €$, die überwiegend in Innenstädten anfallen dürften. Wir unterstellen daher eine Pauschale von $174 €$ durch effizienteres Parken. Die potenziellen Kostenersparnisse ergeben sich aus der Anzahl autonomer Pkw multipliziert mit dieser Pauschale:

\subsubsection{9 (Anzahl aut. Pkw bei 10\%) × $174 €$ \\ $($ Pauschale $) \approx 759,524$ Mio. $€$}

Zusätzlich soll der Zeitverlust aus der Parkplatzsuche bestimmt und monetär bewertet werden. Laut der Studie von INRIX (2017) verbringen die Deutschen jährlich rund 41 Stunden mit der Parkplatzsuche. Demnach ergäbe sich bei einem Marktanteil von $10 \%$ autonomer Fahrzeuge eine Zeitersparnis von

(4,37 Mio. (aut. Pkw bei 10\%) × 41 h (p.a.)

$\approx 179$ Mio. $\mathrm{h}$

6 Fagnant und Kockelman und auch einer der Gutachter dieses Artikels weisen darauf hin, dass den geringeren Parkkosten höhere Fahrleistungen gegenüberstehen, die gegengerechnet werden müssten. Ohne zu wissen, wohin die Parkflächen aus den Innenstädten verlagert werden, ist die sehr schwierig. Daher verzichten wir hier auf eine Gegenrechnung.
Der finanzielle Gegenwert dieser Zeitersparnis wird analog zu [4] berechnet:

(179 Mio.h (Zeitersparnis) $\times 18,1 \%$ (Ant. berufl.

Zwecke $) \times 17,125 €($ Kosten berufl. Zwecke $))$

$+(179$ Mio. $\mathrm{h}$ (Zeitersparnis) $\times(1-18,1 \%)$

$\times 7,695 €($ Kosten private Zwecke) $)$

$\approx 1,683$ Mrd. $€$

Bereits bei einem Marktanteil von nur 10\% könnten jährlich etwa 179 Millionen Stunden eingespart werden. Dies entspricht einem finanziellen Gegenwert von 1,683 Mrd. Euro. Hinzu kommen rund 759,524 Millionen Euro an direkten Einsparungen bei den Parkkosten. Die Ergebnisse werden in Tabelle 3 für alle Marktanteile zusammengefasst. Der Ansatz berücksichtigt implizit, dass nicht alle Wegstrecken mit einem Kraftfahrzeug zurückgelegt werden. Jedoch darf angenommen werden, dass der Besitz eines autonomen Fahrzeugs auch seine Nutzung bedingt. Grundlage der Berechnungen ist hier deshalb nicht die Anzahl der gültigen Fahrerlaubnisse, sondern die der autonomen Pkw.

\section{Fazit}

Die Kostenersparnisse durch Stauvermeidung, Kraftstoffeinsparungen und autonome Parkkonzepte können sowohl für alle Fahrzeugarten als auch nur für Pkw zu Gesamtsummen addiert werden, siehe Tabelle 4. Bei der gesonderten Ausweisung der Gesamtersparnisse, die allein durch private Pkw erzielt werden könnten, werden die Unfall- und Stauvermeidungspotenziale nur anteilig berücksichtigt, nämlich mit dem prozentualen Anteil autonomer Pkw am Gesamtbestand autonomer Fahrzeuge in den jeweiligen Marktanteilsszenarien. Durch den Verkehr autonomer Fahrzeuge könnten in der Bundesrepublik Deutschland bei einem Marktanteil von 50\% schätzungsweise 1.082 Leben gerettet, rund 872.500 Unfälle vermieden und jährlich fast 
24,9 Mrd. Euro eingespart werden. Diese finanzielle Gesamtersparnis setzt sich zu etwa $49 \%$ aus der Vermeidung von Unfällen, zu 11,7\% aus der Verminderung des Stauaufkommens und zu 39,3\% aus den Einsparungen der Parkkosten zusammen. Unsere Berechnungen beruhen auf einer Reihe von vereinfachenden Annahmen, die man bei der Interpretation der Ergebnisse berücksichtigen muss. Abgesehen von einer verstärkten Nutzung von Car-Sharing unterstellen wir, dass das Nutzungsverhalten von Fahrzeugen weitgehend gleichbliebe. Durch Kostensenkungen könnten aber Reboundeffekte durch eine erhöhte Nutzung von Fahrzeugen entstehen. Ebenso könnte es zu Beschäftigungs- und Einkommenseffekten in der Automobilwirtschaft oder auch der Verkehrsdienstleistungsbranche kommen, die man für eine Gesamtbetrachtung gegenrechnen müsste. Die hier angestellten Berechnungen sind nur als Ausgangspunkt für weitere Abschätzungen zu verstehen und sollten zukünftig umfangreich erweitert werden.

Zum aktuellen Zeitpunkt sind die autonomen Assistenzsysteme noch sehr kostenintensiv und stellen deshalb eine Hürde für den Durchbruch auf dem Massenmarkt dar (Fagnant und Kockelman 2015, S. 176). Laut Dellenback (2013) lagen die zusätzlichen Kosten noch vor wenigen Jahren bei über 100.000 USD pro Fahrzeug. Bei größerer Marktdurchsetzung, und dementsprechend höherer Produktionsausgabe, dürften diese jedoch mittelfristig auf bis zu 1.000 bis 3.000 USD fallen. Fagnant und Kockelman gehen davon aus, dass die zusätzlichen Kosten bei einem Marktanteil von $10 \%$ bei 10.000 , bei $50 \%$ bei 3.000 und bei $90 \%$ bei nur noch 3.000 USD liegen. Die Werte werden hier eins zu eins in die Euro-Währung übertragen. Auf Grundlage einer solchen Annahme, lässt sich die Amortisationsdauer der zusätzlichen Anschaffungskosten bestimmen. Diese liegt bei einem Marktanteil von $10 \%$ bei rund 8,3 , bei $50 \%$ bei 3,7 und bei $90 \%$ bei unter 1,7 Jahren. Die Amortisationsdauern wurden allein auf der Basis der Gesamtersparnisse durch Pkw berechnet, um die Ergebnisse aus Verbrauchersicht bewerten zu können. Einige der angesetzten Kosten, wie zum Beispiel die der Rechtsprechung oder der Polizeieinsätze, die in die Bemessung des volkswirtschaftlichen Schadens einfließen, werden von öffentlicher Hand und somit nur indirekt vom privaten Verbraucher getragen.

Eine Verringerung dieser Kosten wandelt sich also nicht unmittelbar in ein finanzielles Kaufargument für autonome Fahrzeuge um. Der größte Teil der Kosten fällt jedoch für den privaten Nutzer direkt an. Die niedrigen Amortisationsdauern lassen daher vermuten, dass für die Verbraucher ein durchaus großer finanzieller Anreiz in der Anschaffung und Nutzung autonomer Fahrzeuge besteht.

\section{Literatur}

Auto und Verkehr (2016): Parkgebühren in deutschen Städten vergleichsweise niedrig. Online verfügbar unter http://auto-und-verkehr.de/parkgebuehrendeutschen-staedten-vergleichsweise-niedrig/, zuletzt geprüft am 23.05.2018.

Barter, Paul (2013): „Cars are parked 95\% of the time“. Let's check! In: Blog Reinventing Parking. Online verfügbar unter http://www.reinventingparking. org/2013/02/cars-are-parked-95-of-time-lets-check.html, zuletzt geprüft am 23. 05.2018.

Baum, Herbert; Kranz, Thomas; Westerkamp, Ulrich (2010): Volkswirtschaftliche Kosten durch Straßenverkehrsunfälle in Deutschland. Berichte der Bundesanstalt für Straßenwesen. Mensch und Sicherheit, HeftM (208). Bremerhaven: Verlag für neue Wissenschaft.

Dellenback, Steven (2013): Director, Intelligent Systems Department, Automation and Data Systems Division, Southwest Research Institute. Communication by Email, 26. 05.2013.

Die Welt (2009): Rasende Autofahrer senken das Staurisiko. Online verfügbar unter https://www.welt.de/motor/article4252977/Rasende-Autofahrersenken-das-Staurisiko.html, zuletzt geprüft am 23. 05.2018.

Fagnant, Daniel; Kockelman, Kara (2015): Preparing a nation for autonomous vehicles. Opportunities, barriers and policy recommendations. In: Transportation Research Part A: Policy and Practice 77, S. 167-181.

HEATCO - Developing harmonised European approaches for transport costing and project assessment (2005): Deliverable 5. Proposal for harmonised guidelines. Online verfügbar unter http://heatco.ier.uni-stuttgart.de/HEATCO_ D5.pdf, zuletzt geprüft am 23.05.2018.

Kanter, Zack (2015): How Uber's autonomous cars will destroy 10 million jobs and reshape the economy by 2025. Blog. Online verfügbar unter http:// zackkanter.com/2015/01/23/how-ubers-autonomous-cars-will-destroy-10million-jobs-by-2025, zuletzt geprüft am 23. 05.2018.

Litman, Todd (2016): Parking management. Strategies, evaluation and planning. Victoria Transport Policy Institute. Online verfügbar unter: http://www.vtpi. org/park_man.pdf, zuletzt geprüft am 07.06.2018. 
Maurer, Markus; Gerdes, Christian; Lenz, Barbara; Winner, Hermann (2015): Autonomes Fahren. Technische, rechtliche und gesellschaftliche Aspekte. Berlin: Springer.

Sessa, Carlo et al. (2015): D27.2 Results on the on-line DELPHI survey. CityMobil2. Online verfügbar unter: http://www.citymobil2.eu/en/upload/Deliverables/ PU/D27\%202\%20Results\%20on\%20the\%20on-line\%20DELPHI\%20survey.pdf, zuletzt geprüft am 08.06.2018.

Shladover, Steven; Su, Dongyan; Lu, Xiao-Yun (2012): Impacts of cooperative adaptive cruise control on freeway traffic flow. In: Transportation Research Record: Journal of the Transportation Research Board (2324), S. 63-70.

Statistisches Bundesamt (2013): Verkehr auf einen Blick. Online verfügbar unter https://www.destatis.de/DE/Publikationen/Thematisch/ TransportVerkehr/Querschnitt/BroschuereVerkehrBlick0080006139004.pdf? blob=publicationFile, zuletzt geprüft am 23.05.2018.

Stern, Raphael et al. (2017): Dissipation of stop-and-go waves via control of autonomous vehicles. Field experiments. In: Transportation Research Part C: Emerging Technologies (89), S. 205-221.

strategy\& (Hg.) (2016): Connected car report 2016. Opportunities, risk, and turmoil on the road to autonomous verhicles. Online verfügbar unter https:// www.strategyand.pwc.com/media/file/Connected-car-report-2016.pdf, zuletzt geprüft am 07.06.2018.

Umweltbundesamt (2011): Klimaschutz im Stadtverkehr. Online verfügbar unter https:// www.umweltbundesamt.de/klimaschutz-im-stadtverkehr, zuletzt geprüft am 23.05.2018.

Zeit Online (2012): So parken wir morgen. Online verfügbar unter https://www. zeit.de/mobilitaet/2016-04/autonomes-fahren-parken-bosch, zuletzt geprüft am 23. 05. 2018.

\section{Forschungsdaten}

APCOA PARKING (2013): Jede Parkplatz-Suche verschlingt unnötig viel Zeit und Geld. Online verfügbar unter http://www.apcoa.de/nachrichten/extra-news/ parking-studie.html, zuletzt geprüft am 23.05.2018.

INRIX (2016): Global traffic scorecard. Ein Leitfaden zur Stausituation in Deutschland. Online verfügbar unter http://inrix.com/resources/inrix-2016-trafficscorecard-germany/, zuletzt geprüft am 23. 05.2018.

INRIX (2017): The impact of parking pain in the US, UK and Germany. Online verfügbar unter http://www2.inrix.com/research-parking-2017, zuletzt geprüft am 06.05.2018

Institut der deutschen Wirtschaft Köln (2016): Verkehrsunfälle. Anzahl. Online verfügbar unter https://www.deutschlandinzahlen.de/?621, zuletzt geprüft am 23.05.2018.

Kraftfahrt-Bundesamt (2017): Der Fahrzeugbestand im Überblick am 1. Januar 2017 gegenüber 1. Januar 2016. Online verfügbar unter https://www.kba.de/ DE/Statistik/Fahrzeuge/Bestand/Ueberblick/2017_b_ueberblick_pdf.pdf?_blob=publicationFile\&v=8, zuletzt geprüft am 23.05.2018.

Statista (2017a): Bestand an Kraftomnibussen mit Diesel-Motor in Deutschland von 1996 bis 2017 (in 1.000 Stück). Online verfügbar unter https://de.statista. com/statistik/daten/studie/251774/umfrage/bestand-an-kraftomnibussenmit-diesel-motor-in-deutschland/, zuletzt geprüft am 23.05.2018.

Statista (2017 b): Bestand an Lkw mit Diesel-Motoren in Deutschland von 1998 bis 2017 (in 1.000 Stück). Online verfügbar unter https://de.statista.com/ statistik/daten/studie/251786/umfrage/bestand-an-lkw-mit-diesel-motorin-deutschland/, zuletzt geprüft am 23.05.2018.

Statista (2017 c): Durchschnittlicher Benzinpreis in Deutschland in den Jahren
1972 bis 2017 (Cent pro Liter Superbenzin). Online verfügbar unter https:// de.statista.com/statistik/daten/studie/776/umfrage/durchschnittspreis-fuersuperbenzin-seit-dem-jahr-1972/, zuletzt geprüft am 23. 05.2018.

Statista (2017 d): Durchschnittlicher Preis für Dieselkraftstoff in Deutschland in den Jahren 1950 bis 2017* (Cent pro Liter). Online verfügbar unter https:// de.statista.com/statistik/daten/studie/779/umfrage/durchschnittspreis-fuerdieselkraftstoff-seit-dem-jahr-1950/, zuletzt geprüft am 23. 05.2018.

Statista (2017 e): Statistiken zum Thema Autofahrer. Online verfügbar unter https://de.statista.com/themen/759/autofahrer/, zuletzt geprüft am 23.05.2018.

Statistisches Bundesamt (2017) 2016. Mehr Unfälle, aber weniger Verkehrstote als jemals zuvor. Online verfügbar unter https://www.destatis.de/DE/ PresseService/Presse/Pressemitteilungen/2017/07/PD17_230_46241pdf.pdf?_blob=publicationFile, zuletzt geprüft am 23.05.2018.

Umweltbundesamt (2012): Daten zum Verkehr. Online verfügbar unter https:// www.umweltbundesamt.de/sites/default/files/medien/publikation/ long/4364.pdf, zuletzt geprüft am 23.05.2018.

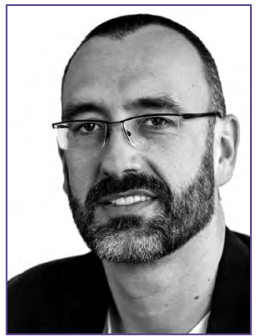

PROF. DR. MICHAEL ROOS

ist seit 2009 Inhaber des Lehrstuhls für Makroökonomik an der Ruhr-Universität Bochum und arbeitet vor allem in den Bereichen Komplexitätsökonomik, agentenbasierte Modellierung, Nachhaltigkeit und ökonomische Methodologie.

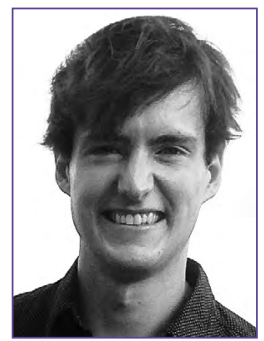

\section{MARVIN SIEGMANN}

hat einen Bachelor of Science, studiert Ethics Economics, Law and Politics im Masterprogramm der Ruhr-Universität Bochum und arbeitet am Lehrstuhl für Makroökonomik in den Bereichen automatisiertes Fahren und agentenbasierte Modellierung. 


\section{Automatisiertes Fahren als Herausforderung für Städte und Regionen}

Eine interdisziplinäre Analyse denkbarer Anwendungen und Effekte

Fabienne Perret, EBP Schweiz AG, Geschäftsbereich Verkehr, Mühlebachstrasse 11, 8032 Zürich (fabienne.perret@ebp.ch)

Remo Fischer, EBP Schweiz AG, Geschäftsbereich Verkehr, Zürich (remo.fischer@ebp.ch)

Holger Frantz, EBP Deutschland GmbH, Berlin (holger.frantz@ebp.de)

Das automatisierte Fahren hat das Potenzial, unsere alltägliche Mobilität grundlegend zu verändern. Auf Basis eines denkbaren Entwicklungspfades werden die heute absehbaren Auswirkungen, insbesondere aus Sicht der Städte und Regionen, hergeleitet. Dabei stehen Chancen wie Effizienzgewinne, neue Mobilitätsangebote und eine Erhöhung der Verkehrssicherheit den Herausforderungen wie potenzielle Verkehrszunahmen, Anpassungen der Regulierungen oder der Aufbau einer Dateninfrastruktur gegenüber. Ob das automatisierte Fahren gesamthaft einen Nutzen bringen wird, hängt deutlich von der Einflussnahme der Politik ab.

\section{Automated driving as a challenge to cities and regions} An interdisciplinary analysis of potential applications and effects

Automated driving has the potential to fundamentally change our everyday mobility. Based on a conceivable development path, the effects that are foreseeable from today's perspective of cities and regions are described. Automated vehicles offer numerous opportunities such as efficiency gains, new mobility options, and increased safety. Upcoming challenges are potential traffic growth, necessary adjustments in legislation, and the development of an adequate data infrastructure. The overall benefit of automated driving strongly depends on political influence.

Keywords: automated vehicles, driverless cars, sharing services, public transportation, traffic management

This is an article distributed under the terms of the Creative Commons Attribution License CCBY 4.0 (https://creativecommons.org/licenses/by/4.0/)

https://doi.org/10.14512/tatup.27.2.31

Submitted: 09. 03.2018. Peer reviewed. Accepted: 11. 05.2018

\section{Hintergrund}

Das Thema automatisiertes Fahren wurde bisher vor allem durch Technologieunternehmen und Industrieunternehmen besetzt, die es primär mit Vernetzung, Sharing und Elektromobilität in Verbindung gebracht haben - nicht zuletzt, um sich aus Gründen des Marketings als Innovationsträger zu präsentieren. Gesichertes Wissen über die Wirkungen des automatisierten Fahrens auf das Mobilitätsverhalten, auf das gesamte Verkehrsgeschehen, auf die Raumentwicklung oder den Infrastrukturbedarf fehlt jedoch weitgehend. Diese Aspekte finden aktuell vermehrt Eingang in Forschungsbestrebungen.

Neben den Hochleistungsstraßen, die in besonderem Maße im Fokus der Bundesregierungen liegen (Deutsche Bundesregierung 2015; Schweizerischer Bundesrat 2016), stellen sich für die Planerinnen und Planer in den Städten und Regionen zentrale Fragen zum untergeordneten Verkehrsnetz: Welchen Einfluss haben automatisierte Fahrzeuge auf die Kapazität der Verkehrsnetze und das Erscheinungsbild der Mobilität im Siedlungsgebiet? Bedarf es neuer Infrastrukturen? Welches sind die Rollen und Verantwortlichkeiten der Kommunen und Städte? Wem gehören die produzierten Daten? Welche Mobilitätsangebote haben Marktchancen?

\section{Vorgehen}

Im Rahmen einer interdisziplinären Studie (EBP 2017), welche in Zusammenarbeit mit zahlreichen schweizerischen Akteuren (u. a. Städte, Kantone, Transportunternehmen) entstand, wurden die relevanten Aspekte des automatisierten Fahrens diskutiert und mögliche Auswirkungen abgeschätzt. Der gemeinsam festgelegte Fokus lag auf planerischen Aspekten des Personenverkehrs auf Schiene und Straße, rechtliche Fragen standen nicht im Zentrum. 
Das methodische Vorgehen umfasste zuerst eine Literaturanalyse zu den Definitionen und Wirkungsmechanismen. In interdisziplinären Workshops wurden mit den beteiligten Akteuren der Studie ausgewählte Fragestellungen vertieft und insbesondere der Entwicklungspfad erarbeitet. Auf dieser Grundlage konnten weitere Literaturanalysen zu ausgewählten Fragestellungen, quantitative Abschätzungen sowie gezielte Experteninterviews zur Vertiefung durchgeführt werden.

\section{Denkbarer Entwicklungspfad}

Für die Untersuchung wurde angenommen, dass die menschliche Innovationskraft das automatisierte Fahren früher oder später erfolgreich ermöglichen wird. Die Gesellschaft wird das automatisierte Fahren in der Zukunft als alltäglich betrachten und breit nutzen. Doch wie kommen wir dahin? Um die Auswirkungen über die Zeit beschreiben zu können, wurde für die Schweiz ein Entwicklungspfad mit sechs Zuständen skizziert. Er wird aus heutiger Sicht sowohl von den involvierten Akteuren als auch von den Autorinnen und Autoren als plausibel und wahrscheinlich eingeschätzt. Es wird dementsprechend nicht der Anspruch erhoben, dass dieser die einzig mögliche Entwicklung darstellt. Welche Abfolge tatsachlich eintreten wird, ist von vielen Faktoren abhängig. Der skizzierte Entwicklungspfad ermöglicht es jedoch, die Komplexität für Wirkungsanalysen zu reduzieren und die Handlungsoptionen in Schritten aufzuzeigen.

Grundsätzlich kann der Entwicklungspfad auf Deutschland adaptiert werden, allerdings müssen die unterschiedliche Bedeutung der Automobilindustrie (zahlreiche Automobilhersteller in Deutschland) und des öffentlichen Verkehrs (tieferer Nachfrageanteil in Deutschland als in der Schweiz) berücksichtigt werden. Der Pfad zeigt einen plausiblen Verlauf der Entwicklung in unterschiedlichen Teilräumen auf, er beinhaltet jedoch keine konkreten Zeithorizonte. Tabelle 1 gibt einen Überblick über die erarbeiteten sechs Zustände des Entwicklungspfads der Automatisierung nach Anwendungsbereichen.

\section{Motorisierter Individualverkehr}

Abbildung 1 zeigt die sechs Zustände des Entwicklungspfads der Automatisierung im motorisierten Individualverkehr (MIV). Dabei werden die internationalen Automatisierungsgrade (levels of automation) verwendet (SAE 2014). Der MIV dürfte eine schrittweise generische Entwicklung vom konventionellen selbstgesteuerten Fahrzeug bis hin zur vollständigen Automatisierung durchlaufen. Die nötigen Bewilligungen bzw. Zulassungen werden technisch und räumlich ausgeweitet - von der Sonderbewilligung für Teststrecken an einzelne Entwickler über die temporäre Freigabe von Pilotstrecken für Nutzer mit entsprechend zugelassenem Fahrzeug, hin zur allgemeinen Freigabe im gesamten Straßennetz.

Automatisierte Fahrzeuge dürften sich zuerst auf den Hochleistungsstraßen (Zustände 2 und 3 gemäß Abb. 1) und dann im städtischen Raum (Zustände 3 und 4 gemäß Abb. 1) durchsetzen. Der Betrieb des Autobahnnetzes als geschlossenes System ist einfacher: Die Fahrtrichtungen sind baulich getrennt, Konflikte der Trajektorien kommen nur in Bezug auf Spurwechselvorgänge vor und es sind keine anderen Verkehrsmittel vorhanden. In Siedlungsräumen ist die Situation aufgrund des Mischverkehrs mit anderen Verkehrsmitteln und den zahlreichen Fahrkonflikten zwar deutlich komplexer als außerorts. Die gefahrenen Geschwindigkeiten sind jedoch niedriger und damit die Möglichkeiten der Erfassungstechnologien in Bezug auf die Erkennung von sich bewegenden Objekten deutlich besser. Darüber hinaus dürfte die Datennetzabdeckung in Siedlungsräumen über lange Zeit besser sein als in ländlichen Gebieten. Deshalb wird gemäß Abbildung 1 erst ab Zustand 4 mit einer Anwendung des automatisierten Fahrens auf Überlandstraßen gerechnet.

\begin{tabular}{|c|c|c|c|}
\hline Zustand & Motorisierter Individualverkehr (MIV) & Straßengebundener ÖPNV & Schienenverkehr \\
\hline 1 & Fahrassistenzsysteme L1/L2 auf Gesamtnetz & Teststrecken (L4) & Isolierte Anwendungen (GoA 3/4) \\
\hline 2 & Freigabe L3 auf Hochleistungsstraßen & $\begin{array}{l}\text { Automatisierte Kleinbusse innerorts im Regel- } \\
\text { betrieb (L4) }\end{array}$ & Ausgewählte Teststrecken (GoA 3/4) \\
\hline 3 & $\begin{array}{l}\text { Freigabe L4 auf Hochleistungsstraßen, } \\
\text { L3 auf Straßen im Siedlungsraum }\end{array}$ & $\begin{array}{l}\text { Automatisierte Kleinbusse innerorts im Regel- } \\
\text { betrieb (L5) }\end{array}$ & \multirow[t]{2}{*}{$\begin{array}{l}\text { Automatisierter Zugbetrieb in Teilnetzen } \\
\text { (GoA 3/4) }\end{array}$} \\
\hline 4 & $\begin{array}{l}\text { Freigabe L4 auf Straßen im Siedlungsraum, } \\
\text { L3 auf Überlandstraßen }\end{array}$ & $\begin{array}{l}\text { Automatisierte Kleinbusse inner-/außerorts } \\
\text { im Regelbetrieb (L5) }\end{array}$ & \\
\hline 5 & $\begin{array}{l}\text { L4 auf Überlandstraßen und schließlich } \\
\text { L5 auf dem Gesamtnetz }\end{array}$ & $\begin{array}{l}\text { Alle Fahrzeuge inner-/außerorts im Regel- } \\
\text { betrieb (L5), adaptiver/linienfreier ÖPNV } \\
\text { (insb. auf nachfrageschwachen Beziehungen) }\end{array}$ & \multirow[t]{2}{*}{$\begin{array}{l}\text { Automatisierter Zugbetrieb im Gesamtnetz } \\
(\text { GoA 3/4) }\end{array}$} \\
\hline 6 & $\begin{array}{l}\text { L5 auf dem Gesamtnetz, hoheitliche Einfluss- } \\
\text { nahme }\end{array}$ & Zusätzlich: Hoheitliche Einflussnahme & \\
\hline
\end{tabular}

Tab. 1: Kurzfassung der Entwicklungspfade über sechs Zustände hinweg für die drei genannten Anwendungsbereiche. Level (L) bzw. Grade of Automation (GoA) geben die Automatisierungsstufen gemäß SAE- bzw. UITP-Standards an. 


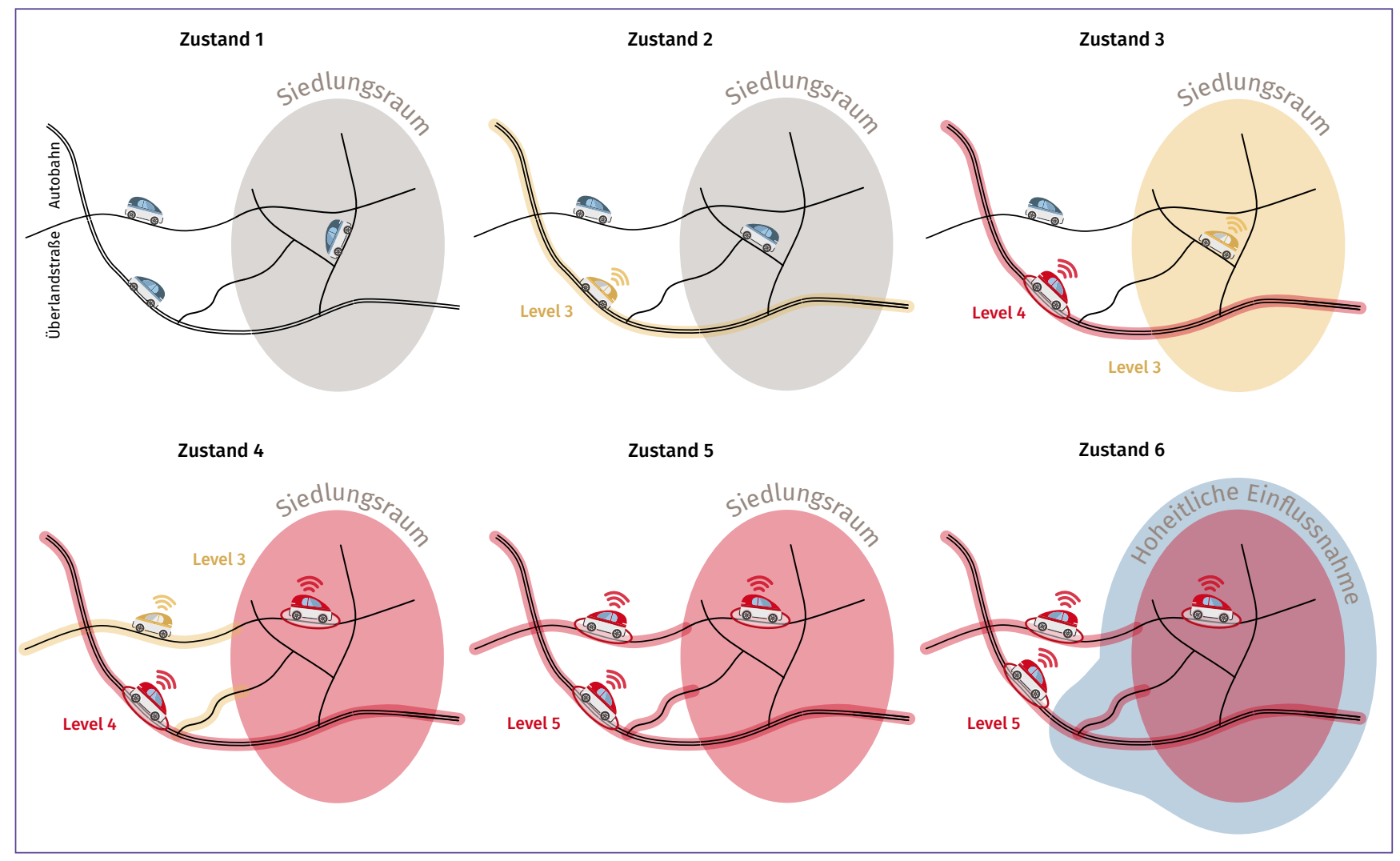

Abb.1: Entwicklungspfad beim MIV (Zustände 1-6).

Quelle: Eigene Darstellung

Mit zunehmender Automatisierung wird das Mobilsein attraktiver, unter anderem weil die Reisezeit im Auto für andere Aktivitäten genutzt werden kann. Dies dürfte die Verkehrsnachfrage deutlich steigern, wie die detaillierte Auswirkungsanalyse im Abschnitt „Auswirkungen“ aufzeigt. Da der Spielraum für einen Ausbau der Verkehrsinfrastrukturen in der Schweiz sehr begrenzt ist und eine ungebremste Zunahme des Verkehrsaufkommens politisch nicht erwünscht ist, drängen sich Lenkungsinstrumente auf. Auf Basis einer Iteration wurde daher im letzten Zustand des Entwicklungspfades (Zustand 6 gemäß Abb. 1) von einer staatlichen Einflussnahme ausgegangen, welche das Mobilitätsverhalten mit (neuen) Regulativen und Anreizsystemen in eine nachhaltige Richtung steuert. Davon können alle Verkehrsmittel betroffen sein. Zur Einflussnahme gehören beispielsweise Anreize zur Bündelung von Wegen oder die Beschränkung von Leerfahrten. Die Anwendung solcher Instrumente kann auch schon in früheren Zuständen erfolgen, wurde hier aber zur differenzierten Folgenabschätzung bewusst an das Ende des Entwicklungspfades gestellt.

\section{Straßengebundener ÖPNV}

Beim straßengebundenen öffentlichen Personennahverkehr (ÖPNV) bieten sich infolge der Automatisierung Möglichkeiten für veränderte Transportangebote (UITP 2017). Bereits heute werden in zahlreichen Städten automatisierte Minibusse mit Sonderbewilligungen und unter Begleitung getestet. Anwendungen dürften sich über den automatisierten Betrieb einzelner Linien, dem automatisierten Regelbetrieb im ÖPNV-Netz, hin zu einem adaptivem ÖPNV entwickeln. Dieser folgt keiner definierten Linienführung mehr, sondern führt bei jeder Fahrt ein optimiertes Routing zur Bündelung der angemeldeten Fahrtenwünsche der Kunden durch. Insbesondere in peripheren Stadtgebieten und im ländlichen Raum weist diese Angebotsform Vorteile auf (kostengünstige, gute Bedienung bei geringer Nachfrage). In dichten städtischen Räumen dürften auf Hauptachsen weiterhin große Fahrzeuggefäße im Linienverkehr zum Einsatz kommen, da sie in Bezug auf Kapazitäten und Bündelung kaum zu ersetzen sind.

\section{Car-Sharing und Pooling}

Im Übergangsbereich von MIV und ÖPNV besteht infolge des führerlosen Betriebs (mind. SAE-Level 4) das Potenzial für neue Verkehrsangebote. Dazu gehört einerseits das Car-Sharing, bei dem Fahrzeuge einer automatisierten Flotte individuell und seriell genutzt werden können. Andererseits werden auch Pooling-Angebote in der Form von automatisierten Sammeltaxis möglich, bei denen Fahrzeuge von verschiedenen Personen gleichzeitig genutzt werden und so ein Bündelungseffekt eintritt. Diese Sammeltaxis optimieren ihren Betrieb auf der Basis von angemeldeten Fahrten (on-demand). Sie können in den ÖPNV 


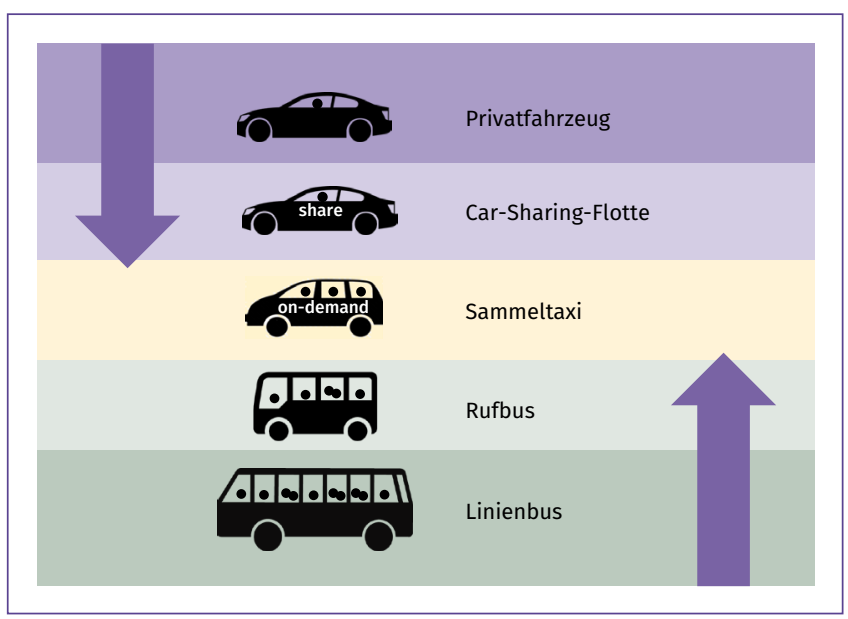

Abb.2: Angebotsformen zwischen MIV und ÖPNV.

Quelle: Eigene Darstellung

eingebunden sein oder von privaten Anbietern betrieben werden. Intensives Car-Sharing und Pooling bedingen einen Bedeutungsverlust des individuellen Fahrzeugbesitzes. Abbildung 2 gibt die beschriebenen Angebotsformen wieder, ohne auf die Abhängigkeiten von Gesamtflotten, Fahrzeuggröße, Fahrzeugbetrieb oder Routingprinzipien einzugehen. Diese sind Bestandteil von anderen Forschungsarbeiten (z. B. OECD/ITF 2015).

\section{Schienengebundener Öffentlicher Verkehr (ÖV)}

Auch für den schienengebundenen ÖV wurde im Rahmen der Studie ein Entwicklungspfad erarbeitet, der von bereits heute vorhandenen netztopologisch isolierten Anwendungen (z. B. führerlose U-Bahnen) hin zu einem automatisierten, führerlosen Zugbetrieb im gesamten Schienennetz führt. Die Abfolge der Zustände wird dabei als weniger komplex als im Straßenverkehr angenommen, da die Fahrzeuge fixe Strecken befahren, heute schon in übergeordnete Steuerungen eingebunden sind und als Flotten beschafft sowie erneuert werden. Wie rasch sich führerloses Fahren auf der Schiene durchsetzt, ist aber insbesondere auch von Akzeptanzfragen bei den Nutzenden und von den langlebigen Fahrzeuggenerationen abhängig. Das ÖV-Rollmaterial ist deutlich länger im Einsatz als im Individualverkehr.

\section{Auswirkungen}

\section{Faktoren für die Marktdurchdringung automatisierter Fahrzeuge}

Im Hinblick auf eine Abschätzung der verkehrlichen Wirkungen muss analysiert werden, wie sich automatisierte Fahrzeuge in der Gesamtflotte über die Zustände verbreiten werden. Dabei sind insbesondere die individuellen Zusatznutzen als auch die Zusatzkosten entscheidend. Zusatznutzen entstehen für die Käufer hinsichtlich der Sicherheit. In den ersten Zuständen geht das automatisierte Fahren vor allem mit einer höheren passiven Sicherheit einher, da Eingriffe in Längs- und Querführung des
Fahrzeuges das Ausmaß und die Folgen der Unfälle reduzieren. In einer zweiten Phase mit zunehmender Vernetzung zwischen den Fahrzeugen wird durch die weitgehende Vermeidung von Unfällen auch die aktive Sicherheit erhöht. Ein weiterer wichtiger Nutzen entsteht durch den Zeitgewinn. In den ersten $\mathrm{Zu}-$ ständen des automatisierten Fahrens kann ein Teil der Unterwegszeit für alternative Tätigkeiten genutzt werden. In den $\mathrm{Zu}-$ ständen mit Vollautomatisierung können Fahrzeuge führerlos unterwegs sein, was den Reisekomfort und damit den Nutzen weiter erhöht und eine zeitliche Flexibilität dank der Möglichkeit von Fahraufträgen bringt. Die Zusatzkosten sind vor allem durch die erhöhte technische Ausstattung der Fahrzeuge sowie die zunehmenden Kommunikationskosten (Datenübertragung in Echtzeit) begründet.

Bei der Abschätzung der Marktdurchdringung ist zu berücksichtigen, dass auch künftig ein Unterschied zwischen den technischen Möglichkeiten und den gesetzlich erlaubten Funktionen bestehen wird. Fahrzeuge werden voraussichtlich bis zur Erreichung von Zustand 4 mehr können als sie dürfen. Das heißt im Umkehrschluss: Nur, weil die technologischen Voraussetzungen gegeben sind, kann nicht auch mit einer raschen Verbreitung des automatisierten Fahrens gerechnet werden - weder im ÖV noch beim MIV. Der Unterschied ist im heutigen Zustand 1 noch bescheiden, dürfte jedoch in den Zuständen 2 und 3 recht erheblich sein (Abb. 3).

\section{Einfluss auf die Verkehrsnachfrage}

Automatisierte Fahrzeuge verändern das gesamte Verkehrssystem und damit die verkehrliche Nachfrage. Relevante Einflussfaktoren auf die Nachfrage wurden identifiziert und im Zusammenhang mit automatisiertem Fahren abgeschätzt.

Nutzung der Reisezeit: Durch die Automatisierung wird die Reisezeit anderweitig nutzbar. In den ersten Zuständen muss der Fahrer zur Übernahme der Fahraufgaben bereit sein (beispielsweise in Zustand 2 auf Hochleistungsstraßen), in den weiteren Zuständen ist diese Bereitschaft nicht mehr notwendig. Durch die Nutzung der Reisezeit sinken die individuellen Reisezeitkosten, weshalb allenfalls weiter entfernt gelegene Ziele angefahren werden. Reisedauer und Reiseweiten könnten sich erhöhen.

Neue Nutzergruppen: Beim automatisierten Fahren werden Fahraufgaben vom Fahrer an das System abgegeben, bei tieferen Automatisierungsstufen temporär und bei höheren Levels dauerhaft. In den höchsten Automatisierungsstufen kann das Steuern des Fahrzeuges durch den Menschen gänzlich entfallen. Damit werden die Anforderungen an den Fahrer gesenkt bzw. aufgehoben. Auch bisher mobilitätseingeschränkte Personen können Fahrzeuge ohne Führerschein und ohne Begleitung eines Fahrers nutzen. Dies betrifft insbesondere Kinder, Jugendliche und Senioren.

Leerfahrten: Sind Fahrzeuge vollautomatisiert unterwegs, muss kein Fahrer mehr an Bord sein. Vor allem bei der privaten Nutzung dürften so durch das Bringen und Abholen von Personen und Waren viele Leerfahrten entstehen. Aber auch bei automatisierten Sharing-Fahrzeugen müssten Fahrten ohne Pas- 
sagiere zwischen den Ab- und Zugangsorten durchgeführt werden. Entstehende Leerfahrten erhöhen die Fahrleistung und reduzieren den durchschnittlichen Besetzungsgrad.

Änderung der Verkehrsmittelwahl: Die Abschätzung der Auswirkungen auf den Modal Split ist am unsichersten, weil gegenläufige Effekte eintreten könnten. Im Zuge der Automatisierung werden sich die verkehrlichen Angebote in ihren Vor- und Nachteilen verändern. Die Verkehrsmittelwahl ist vor allem abhängig von den Preisen, den Reisezeiten und dem Komfort. Durch den Entfall des Fah-

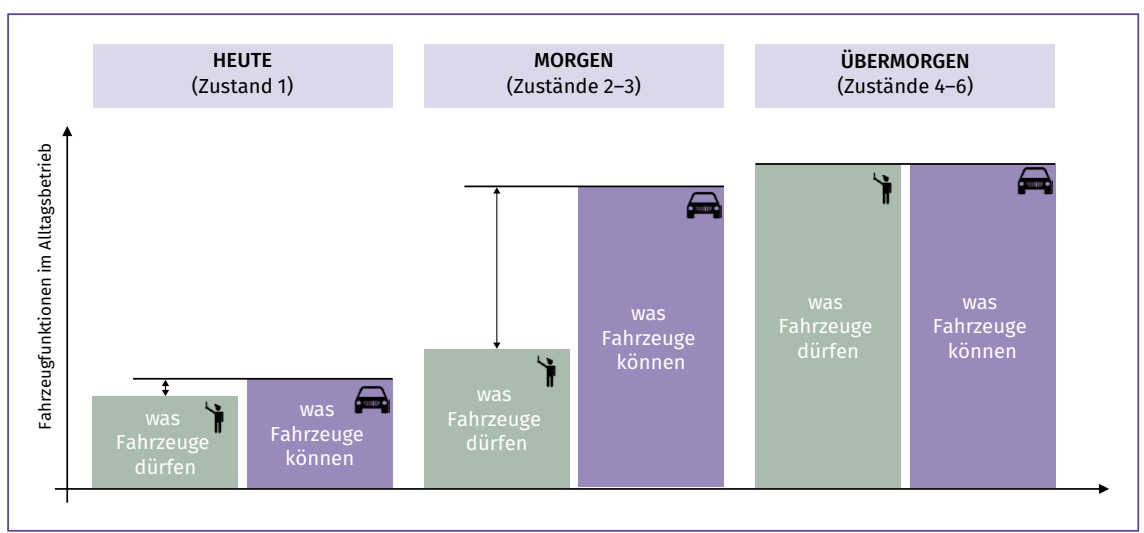

Abb.3: Diskrepanz zwischen den technologischen Möglichkeiten und der zugelassenen Nutzung.

Quelle: Eigene Darstellung bei privaten Sharing-Diensten deutliche

Kostenvorteile erreicht (Bösch et al. 2017). Infolge von tieferen Preisen gewinnen diese Angebote an Attraktivität. Beim MIV sind aber nicht die Gesamtkosten, sondern die variablen Kosten (z. B. Treibstoff) für die unmittelbare Verkehrsmittelwahl maßgebend. Und diese dürften weiterhin geringer ausfallen als die Preise der neuen Angebote (Bösch et al. 2017). Privatfahrzeuge weisen überdies einen höheren Komfort auf. Trotzdem dürfte vor allem der MIV in Bezug auf die Verkehrsleistung Anteile an die neuen automatisierten Angebote verlieren. Beim ÖV bestehen durch die Kosteneinsparungen im Zuge der Automatisierung (Entfall von Personal) verschiedene nachfragesteigernde Möglichkeiten: Bei gleich hohen Abgeltungsleistungen und Tarifen können mehr Leistungen bestellt und das Angebot somit ausgeweitet werden. Bei gleichbleibenden Abgeltungen und gleichem Angebot können Tarife gesenkt werden, was Umsteiger generiert.

Kapazitäten und Reisezeiten: Durch die Automatisierung kann die Kapazität der bestehenden Infrastruktur vergrößert werden, da geringere mittlere Folgezeitlücken zwischen automatisierten Fahrzeugen erreicht werden können. Simulationsresultate für Hochleistungsstraßen zeigen unter der Berücksichtigung von unterschiedlichen Streckenabschnitten, Durchdringungsraten automatisierter Fahrzeuge, Geschwindigkeitsregimes, Schwerverkehrsanteilen sowie Verkehrsstromverhältnissen Kapazitätswirkungen in der Größenordnung von 0 bis zu 30\% (Fellendorf 2017). Allerdings sind die Angaben zu Zeitlücken, die automatisierte Fahrzeuge einhalten können, noch nicht gesichert und von Annahmen zur Vernetzung der Fahrzeuge abhängig. Wenn automatisierte Fahrzeuge in den früheren Zuständen aufgrund von Sicherheitsüberlegungen höhere mittlere Zeitlücken einhalten, als dies heute der Fall ist, würde beispielsweise auf dem Autobahnnetz in Deutschland die Kapazität vorerst abnehmen (Hartmann et al. 2017). In Städten besteht bei vollständiger Durchdringung ein theoretisches Potenzial für Kapazitätssteigerungen von rund 20 bis $40 \%$ (Friedrich 2015). Dabei sind vor allem Knoten mit Lichtsignalanlagen maßgebend, an denen ein verringerter Zeitbedarf pro Fahrzeug, eine Harmonisierung der Zeitlücken, ein unverzögertes Anfahren sowie eine erhöhte Räumgeschwindigkeit infolge der Automatisierung erwartet werden. Aufgrund von zusätzlich zu berücksichtigenden Interaktionen unter den Verkehrsteilnehmenden dürfte die Kapazitätswirkung in der Stadt aber kleiner sein als auf dem Hochleistungsnetz. Die Kapazitätszunahmen können zu Mehrverkehr auf der Straße führen (neu generierte Fahrten, veränderte Zielwahl, Verkehrsträgerverlagerungen). Als Folge verändern sich Reisezeiten und Erreichbarkeiten im Verkehrssystem.

\section{Infrastruktur}

Automatisierte Fahrzeuge sind auf eine adäquate Datenübertragungsinfrastruktur angewiesen, damit die Potenziale für einen optimalen Betrieb der Verkehrsinfrastruktur genutzt werden können. Hierzu sollen Fahrzeuge untereinander und mit der Infrastruktur kommunizieren. Beispielsweise können so die Zeitlücken zwischen den Fahrzeugen weiter reduziert und die Kapazitäten erhöht werden (Hartmann et al. 2017). Zudem besteht die Möglichkeit, dass Ereignisdaten ausgetauscht werden, insbesondere zu Gefahren oder Stauzuständen auf dem Netz, oder dass das Verkehrsmanagement mit geeigneten Instrumenten erweitert wird, damit sich das Verkehrssystem einem Systemoptimum annähert. Voraussetzung, um diesen Nutzen zu realisieren, ist die Anpassung der digitalen Infrastruktur.

Bei den Straßenkapazitäten ist zwar eine Erhöhung infolge der Automatisierung möglich, diese steigen allerdings erst mit einer hohen Durchdringung automatisierter Fahrzeuge in relevantem Ausmaß an (Friedrich 2015). Damit zeichnen sich zumindest mittelfristig keine deutlichen Sprünge ab. Zudem ist bei der Leistungsfähigkeit immer der Abschnitt mit der geringsten Kapazität maßgebend, daher muss immer eine Netzbetrachtung erfolgen. Die Kapazitätseffekte können allenfalls punktuell helfen, den Bedarf an Infrastrukturausbauten zu reduzieren.

Im Siedlungsraum stehen Veränderungen bei der Verkehrsinfrastruktur an: Infolge der zunehmenden Nutzung von automatisierten Sharing-/Pooling-Fahrzeugen müssen genügend Haltemöglichkeiten definiert und rechtlich geregelt werden. Gleichzeitig könnte der Bedarf an Parkierungsflächen deutlich reduziert 
werden, wenn kollektive Angebote intensiv benutzt werden und auf das Privatfahrzeug verzichtet wird. Zu klären sind zusätzlich die Anforderungen an Straßenmarkierungen und Verkehrssignale.

Das Nebeneinander verschiedener Verkehrsmittel nimmt insbesondere im Siedlungsgebiet zu: Es wird nicht mehr nur zwischen Fußverkehr, Radverkehr, ÖPNV und MIV mit ihren Ansprüchen an den Verkehrsraum zu unterscheiden sein. Einerseits werden neue Mischformen zwischen ÖPNV und MIV auftreten, die weitere Ansprüche an den öffentlichen Raum mitbringen. Andererseits ist beim motorisierten Verkehr auch zwischen den verschiedenen Automatisierungsgraden, deren Anforderungen sowie deren Vorschriften und Rahmenbedingungen zu unterscheiden. Es ist davon auszugehen, dass Fahrzeuge während langer Zeiträume auf unterschiedlichen Automatisierungsstufen im gleichen Raum unterwegs sind. Diese Mischverkehre dürften unseren Alltag in den kommenden Jahrzehnten prägen und sich auf das Erscheinungsbild der Mobilität in Siedlungsräumen auswirken. Die innerstädtischen Straßenräume müssen an die Anforderungen dieses Mischverkehrs zwischen automatisierten und nicht automatisierten Fahrzeugen sowie weiteren Verkehrsteilnehmenden, insbesondere dem Fuß- und Radverkehr, angepasst werden.

\section{Kollektiver Personenverkehr}

Der straßengebundene ÖPNV erhält durch die Vollautomatisierung neue Perspektiven. Längerfristig besteht die Möglichkeit, die Mobilität nachfrageorientierter zu gestalten. In nachfrageschwachen Gebieten können Linien aufgehoben und das Routing adaptiv auf die Kundenwünsche gestaltet werden. Durch selbstfahrende Fahrzeuge ergeben sich im Bereich des Sharings und Poolings neue Möglichkeiten zur Organisation der Transportketten. Automatisierte Fahrzeuge können durch entsprechende Weg-Anmeldungen kollektiv oder individuell verwendet werden. Mit der Automatisierung verschärft sich der Wettbewerb zwischen den verschiedenen Verkehrsmitteln. Eine Veränderung des Modal Splits ist die wahrscheinliche Folge.

Gefragt sind auch intermodale Plattformen, welche die öffentlichen und privaten Transportleistungen kombinieren sowie Wegeketten mit verschiedenen Verkehrsmitteln organisieren und die Bezahlung über ein einziges Konto regeln. Für öffentliche Transportunternehmen stellt sich damit bereits heute die Frage, inwiefern sie die Chancen solcher Mobilitätsdienste (Mobilityas-a-Service) nutzen können.

\section{Fazit und Handlungsbedarf}

\section{Chancen und Herausforderungen}

Aus Sicht der Städte und Regionen bietet das automatisierte Fahren zahlreiche Chancen: Pooling- und Sharing-Fahrzeuge führen zu Effizienz- und Flächengewinnen beim Parkraum. Bei einer Reduktion von Folgezeitlücken sind Kapazitätsgewinne und eine erhöhte Stabilität des Verkehrsflusses auf der Straße möglich. Bei hoher Durchdringung mit automatisierten Fahrzeugen können allenfalls Knotensteuerungen angepasst werden. Durch das Vermeiden menschlicher Fehler könnte die Verkehrssicherheit erhöht werden. Automatisierte Fahrzeuge ermöglichen eine verbesserte, auf einer Datenkommunikation basierte Verkehrssteuerung. Mobility-as-a-Service-Angebote können die individuelle Mobilität erleichtern. Neue Angebote im ÖPNV und Sharing-Fahrzeuge verbessern die Erschließungsqualität und ermöglichen Mobilität für bisher mobilitätseingeschränkte Nutzergruppen.

Die Herausforderungen bestehen vor allem in der potenziellen Zunahme der verkehrlichen Nachfrage und der damit verbundenen Erhöhung der Fahrleistung. Verschiedene treibende Nachfrageeffekte überlagern sich: Mit der (Voll-)Automatisierung sind Leerfahrten möglich, es entstehen neue Transportangebote, und ohne die Notwendigkeit eines Fahrers werden auch Nutzer ohne klassischem Führerschein in automatisierten Fahrzeugen unterwegs sein können. Kapazitätserhöhungen infolge der Automatisierung führen zu Mehrverkehr. Zudem könnten diese die Erreichbarkeiten so verändern, dass insbesondere ländliche Gemeinden und Agglomerationen profitieren, während Städte an Attraktivität einbüßen (Meyer et al. 2016). Folge davon ist eine Zunahme der Zersiedelung. Was also müssen die Behörden auf allen Stufen tun und wie müssen sie sich abstimmen, um Chancen zu nutzen und Herausforderungen anzugehen (Abb.4)? Wie können sich ÖPNV-Anbieter und Autohersteller positionieren?

\section{Mobilitäts- und Verkehrsmanagement}

Behörden können mit verschiedenen verkehrsplanerischen, -technischen und -politischen Maßnahmen lenkend eingreifen und mögliche Fehlentwicklungen korrigieren. Insbesondere müssen Instrumente gefunden werden, welche die potenzielle Erhöhung der Fahrleistungen infolge der nutzbaren Reisezeiten abschwächen sowie das Ausmaß von Leerfahrten automatisierter Fahrzeuge beeinflussen. Leerfahrten von neuen Sharing- und Pooling-Angeboten dürften kaum zu verhindern sein, sie haben jedoch - insbesondere im MIV - das Potenzial, weitere Verkehrszusammenbrüche zu provozieren. Dabei sind Zufahrtsbeschränkungen für leere Fahrzeuge, ein Mobility Pricing oder Subventionen für Pooling denkbar. Weiter könnte zum Beispiel in Zonen, wo ein hohes Verkehrsaufkommen regelmäßig zu Staus führt, der Einsatz von vollautomatisierten Fahrzeugsystemen für entsprechend ausgerüstete Fahrzeuge zur Pflicht werden. Auch muss die kollektive Fahrzeugnutzung mit geeigneten Anreizen

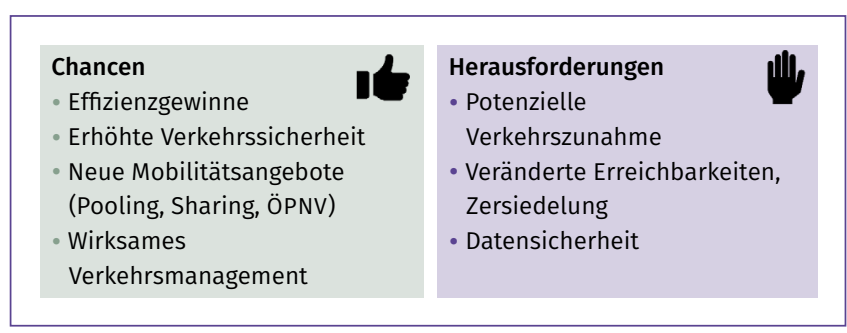

Abb.4: Chancen und Herausforderungen des automatisierten Fahrens. Quelle: Eigene Darstellung 
gefördert werden. Die staatlichen Abgeltungen im ÖV müssen auf die neue Konkurrenzsituation von Verkehrsmitteln ausgerichtet werden. In welchem Ausmaß solche Maßnahmen im Mobilitätsund Verkehrsmanagement von der Gesellschaft akzeptiert und umgesetzt werden können, wird letztlich von der jeweiligen nationalen, regionalen und kommunalen Verkehrspolitik abhängig sein und sich erst noch zeigen müssen.

\section{Handlungsbedarf}

Die Auseinandersetzung mit dem Entwicklungspfad führt zu ganz unterschiedlichen Erkenntnissen. Bereits die der Studie zugrunde gelegten Annahmen zeigen, wie vielfältig die Zukunft des automatisierten Fahrens tatsächlich aussehen könnte. Letztlich müssen wir als Gesellschaft diskutieren, welche Vorteile des automatisierten Fahrens wir nutzen und welche unerwünschten Entwicklungen wir vermeiden wollen. Die Vorteile von automatisierten Fahrzeugen wie Sicherheits-, Effizienz-, Zeit- und Komfortgewinne stellen sich nicht von selbst ein.

Voraussetzungen sind erstens eine geeignete Praxis bei der Zulassung von Fahrzeugen und Software, bei Haftungsfragen sowie bei der Ausstellung von Führerscheinen, Nutzer- oder Betreiberausweisen. Zweitens sind attraktive sowie akzeptierte Sharing- und Pooling-Angebote zur Individualisierung und Nachfrageadaption im kollektiven Verkehr notwendig. Die zunehmenden Mobilitätsansprüche können vor allem dann nachhaltig abgewickelt werden, wenn die Auslastung der Fahrzeuge erhöht wird. Drittens sind griffige Instrumente im Verkehrsmanagement zu finden, welche die verfügbaren Straßenkapazitäten optimal ausnutzen. Viertens muss die Vernetzung vorangetrieben werden, indem eine entsprechende Dateninfrastruktur aufgebaut wird.

Werden diese Voraussetzungen nicht rechtzeitig geschaffen, so resultiert eine klare Zunahme der Fahrleistungen insbesondere infolge von Leerfahrten, von Komfortsteigerung und Zeitgewinnen. Die Rahmenbedingungen müssen schrittweise und wirkungsvoll gesetzt werden. Dem politischen Gestaltungswillen und dem gesellschaftlichen Diskurs kommt also ein zentraler Stellenwert zu. Ohne angemessene Regelungen von Zustand zu Zustand sind Fehlentwicklungen wie zunehmende Staus und Verkehrsbehinderungen absehbar.

\section{Literatur}

Bösch, Patrick; Becker, Felix; Becker, Henrik; Axhausen, Kay (2017): Cost-based analysis of autonomous mobility services. Working Paper. ETH Zurich: Institute for Transport Planning and Systems (IVT).

Deutsche Bundesregierung (2015): Strategie automatisiertes und vernetztes Fahren. Leitanbieter bleiben, Leitmarkt werden, Regelbetrieb einleiten. Berlin: Bundesministerium für Verkehr und digitale Infrastruktur.

EBP - Ernst Basler + Partner (2017): Einsatz automatisierter Fahrzeuge im Alltag. Denkbare Anwendungen und Effekte in der Schweiz. Schlussbericht Grundlagenanalyse (Phase A). Zürich: EBP Schweiz AG.

Fellendorf, Martin (2017): Automatisierung im Mischverkehr. Verkehrswissenschaftliche Erkenntnisse, Überlegungen zur Leistungsfähigkeit. Technische Universität Graz: ÖVG-Forum Automatisierung im Verkehr.
Friedrich, Bernhard (2015): Verkehrliche Wirkung autonomer Fahrzeuge. In: Autonomes Fahren. Technische, rechtliche und gesellschaftliche Aspekte. Wiesbaden: Springer, S. 331-350, DOI: 10.1007/978-3-662-45854-9.

Hartmann, Martin; Motamedidehkordi, Nassim; Krause, Sabine; Hoffmann, Silja; Vortisch, Peter; Busch, Fritz (2017): Impact of automated vehicles on capacity of the German freeway network. Montreal: Conference Paper, ITS World Congress.

Meyer, Jonas; Becker, Henrik; Bösch, Patrick; Axhausen, Kay (2016): Impact of autonomous vehicles on the accessibility in Switzerland. ETH Zürich: Working Paper, Institute for Transport Planning and Systems (IVT).

OECD/ITF - Organisation für wirtschaftliche Zusammenarbeit und Entwicklung/ International Transport Forum (2015): Urban mobility system upgrade. How shared self-driving cars could change city traffic. Paris: International Transport Forum.

SAE International (2014): Automated driving, levels of driving automation are defined in New SAE International Standard J3016. Warrendale: Society of Automotive Engineers.

Schweizerischer Bundesrat (2016): Automatisiertes Fahren. Folgen und verkehrspolitische Auswirkungen. Bericht des Bundesrates in Erfüllung des Postulats Leutenegger Oberholzer 14.4169 „Auto-Mobilität", 0353-1246. Bern: Schweizer Eidgenossenschaft.

UITP - Internationaler Verband für öffentliches Verkehrswesen (2017): Policy brief, autonomous vehicles. A potential game changer for urban mobility. Brüssel: International Association of Public Transport.

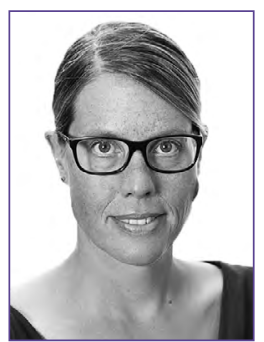

\section{FABIENNE PERRET}

ist Leiterin des Geschäftsbereichs Verkehr bei EBP Schweiz. Sie hat langjährige Erfahrung in der Planung und Beratung zu Gesamtverkehrs- und Mobilitätsfragen, sowohl für die öffentliche Hand als auch für private Unternehmen. Sie studierte Geomatikingenieurwissenschaften in Zürich und Victoria.

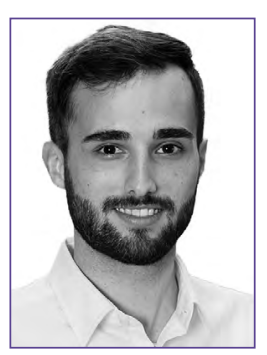

\section{REMO FISCHER}

arbeitet im Geschäftsbereich Verkehr bei EBP Schweiz mit den Schwerpunkten Verkehrsplanung und Verkehrstechnik. Als Bauingenieur erforscht er das Verhalten von Verkehrssystemen und wendet sein Fachwissen in der Planung und Projektierung an

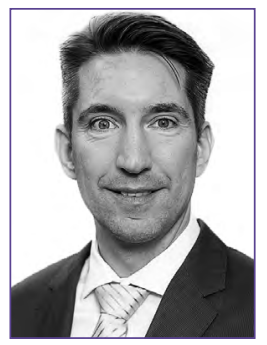

\section{HOLGER FRANTZ}

ist Geschäftsführer von EBP Deutschland. Er beschäftigt sich insbesondere mit Auswirkungsanalysen des Verkehrs, der Analyse von lufthygienischen und stadtklimatischen Situationen, mit Elektromobilität und weiteren zukünftigen Mobilitätsformen. 


\title{
Zur gesellschaftlichen Wahrnehmung des voll autonomen Fahrens und seiner möglichen nachhaltigkeits- bezogenen Implikationen
}

Ergebnisse einer Repräsentativbefragung

\begin{abstract}
Jürgen Hampel*, Universität Stuttgart, Institut für Sozialwissenschaften, Abteilung für Technik- und Umweltsoziologie, Seidenstraße 36, 70174 Stuttgart (Juergen.Hampel@sowi.uni-stuttgart.de)

Cordula Kropp, Universität Stuttgart, Institut für Sozialwissenschaften, Abteilung für Technik- und Umweltsoziologie, Seidenstraße 36, 70174 Stuttgart (cordula.kropp@sowi.uni-stuttgart.de)

Michael M.Zwick, Universität Stuttgart, Institut für Sozialwissenschaften, Abteilung für Technik- und Umweltsoziologie, Seidenstraße 36, 70174 Stuttgart (michael.zwick@sowi.uni-stuttgart.de)

* Die Autorlnnen sind in alphabetischer Folge aufgelistet.
\end{abstract}

Dieser Artikel thematisiert die Nachhaltigkeit des autonomen Fahrens. Die Daten des TechnikRadars, einer für die deutsche Wohnbevölkerung repräsentativen Bevölkerungsumfrage, aktuell zur Digitalisierung, erlauben es, methodisch belastbar Aussagen über die Wahrnehmung des autonomen Fahrens zu machen. Der Artikel fügt dem bislang vorrangig expertenbasierten Diskurs explizit die Stimme der Öffentlichkeit hinzu. Obwohl das System des autonomen Fahrens noch nicht hinreichend definiert ist, können für die weitere Entwicklung bedeutsame Aspekte aufgezeigt werden: Autonomes Fahren trifft derzeit nicht auf einen gesellschaftlichen Bedarf, die Delegation des Autofahrens an technische Systeme wird nur von einer Minderheit begrüßt. Skepsis finden wir vor allem bei der sozialen Nachhaltigkeit autonomer Mobilität; insbesondere werden Sicherheitsmängel und die Anfälligkeit des Systems, etwa gegenüber Angriffen von Cyber-Kriminellen, befürchtet.

\section{The social perception of fully autonomous driving and its possible sustainability-related implications \\ Results of a representative survey in Germany}

This article discusses the sustainability of autonomous driving. The empirical analyses presented in this article are based on data of the first

This is an article distributed under the terms of the Creative Commons Attribution License CCBY 4.0 (https://creativecommons.org/licenses/by/4.0/)

https://doi.org/10.14512/tatup.27.2.38

Submitted: 30.04 .2018 . Peer reviewed. Accepted: 07.06.2018
TechnikRadar, a survey on the perception of technology with representative for the German population. While most of the literature on autonomous driving focuses on expert views on this issue, this paper adds the perspective of the general public. Although autonomous driving as a socio-technical system is far from being fully developed, making it impossible to assess the ecological effects of its applications, the TechnikRadar allows studying the perceived social sustainability of autonomous driving. At the moment, public perception of autonomous driving is dominated by fears regarding safety, the protection of data produced by the system, and the system's vulnerability, in particular to attacks by cyber criminals.

Keywords: autonomous driving, sustainability, public perception

\section{Einleitung}

$\mathrm{Zu}$ den gegenwärtig intensiv diskutierten Themen, sowohl in der politischen Arena als auch in der Wissenschaft, gehört die Zukunft der Mobilität. Dazu haben eine Vielzahl von Entwicklungen beigetragen, die nicht zuletzt Folgen der massiven Verbreitung individueller Mobilität sind. Grunwald und Kopfmüller (2012, S. 117) nennen beispielhaft ,Treibhausgasemissionen, umwelt- und gesundheitsschädliche Schadstoffemissionen $\left(\mathrm{NO}_{\mathrm{x}}\right.$, 
Feinstaub usw.), Lärmbelastungen, Ressourcenverbrauch sowie Flächenversiegelung, Landschaftszerschneidung und hieraus resultierende Biodiversitätsverluste“.

Während das gegenwärtig dominante Mobilitätsmodell in das Zentrum gesellschaftlicher Auseinandersetzungen gerät, stehen neue technische Entwicklungen vor der Tür, von denen angenommen wird, dass sie Mobilität neu definieren. Zu diesen Neuerungen gehört das autonome Fahren, ein Konzept, bei dem Menschen nicht mehr als (alleinige) Fahrzeuglenker in Erscheinung treten, sondern die Kontrolle und Steuerung des Verkehrs durch (auch) vernetzte Computersysteme erfolgt. Noch steckt das autonome Fahren in den Kinderschuhen; gegenwärtig steht in erster Linie die technische Funktionsfähigkeit autonomer Fahrzeuge auf dem Prüfstand. Wie autonomes Fahren als Verkehrssystem organisiert werden wird, ist dagegen noch nicht absehbar, viele Möglichkeiten sind denkbar. Eine Abschätzung der Folgen autonomer Mobilität ist daher zum gegenwärtigen Zeitpunkt kaum sicher möglich. Gerade deshalb ist eine Diskussion über die Gestaltung des autonomen Verfahrens von erheblicher Bedeutung, als es darum geht, Parameter festzulegen, an denen sich das System autonomes Fahren orientieren soll.

Gegenwärtig wird dieser Diskurs vor allem als Expertendiskurs geführt. Das TechnikRadar, eine von ZIRIUS an der Universität Stuttgart durchgeführte und von acatech, der deutschen Akademie für Technikwissenschaften, und der Körber-Stiftung geförderte repräsentative Untersuchung der Einstellungen der deutschen Öffentlichkeit zum Thema Technik, die sich in ihrer 2018er Studie schwerpunktmäßig mit Fragen der Digitalisierung befasst hat, erlaubt es, diesen Expertendiskurs um die Perspektive der Öffentlichkeit zu ergänzen.

\section{Nachhaltige Mobilität}

Die Diskussion um die Zukunft der Mobilität findet dominant unter dem Label „Nachhaltige Mobilität“" statt (etwa Umweltbundesamt 2005), womit der allgemeine Nachhaltigkeitsdiskurs mit dem Mobilitätsdiskurs verbunden wird. Zahlreiche Projekte, von technischen Entwicklungsprojekten bis hin zu Reallaboren, die versuchen, alternative Mobilitätskonzepte zu initiieren, zielen darauf ab, Mobilität und das Mobilitätssystem in Richtung Nachhaltigkeit zu verändern.

So verbreitet Nachhaltigkeit als Prinzip ist, so schwer fällt es bis heute, Nachhaltigkeit präzise zu definieren. Auch wenn zahlreiche Versuche unternommen wurden, wissenschaftlich nachvollziehbare Nachhaltigkeitskonzepte zu entwickeln, so ist der Begriff Nachhaltigkeit nach wie vor alles andere als eindeutig bestimmt. Konkurrierende Vorstellungen darüber, was Nachhaltigkeit ist, sind auch in Zukunft zu erwarten, was nicht zuletzt daran liegt, dass Nachhaltigkeit weniger ein wissenschaftliches Prinzip als ein ethischer Imperativ ist (Grunwald und Kopfmüller 2012, S. 11). Die Brundlandt-Kommission hat Nachhaltigkeit als ein Prinzip formuliert, das die Bedürfnisse der gegenwärtigen Generation befriedigt, ohne die Möglichkeit künftiger
Generationen zu beeinträchtigen, ihre Bedürfnisse zu befriedigen (Hauff 1987). Dieses Nachhaltigkeitskonzept ist anthropozentrisch, es stellt den Menschen, genauer seine Bedürfnisse, in den Mittelpunkt. Die ökologische Frage stellt sich hier nur insofern, als eine nicht-nachhaltige Entwicklung die Möglichkeiten künftiger Generationen, ihre Bedürfnisse zu befriedigen, in Frage stellt. In der Rio-Erklärung von 1992 (Vereinte Nationen 1992) wurde die gesellschaftliche Dimension von Nachhaltigkeit noch einmal verstärkt, indem Prinzipien wie die Bekämpfung der Armut oder die Beteiligung aller betroffenen Bürgerinnen und Bürgern eingefordert wurden.

\section{Die Ergebnisse des TechnikRadars ergänzen den bisherigen Experten- diskurs zum autonomen Fahren um die Perspektive der Öffentlichkeit.}

In der Nachhaltigkeitsforschung sind Modelle verbreitet, die mehrere Nachhaltigkeitsdimensionen unterscheiden, am häufigsten zwischen ökologischer, sozialer und ökonomischer Nachhaltigkeit als Bewertungsdimensionen, zwischen denen durchaus konfliktäre Beziehungen möglich sind. Im HGF-Konzept (Kopfmüller et al. 2001) gehört die Bewahrung der Entwicklungs- und Handlungsmöglichkeiten genauso zur Nachhaltigkeit wie die Erhaltung des gesellschaftlichen Produktivpotenzials und die Sicherung der menschlichen Existenz.

Renn et al. 2007 gehen von drei normativ gesetzten Nachhaltigkeitsfunktionen aus (2007, S. 41), nämlich der Sicherstellung gesellschaftlicher, ökonomischer und ökologischer Funktionen für kommende Generationen (systemare Integrität), der Durchsetzung von Gerechtigkeitsnormen innerhalb und zwischen Generationen und dem dauerhaften Erhalt der individuellen Lebensqualität, wenn auch die Unterscheidung bei der Festlegung der Indikatoren nicht aufrechterhalten wird. So wird der Anteil des öffentlichen Personenverkehrs an der Personenbeförderung als Indikator für Lebensqualität definiert (2007, S. 77).

Grunwald und Kopfmüller (2012, S. 117) sehen Mobilität als wesentliches gesellschaftliches Bedürfnis. Mobilität sei einerseits Voraussetzung für Teilhabe, andererseits Voraussetzung für Beschäftigung und Wohlstand. Sie definieren Nachhaltigkeit im Verkehrsbereich, sich auf andere Studien beziehend, als „Zugang möglichst aller Menschen zu sicheren Verkehrsdienstleistungen, die ihnen die Teilhabe am gesellschaftlichen Leben ermöglichen, die erschwinglich sind und die möglichst geringe Umwelt- und Gesundheitsbelastungen bewirken" (Grunwald und Kopfmüller 2012, S. 119 f.). Diese Definition, die wir für diese Arbeit übernehmen, unterscheidet die folgenden Bewertungsdimensionen für nachhaltige Mobilität: Umwelt- und Gesundheitsbelastungen, Teilhabemöglichkeiten, Kosten und Sicherheit. 
In Anbetracht des gegenwärtigen Entwicklungsstands autonomer Mobilität ist es noch nicht möglich, hinreichend präzise die Nachhaltigkeit dieser Innovation und vor allem der mit ihr verbundenen Nutzungen und Anwendungen zu analysieren. Die für die Auswirkungen entscheidendere Frage, wie autonomes Fahren als sozio-technisches System konfiguriert wird, ist noch nicht einmal in Ansätzen geklärt (vgl. auch Schreurs und Steuwer 2015, S. 161; Dangschat 2017, S. 496, Thomopoulos und Givoni 2015, S.9). Wird sich eine bloße technische Aufrüstung des jetzigen Individualverkehrs durchsetzen? Werden sich Callby-demand-Systeme durchsetzen, bei denen sich Nutzer ein Gefährt wie ein Taxi für einen bestimmten Fahrtweg mieten kön- den können, wäre durch autonome Mobilität eher noch eine $\mathrm{Zu}$ nahme des Individualverkehrs (Thomopoulos und Givoni 2015, S. 9) und ein Beitrag zu einer weiteren Zersiedelung der Landschaft (Dangschat 2017, S. 504) zu erwarten.

Auch wenn hinsichtlich der Gestaltung von autonomem Fahren noch zahlreiche Entscheidungen ausstehen, ist es an der Zeit, dem bislang eher von Experten getragenen Diskurs - seien es Technikexperten, seien es Experten aus dem Gebiet der Technikfolgenabschätzung - die Perspektive der Öffentlichkeit gegenüberzustellen. Wir wollen in unserem Beitrag einer bedürfnisorientierten Nachhaltigkeitskonzeption, wie sie von der Brundlandt-Kommission vorgeschlagen wird, und der Definition

\section{Es ist an der Zeit, dem bislang eher von Experten getragenen Diskurs über autonomes Fahren die Perspektive der Öffentlichkeit gegenüberzustellen.}

nen (ride sharing)? Oder sind andere Formen zu erwarten, etwa in der Art von Sammeltaxen, die nachfragebedingt optimierte Routen bedienen? Oder wird es lediglich eine gleichmäßige Geschwindigkeitssteuerung aller Fahrzeuge geben, um die Kapazität des Straßensystems optimieren zu können? Je nach Gestaltung des autonomen Fahrens als System werden die Auswirkungen in ökologischer, ökonomischer und sozialer Hinsicht unterschiedlich aussehen.

Vor diesem Hintergrund ist es nicht überraschend, dass auch in der Literatur die Auswirkungen des autonomen Fahrens als ungewiss eingeschätzt werden (Wadud et al. 2016, S. 16). Autonomes Fahren wird als Thema der industriellen Wettbewerbsfähigkeit, der Ressourcen-Effizienz und der Nachhaltigkeit gesehen (Schreurs und Steuwer 2015, S. 155). Dabei kommen die meisten Studien zu optimistischen Ergebnissen, was nicht zuletzt daran liegt, dass sie sich mit Fragen der technischen Machbarkeit beschäftigen. Die Literatur zum autonomen Fahren diskutiert die Auswirkungen dieser Innovation daher vorrangig auf der Grundlage von Erwartungen und Visionen, ist also eher Vision Assessment als Technology Assessment im engeren Sinn. Dabei gibt es nicht nur positive, sondern auch negative Utopien des autonomen Fahrens.

Positive Erwartungen beziehen sich auf die Effizienzgewinne bei der Mobilität (u. a. Funk 2015, S. 867; Friedrich 2015, S. 312), aber auch auf die Aussicht, die Teilhabemöglichkeiten von mobilitätseingeschränkten Menschen zu erhöhen (Grunwald 2015, S. 668; Dangschat 2017, S. 497). Zu den befürchteten negativen Auswirkungen zählt die Zunahme der Mobilität gerade dadurch, dass Mobilität bequemer wird und autonome Mobilität auch in einem Konkurrenzverhältnis zu öffentlichen Verkehrsmitteln und zu nicht-motorisierten Formen individuellen Personennahverkehrs steht (Dangschat 2017; S. 497 f.). Bedenkt man zudem, dass Pendelzeiten für andere Tätigkeiten genutzt wer- nachhaltiger Mobilität von Grunwald und Kopfmüller (2012) folgen, und uns der Frage zuwenden, ob und wie autonomes Fahren nach Einschätzung der Öffentlichkeit zu einer nachhaltigen Mobilität beitragen kann.

Da die ökologischen Auswirkungen des voll autonomen Fahrens noch nicht abgeschätzt werden können, steht in diesem Beitrag die soziale Dimension der Nachhaltigkeit im Mittelpunkt, insbesondere die Frage nach der Ermöglichung von Teilhabe ansonsten ausgeschlossener Menschen und die Frage nach der Sicherheit der Mobilität, wozu die Sicherheit vor Unfällen gehört, aber auch, auf einer abstrakteren Ebene, die Sicherheit des Verkehrssystems autonomer Mobilitität.

\section{Der Datensatz}

Die Frage, wie die deutsche Öffentlichkeit zum voll autonomen Fahren steht, welche Erwartungen und Bedenken sie an diese Technologie heranträgt, unter welchen Bedingungen ihre Nutzung in Frage käme, werden wir anhand der aktuellen TechnikRadar-Befragung diskutieren. Beim TechnikRadar handelt es sich um eine turnusmäßige Repräsentativbefragung der über 16-jährigen, deutschsprachigen Wohnbevölkerung, die Haltungen der Öffentlichkeit gegenüber zukunftsweisenden Technologien zum Gegenstand hat. Die Studie reagiert - sieht man von einigen wenigen Eurobarometer-Befragungen $\mathrm{ab}$ - auf den seit der Jahrtausendwende anhaltenden Mangel an empirischer Technikberichterstattung (Hampel und Zwick 2016, S. 28).

Die erste Welle des Technik-Radars ist dem Thema Digitalisierung und Automatisierung gewidmet, mit den Schwerpunkten Smart Home, Pflegeroboter und Autonomes Fahren. Für autonomes Fahren werden in der Literatur vier Entwicklungsstadien diskutiert, die sich vor allem in Bezug auf die Kontrolle durch 


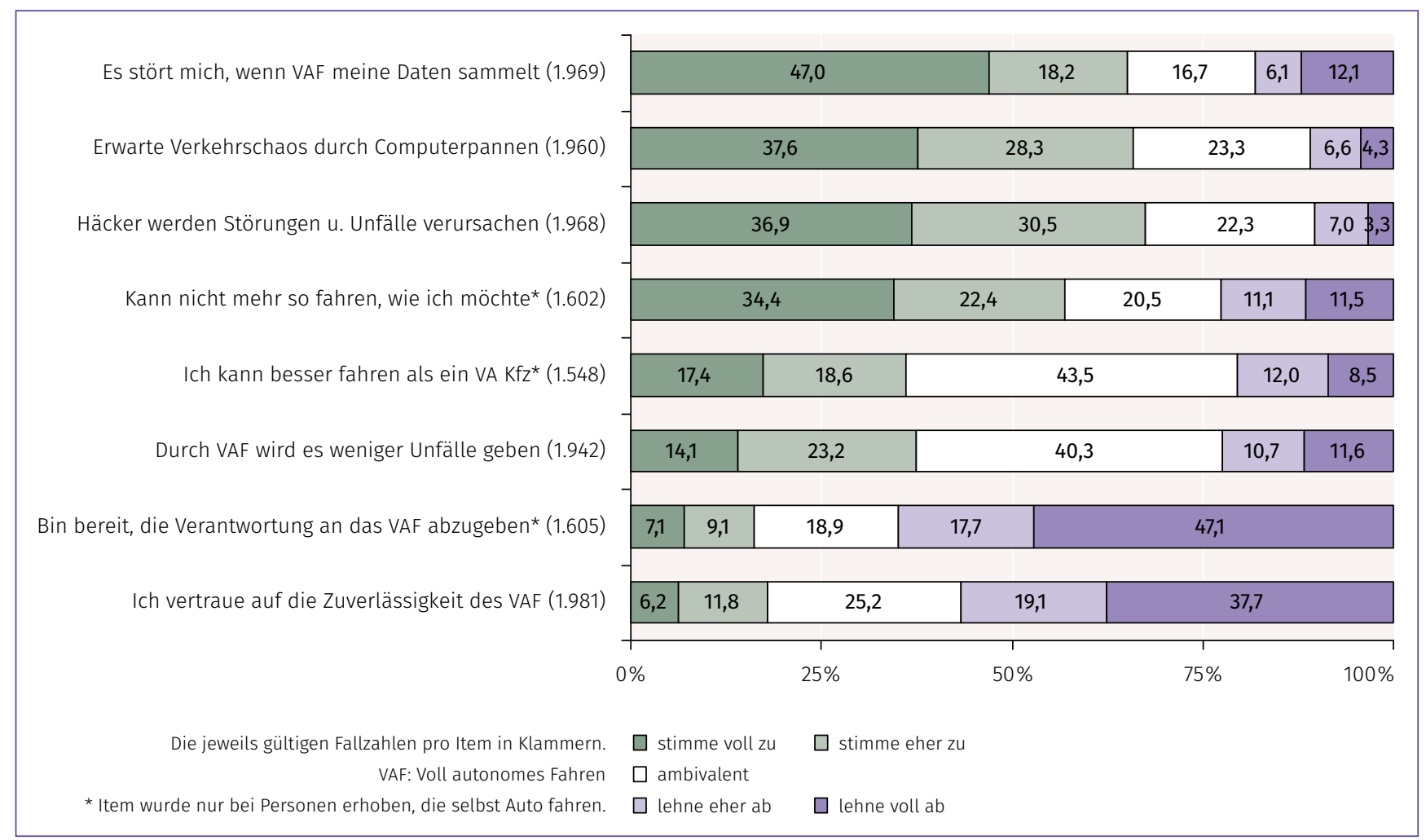

Abb.1: Die Einstellung der Öffentlichkeit zum vollautonomen Fahren.

Quelle: Eigene Darstellung auf Basis der Technik-Radar-Befragung 2017

Fahrzeuglenker unterscheiden": Stufe 1 bezeichnet das assistierte Fahren, etwa durch Antiblockiersysteme oder Spurhalteassistenten. Stufe 2 meint Teilautomatisierung, worunter beispielsweise Einparkhilfen oder so genannte Stauassistenten fallen. Entwicklungen dieser beiden Stufen werden bereits eingesetzt. Stufe 3 steht für hoch automatisiertes Fahren, worunter das selbständige Fahren auf der Autobahn oder im Stau fällt und die Umweltbeobachtung von den Fahrzeugtechnologien vollständig übernommen wird. Das vollautomatische Fahren im Stadtverkehr oder die fahrerlose Parkplatzsuche gipfeln in Stufe 4. Sie werden als vollautomatisiertes Fahren klassifiziert (Verband der Automobilindustrie 2015, S. 15), das nach den Ergebnissen einer Studie des Fraunhofer-Instituts für Arbeitswirtschaft und Organisation (IAO) in Deutschland vermutlich nicht vor 2030 die Marktreife erreichen wird (Cacilo et al. 2015, S. 9). Die thematische Breite und die begrenzte Befragungsdauer erzwangen allerdings Selektionen. Mit Blick auf die gewünschte zukunftsweisende Perspektive der Befragung fand deshalb ausschließlich das vollautonome Fahren Eingang in das TechnikRadar.

Die telefonische Befragung (CATI) wurde im Oktober und November 2017 von der INFO GmbH Markt- und Meinungs-

1 Die im TechnikRadar verwendete Klassifizierung von Stufen des autonomen Fahrens unterscheidet sich von anderen Klassifizierungssystemen, auf die in diesem TATuP-Thema verwiesen wird. Siehe dazu die Einleitung von Torsten Fleischer und Jens Schippl. forschung auf Basis einer Zufallsstichprobe im Dual-frameModus durchgeführt. Die Stichprobengröße beträgt 2.002 Fälle. Die Ausschöpfungsquote lag bei $15 \%$, ein Wert, den Gabler und andere nach einer Analyse von Telefonbefragungen als durchschnittlich bezeichnen (2012, S. 165): gegenwärtig seien Ausschöpfungsquoten zwischen $10 \%$ und $20 \%$ normal.

\section{Das voll autonome Fahren im Urteil der deutschen Öffentlichkeit}

Natürlich wäre es vermessen anzunehmen, mit einer Stichprobe aus dem Jahr 2017 eine Prognose über die gesellschaftliche Akzeptanz des voll autonomen Fahrens in ein oder zwei Dekaden abgeben zu können. Zu groß sind die Unwägbarkeiten über Art, Leistungsfähigkeit, Zuverlässigkeit und Kosten der Technik. Wie sich autonomes Fahren entwickeln wird, hängt wesentlich von der staatlichen Regulierung beispielsweise hinsichtlich Datenschutz, Datensicherheit oder Haftungsfragen ab, sowie von der Frage, über welche Geschäftsmodelle und für welche Klientel autonom steuernde Fahrzeuge vorrangig eingeführt werden für Personenverkehr, in der Logistik, über Sharing- bzw. Mobility-on-demand-Konzepte oder als Kraftfahrzeuge in Privatbesitz? Von der Beantwortung dieser Fragen hängen nicht nur der Markterfolg und die politische Akzeptanz des voll autonomen Fahrens maßgeblich ab, sondern auch die denkbaren Implika- 
tionen für die soziale, ökonomische und ökologische Nachhaltigkeit dieser Technik.

Die Versprechen der Produzenten klingen vollmundig: Neben der möglichen Abnahme der Zahl an Fahrzeugen durch voll autonome Fahrzeuge in Verbindung mit Sharing-Konzepten und der Lösung der innerstädtischen Parkplatzprobleme mittels Sammelgaragen und Valet-Parken versprechen die Produzenten auch, dass Staus durch eine erhöhte Verkehrsdichte mit gleichmäßigerer Geschwindigkeit vermieden werden können, dass vernetzte Fahrzeuge in engerem Abstand fahren und höhere Kapazitäten von Verkehrswegen ermöglichen. Vor allem aber mit zwei Hauptargumenten wird geworben: mit verbesserter Mobilität für Personen, die aus welchen Gründen auch immer, keine Fahrerlaubnis besitzen und mit sinkenden Unfallzahlen (exemplarisch Minx und Dietrich 2015; Heymann und Meister 2017, S. 7 f.; Fraedrich et al. 2017, S. 20 ff.).

Wie aber denkt die Öffentlichkeit hierzulande über das voll autonome Fahren? Abbildung1 zeigt, dass die Bevölkerung den Versprechungen der Hersteller zum gegenwärtigen Zeitpunkt wenig Glauben schenkt. Schon vor den beiden jüngsten, tödlich verlaufenen Unfällen mit voll autonom fahrenden Kraftfahrzeugen $^{2}$ äußerten gerade einmal 16,2\% der Befragten die Bereitschaft, ihre Verantwortung an voll autonom fahrende Fahrzeuge abzugeben. Es darf vermutet werden, dass dieses Maß der Akzeptanz nach den Ereignissen im März 2018 in der Öffentlichkeit noch weiter gesunken ist. Für diese These spricht auch, dass im TechnikRadar nur 18,0\% der Befragten auf die Zuverlässigkeit voll autonom fahrender Kraftfahrzeuge vertrauen und nur eine Minderheit von 37,3\% daran glauben mochte, dass es durch voll autonome Fahrzeuge weniger Unfälle geben wird. ${ }^{3}$

Das für die soziale Nachhaltigkeit erste relevante Kriterium, nämlich die in Aussicht gestellte Absenkung der Unfallzahlen, findet in der Öffentlichkeit nur geringe Glaubwürdigkeit. Statt-

2 Vor der Befragung lag nur der fatale Unfall eines selbstfahrenden Tesla S in Florida am 7. Mai 2016 (Spiegel Online 2016), wohingegen sich die beiden jüngsten tödlichen Unfälle mit selbstfahrenden Autos am 19. März 2018 in Arizona (Spiegel Online 2018 a) und am 23. März 2018 in Kalifornien nach der Erhebung des TechnikRadars ereigneten (Spiegel Online 2018 b).

3 Diese Zahlen liegen in der Größenordnung anderer aktueller Studien zum autonomen Fahren, die jedoch auf anderen Stichprobendesigns und unterschiedlichen Itemformulierungen basieren: In der für Autofahrer in Deutschland repräsentativen ARAL-Studie vom März 2017 konnten sich $20 \%$ der Befragten vorstellen, autonom zu fahren (in der Befragungswelle von 2015 waren es noch $34 \%$ gewesen); unter den Befragten mit kritischer Haltung dominierten Sicherheitsbedenken und eine hohe Unfallgefahr (ARAL AG 2017, S. 22). Der Deloitte Konsumenten-Studie zufolge präferierten 2016 in Deutschland rund $25 \%$ der Befragten voll autonomes Fahren; $72 \%$ der Befragten zweifeln an der Sicherheit der Fahrzeuge (Deloitte 2017, S. 4 ff.). In einer Repräsentativbefragung der erwachsenen Wohnbevölkerung in Deutschland zum autonomen Fahren im Auftrag von Ernst \& Young bekundeten 2017 26\% der Befragten, sich vorstellen zu können, zukünftig mit einem autonom fahrenden Fahrzeug unterwegs zu sein; $67 \%$ sahen in der mangelnden Sicherheit dieser Fahrzeuge ein grundsätzliches Problem - in der Befragungswelle 2013 waren es nur 44\% gewesen (Fuß 2017, S. 4 und S. 8). Auch in einer für die über 14-jährige Wohnbevölkerung repräsentativen Studie der Bertelsmann-Stiftung aus dem Jahr 2017 dominierten Angst vor Unfällen bzw. fehlerhafte Technik die Einwände gegen das autonome Fahren (Bertelsmann Stiftung 2017, S. 4). dessen werden weitere Einwände gegen das voll autonome Fahren vorgebracht, die auf eine Bedrohung von Freiheits- oder gar Grundrechten hinauslaufen, wie beispielsweise das Ende der von einem Premiumhersteller intensiv beworbenen „Freude am Fahren“" ${ }^{\prime 4}$ das von $56,8 \%$ der Befragten befürchtet wird, oder aber knapp zwei Drittel der Befragten (65,2\%), die sich daran stören, wenn voll autonom fahrende Fahrzeuge personenbezogene Daten sammeln. Ähnlich häufig werden Bedenken hinsichtlich der Systemsicherheit laut. Jeweils über $65 \%$ der Befragten erwarten, dass es zu Unfällen, Störungen oder gar einem Verkehrschaos kommen wird, wenn Computerpannen auftreten oder das System von Internetkriminellen gehackt wird. Lediglich bei der Frage, ob man selber besser fahren könne als der Computer, zeichnet sich ein uneinheitliches, ambivalentes Bild ab.

Insgesamt weisen die ausgeprägten Bedenken in Richtung einer als unzureichend wahrgenommenen Sicherheit und Systemzuverlässigkeit und machen die Gefahr datenschutzrechtlicher Übergriffe durch Produzenten und Betreiber zum gegenwärtigen Zeitpunkt für die geringe Akzeptanz dieser Technik verantwortlich - Aspekte, die einem Markterfolg im Privatkundensegment abträglich sind. Aus der Kritik der Befragten lässt sich institutionelle Verantwortung ableiten (Grunwald 2015, S. 682 ff.): Die Produzenten haben nicht nur für sichere, zuverlässige und resiliente Technik zu sorgen, sondern auch die verfassungsrechtlich garantierte informationelle Selbstbestimmung ihrer Kunden durch Einhaltung des Datenschutzes zu gewährleisten, die Politik hat die Sicherheit der Systeme und die Einhaltung des Datenschutzes zu überprüfen - auf diese Schlussfolgerungen weist auch eine aktuelle Studie des TÜV Rheinland hin (TÜV Rheinland 2018). Unsere Befunde geben Anlass zu der Vermutung, dass zumindest das Privatkundengeschäft in erheblichem Maße von der Glaubwürdigkeit und Leistungsfähigkeit der beiden Institutionen bei Datenschutz und Qualitätssicherung abhängt. Kritische Medienkommentare zu den jüngsten Unfällen mit voll autonomen Fahrzeugen mahnen, dass Feldversuche mit unausgereifter Technik dem Image autonomer Fahrzeuge und ihrer Produzenten nachhaltig schaden könnten (exemplarisch Pretzlaff 2016; Spiegel Online 2018 c; Doll 2018; Grunwald 2015, S. 680 ff.). ${ }^{5}$ Grunwald schreibt hierzu: „Wenn ein autonomes Fahrzeug wegen eines Softwarefehlers einen Unfall verursacht, ist das nicht akzeptabel. In einem solchen Fall ist anders als bei ,normalen ' Unfällen - eine erhebliche massenmediale Aufmerksamkeit zu erwarten. Insbesondere durch die

4 Exemplarisch sei an das Motto „Freude am Fahren“ von BMW (2018) erinnert, das der Automobilhersteller explizit mit „dynamischer Sportlichkeit“ verbindet. 5 Vorangegangene Unfälle, die von voll autonom fahrenden Fahrzeugen ausgingen, wurden schon Ende März 2018 von Tagesschau.de mit der Headline „Autonomes Fahren: Die Angst vor dem Autopiloten“ kommentiert (Tagesschau. de 2018 a). Am 24. Mai 2018 berichtete die Tagesschau über den Untersuchungsbericht zum Uber-Unfall in Arizona, am 30. Mai 2018 über die Kollision eines voll autonom fahrenden Tesla mit einem Polizeifahrzeug in Kalifornien (Tagesschau 2018 b und 2018 c). Eine Reihe von Kommentaren auf die Meldungen und das darin berichtete Technikversagen lassen erahnen, mit welch negativen Wirkungen durch derartige Unfälle auf die Technikakzeptanz gerechnet werden muss (Tagesschau.de $2018 \mathrm{~d}$ und $2018 \mathrm{e}$ ). 
Macht der Bilder und deren massenmediale Verbreitung können derartige Vorfälle eine erhebliche Wirkung [...] haben." (Grunwald 2015, S. 670)

Betrachten wir das zweite nachhaltigkeitsrelevante Kriterium: den in Aussicht gestellten verbesserten Zugang zu Mobilität und sozialer Teilhabe für Personen ohne Fahrerlaubnis, etwa durch Mobility-on-Demand-Angebote. Doch wie sehen das die Betroffenen selbst? In unserem Sample, das für die über 16-jährige deutschsprachige Wohnbevölkerung Repräsentativität beansprucht, haben wir untersucht, ob Personen, die zum Befragungszeitpunkt selbst keine Fahrerlaubnis hatten, Personen, die über 80 Jahre alt waren oder ihren Gesundheitszustand als eher schlecht oder schlecht einschätzen, durch eine überdurchschnittlich positive Haltung gegenüber dem voll autonomen Fahren auffallen. Keine der in Abbildung 1 aufgeführten Eigenschaften des autonomen Fahrens ist in nennenswerter Weise mit dem Besitz einer Fahrerlaubnis, hohem Alter oder schlechtem Gesundheitszustand assoziiert. ${ }^{6}$ Offenbar wird von diesem prädisponierten Personenkreis das voll autonome Fahren nicht in besonderer Weise als willkommenes Mittel zur Verbesserung von Mobilität oder sozialer Teilhabe wahrgenommen, ein Argument, das sich nicht zugunsten der sozialen Nachhaltigkeit dieser Technik verwenden lässt.

Die ökologische Dimension der Nachhaltigkeit kann erst beurteilt werden, wenn die Technologie eingeführt ist. Die von Fraedrich et al. (2017, S. 21 ff.) angestellten Überlegungen verdeutlichen, dass das vollautonome Fahren aus ökologischer Perspektive zu gegenläufigen Wirkungen führen kann, deren Eintreten von verbrauchsarmen Fahrzeugen ein Guthaben für nicht-nachhaltige Fahrten gesammelt zu haben. Dennoch möchten wir die referierten Daten durch den Befund ergänzen, dass 57,0\% aller Befragten angeben, bei adäquatem Angebot, umweltfreundliche Verkehrsmittel bei der Verkehrsplanung zu bevorzugen, nur $22,8 \%$ lehnen das ab. Knapp zwei Drittel der von uns Befragten $(63,1 \%)$ geben an, im Alltag auf die Nutzung eines Pkw angewiesen zu sein. Von diesen äußern 47,4\% die verbale Absicht, bei einem guten ÖPNV-Angebot auf das Auto verzichten zu wollen. Es besteht keinerlei Zusammenhang zwischen dieser Einstellung und der Haltung zum autonomen Fahren.

\section{Resümee}

Will man Indizien diskutieren, die für oder gegen die Nachhaltigkeit des voll autonomen Fahrens sprechen, gilt es, zuvor einen kritischen Blick auf das Nachhaltigkeitskonzept zu werfen. Gestützt auf das Nachhaltigkeitsverständnis der Brundtland-Kommission (Hauff 1987) und die Definition nachhaltiger Mobilität von Grunwald und Kopfmüller (2012) plädieren die Autoren für ein dreidimensionales Nachhaltigkeitskonzept, in dem soziale, ökonomische und ökologische Nachhaltigkeit gleichwertig koexistieren. Die Frage nach der Nachhaltigkeit des voll autonomen Fahrens ist im Hinblick auf diese drei Dimensionen zu diskutieren. Mit der Marktreife des voll autonomen Fahrens wird hierzulande frühestens in einem Jahrzehnt gerechnet, weswegen es gegenwärtig nicht möglich ist, anhand von Umfragedaten be-

\section{Sicherheit und Systemzuverlässigkeit des autonomen Fahrens werden als unzureichend wahrgenommen. Dies erklärt die gegenwärtig geringe Akzeptanz der Technik.}

und Wirkmächtigkeit vorab nicht eingeschätzt werden können. Allzu oft erweisen sich selbst solche Innovationen, die explizit mit dem Versprechen einer Verringerung ökologischer Belastungen entwickelt und eingeführt wurden, im Rückblick aufgrund der bekannten Rebound-Effekte (exemplarisch Sonnberger und Deuschle 2014) als nicht nachhaltig, sondern verursachen additive Nutzungen oder ein moral licensing - also die individuelle Einschätzung, auf dem moralischen Konto durch die Nutzung

\footnotetext{
6 Bei den vorliegenden Daten wird die Stärke des statistischen Zusammenhangs jeweils mit dem Kontingenzkoeffizienten Cramers V ausgedrückt, der Werte zwischen 0 (,kein Zusammenhang ${ }^{\prime}$ ) und 1 (,perfekter Zusammenhang ${ }^{\prime}$ ) annehmen kann. In der Praxis gelten erst Werte ab 0,1 als substanziell und interpretationsfähig (Cleff 2015, S. 85). Bei allen der hier untersuchten rund zwei Dutzend Zusammenhängen blieb $V \leq 0,08$. Auch in einer einschlägigen Studie von Bertelsmann nimmt die Begeisterung für das autonome Fahren entlang der Altersgruppen streng monoton ab und das Misstrauen mit wachsendem Alter zu (Berteilsmann Stiftung 2017, S. 4).
}

lastbare Prognosen über soziale, ökonomische oder ökologische Nachhaltigkeitspotenziale abzugeben. Gleichwohl haben wir in unserem Beitrag einige Indizien zusammengetragen, die für die Einschätzung von Nachhaltigkeitspotenzialen relevant sind.

Für die soziale Nachhaltigkeit sprechen vor allem steigende Mobilitäts- und Teilhabechancen von Personen ohne Fahrerlaubnis, allen voran alte, kranke oder behinderte Menschen - Personengruppen, die sich in unserem Survey allerdings nicht durch ein überdurchschnittliches Interesse am voll autonomen Fahren auszeichnen. Möglicherweise wird ihre Zustimmung von den noch unbekannten Geschäftsmodellen, Preisen, Bedienbarkeit und gegebenenfalls der Kostenübernahmebereitschaft durch den Sozialstaat abhängen.

Die ökonomische Nachhaltigkeit wird sich am Markterfolg des voll autonomen Fahrens bemessen, und zwar in allererster Linie am Massenmarkt mit Privatkunden. Die Daten des TechnikRadars zeigen allerdings, dass es aufgrund einiger Ein- 
wände um die Akzeptabilität des voll autonomen Verfahrens in der deutschen Öffentlichkeit gegenwärtig nicht gut bestellt ist. Die Sicherheit, ein Bestandteil der Definition nachhaltiger Mobilität von Grunwald und Kopfmüller (2012), wird von den Befragten des TechnikRadars sehr kritisch eingeschätzt. Defizite werden vor allem in nicht erfüllten institutionellen Verantwortlichkeiten gesehen, wie etwa in der technischen Zuverlässigkeit, Verkehrssicherheit, Datenschutz und Datensicherheit. Darüber hinaus werden die Geschäftsmodelle und Preise erheblich über den Markterfolg entscheiden und nicht zuletzt, in wieweit es gelingt, die „Freude am Fahren“ glaubhaft mit neuen Inhalten $\mathrm{zu}$ füllen.

\section{Entscheidend für den Markterfolg \\ des autonomen Fahrens werden}

\section{Geschäftsmodelle, Preise und neue Inhalte für ,Freude am Fahren“.}

Die ökologischen Folgen des voll autonomen Fahrens sind unseres Erachtens am schwierigsten zu beurteilen, hängen sie doch nicht nur maßgeblich vom Markterfolg ab, sondern von einer Reihe derzeit ungewisser Fragen zur technischen Beschaffenheit (Antriebsform, Motorleistung) und zur planerischen und sozialen Einbettung (z. B. Privatbesitz vs. Sharing- bzw. Mobility-on-demand-Konzepte; Nutzung übergeordneter Steuerungsmöglichkeiten; Auswirkungen auf die ÖPNV-Nutzung). Es darf aber vermutet werden, dass mit steigenden Beteiligungschancen bisher vom motorisierten Individualverkehr ausgeschlossener Personen und einem großen Markterfolg des voll autonomen Fahrens das Verkehrsaufkommen insgesamt ansteigen und damit die sozialen und ökonomischen Dimensionen von Nachhaltigkeit in ein Spannungsverhältnis zu den Postulaten einer ökologischen Nachhaltigkeit geraten werden.

\section{Literatur}

Aral AG (Hg.) (2017): Aral Studie. Trends beim Autokauf 2017. Bochum: Aral Aktiengesellschaft. Online verfügbar unter www.aral.de/content/dam/aral/ Presse\%20Assets/pdfs-Broschueren/Aral-Studie-Trends-beim-Autokauf-2017. pdf, zuletzt geprüft am 11.06.2018.

Bertelsmann Stiftung (Hg.) (2017): Automatisiertes Fahren. Aktuelle Einstellungen in Deutschland. Gütersloh. Online verfügbar unter https://www.bertelsmannstiftung.de/fileadmin/files/BSt/Publikationen/GrauePublikationen/ Factsheet_LK_Automatisiertes-Fahren_Aktuelle-Einstellungen-inDeutschland_2017.pdf, zuletzt geprüft am 11.06.2018.

BMW (2018): Freude am Fahren hat einen Namen. Online verfügbar unter www.bmwgroup.com/de/marken/bmw.html, zuletzt geprüft am 11.06.2018.

Cacilo, Andrej et al. (2015): Hochautomatisiertes Fahren auf Autobahnen. Industriepolitische Schlussfolgerungen. Stuttgart: Fraunhofer Institut für Arbeitswirtschaft und Organisation.
Cleff, Thomas (2015): Deskriptive Statistik und moderne Datenanalyse. Eine computergestützte Einführung mit Excel, SPSS und STATA. Wiesbaden: Springer.

Dangschat, Jens (2017): Automatisierter Verkehr - was kommt auf uns zu? In: Zeitschrift für Politikwissenschaft 27 (4), S. 493-507.

Deloitte (Hg.) (2017): What's ahead for fully autonomous driving? Consumer opinions on advanced vehicle technology. Perspectives from Deloitte's global automotive consumer study. Online verfügbar unter www2.deloitte.com/ content/dam/Deloitte/de/Documents/manufacturing/us-manufacturingconsumer-opinions-on-advanced-vehicle-technology.pdf, zuletzt geprüft am 16.05.2018.

Deutsche Bank Research (Hg.) (2017): Das „digitale Auto“. Mehr Umsatz, mehr Konkurrenz, mehr Kooperation. Deutschland-Monitor Digitale Ökonomie und struktureller Wandel. Frankfurt a. M. Online verfügbar unter www.dbresearch. de/PROD/RPS_DE-PROD/PROD00000000000445411/Das_\%E2\%80\%9Edigitale_ Auto\%E2\%80\%9C\%3A_Mehr_Umsatz\%2C_mehr_Konkurrenz\%2C.pdf, zuletzt geprüft am 11.06.2018.

Die Welt (2018): Lizenz weg. Unfall mit Roboter-Auto hat Folgen für Uber. Online verfügbar unter www.welt.de/wirtschaft/article174937737/Autonomes-FahrenToedlicher-Uber-Crash-wird-Roboterautos-nicht-bremsen.html, zuletzt geprüft am 11.06.2018.

Ernst \& Young GmbH (Hg.) (2017): Autonomes Fahren in Deutschland. Ergebnisse einer Befragung von 1.000 Verbrauchern. Eschborn: Ernst \& Young GmbH. Online verfügbar unter www.ey.com/Publication/vwLUAssets/ey-autonomesfahren-in-deutschland/\$FILE/ey-autonomes-fahren-in-deutschland.pdf, zuletzt geprüft am 11.06.2018.

Fraedrich, Eva et al. (2017): Automatisiertes Fahren im Personen- und Güterverkehr. Auswirkungen auf den Modal-Split, das Verkehrssystem und die Siedlungsstrukturen. In: e-mobil BW GmbH - Landesagentur für Elektromobilität und Brennstoffzellentechnologie Baden-Württemberg ( $\mathrm{Hg}$.): Publikationen der e-mobil BW. Stuttgart. Online verfügbar unter www.e-mobilbw.de/files/e-mobil/content/DE/Publikationen/PDF/PDF_2017/ Studie_AutomatisiertesFahren.pdf, zuletzt geprüft am 11.06.2018.

Friedrich, Bernhard (2015): Verkehr. In: Markus Maurer, Christian Gerdes, Barbara Lenz und Hermann Winner (Hg.): Autonomes Fahren. Technische, rechtliche und gesellschaftliche Aspekte. Heidelberg: Springer, S. 309-311.

Funk, Jeffrey (2015): IT and sustainability. New strategies for reducing emissions and resource usage in transportation. In: Telecommunications Policy 39 (10), S. 861-874.

Gabler, Siegfried; Häder, Sabine; Lehnhoff, Iris; Maridian, Elisabeth (2012): Weighting for unequal inclusion probabilities and nonresponse in dual frame telephone surveys. In: Sabine Häder, Michael Häder und Mike Kühne (Hg.): Telephone surveys in Europe. Research and practice. Heidelberg: Springer, S. 147-167.

Grunwald, Armin (2015): Gesellschaftliche Risikokonstellationen für autonomes Fahren. Analyse, Einordnung und Bewertung. In: Markus Maurer, Christian Gerdes, Barbara Lenz und Hermann Winner (Hg.): Autonomes Fahren. Technische, rechtliche und gesellschaftliche Aspekte. Heidelberg: Springer, S. 661-685.

Grunwald, Armin; Kopfmüller, Jürgen (2012): Nachhaltigkeit. Frankfurt a. M.: Campus.

Hampel, Jürgen; Zwick, Michael (2016): Wahrnehmung, Bewertung und Akzeptabilität von Technik. In: TATuP - Zeitschrift für Technikfolgenabschätzung in Theorie und Praxis, 25 (1), S. 24-38. 
Hauff, Volker (Hg.) (1987): Unsere gemeinsame Zukunft. Der Brundtland-Bericht der Weltkommission für Umwelt und Entwicklung. Greven: Eggenkamp.

Kopfmüller, Jürgen et al. (2001): Nachhaltige Entwicklung integrativ betrachtet. Konstitutive Elemente, Regeln, Indikatoren. Berlin: edition sigma.

Minx, Eckard; Dietrich, Rainer (2015): Autonomes Fahren. Wo wir heute stehen und was noch zu tun ist. Ladenburg: Piper.

Renn, Ortwin; Deuschle, Jürgen; Jäger, Alexander; Weimer-Jehle, Wolfgang (2007): Leitbild Nachhaltigkeit. Eine normativ-funktionale Konzeption und ihre Umsetzung. Wiesbaden: VS-Verlag.

Schreurs, Miranda; Steuwer, Sybil D. (2015): Autonomous driving. Political, legal, social and sustainability dimensions. In: Markus Maurer, Christian Gerdes, Barbara Lenz und Hermann Winner (Hg.): Autonomes Fahren. Technische, rechtliche und gesellschaftliche Aspekte. Heidelberg: Springer, S. 151-173.

Sonnberger, Marco; Deuschle, Jürgen (2014): Maßnahmen zur Eindämmung von Rebound-Effekten im Wohn- und Mobilitätsbereich. Ergebnisse aus zwei Expertenworkshops. In: Stuttgarter Beiträge zur Risiko- und Nachhaltigkeitsforschung 31. Stuttgart: Universität Stuttgart.

Spiegel Online (2016): Tödlicher Unfall in USA. Wie gefährlich sind selbstfahrende Autos? Online verfügbar unter www.spiegel.de/auto/aktuell/tesla-diewichtigsten-fakten-zum-toedlichen-unfall-in-den-usa-a-1100803.html, zuletzt geprüft am 11.06.2018.

Spiegel Online (2018 a): USA. Tödlicher Unfall mit selbstfahrendem Uber-Auto. Online verfügbar unter www.spiegel.de/wirtschaft/unternehmen/ubertoedlicher-unfall-mit-selbstfahrendem-auto-a-1198884.html, zuletzt geprüft am 11.06.2018.

Spiegel Online (2018 b): Nach tödlichem Zusammenprall. US-Behörde untersucht Unfall von Tesla-Fahrzeug. Online verfügbar unter www.spiegel.de/ auto/aktuell/us-behoerde-untersucht-unfall-von-tesla-fahrzeug-aktiefaellt-a-1200209.html, zuletzt geprüft am 11.06.2018.

Spiegel Online (2018 c): Tödlicher Uber-Unfall. Fürchtet euch ruhig. Online verfügbar unter www.spiegel.de/auto/aktuell/uber-unfall-die-furcht-vorder-kuenstlichen-intelligenz-am-steuer-a-1199188.html, zuletzt geprüft am 11.06.2018.

Stuttgarter Nachrichten (2016): Kommentar zum Tesla-Unfall. Die Risiken der Roboter-Autos. Online verfügbar unter www.stuttgarter-nachrichten.de/ inhalt.kommentar-zum-tesla-unfall-die-risiken-der-roboterautos.36129819a9bf-4e7b-aa25-d1bd0bedf42e.html, zuletzt geprüft am 11.06.2018.

Tagesschau (2018 a): Autonomes Fahren: Die Angst vor dem Autopiloten. Online verfügbar unter www.tagesschau.de/wirtschaft/autonomes-fahren-109.html, zuletzt geprüft am 11.06.2018.

Tagesschau (2018 b): Bericht zu tödlichem Unfall. Software erkannte Fußgängerin zu spät. Online verfügbar unter www.tagesschau.de/wirtschaft/uberunfall-101.html, zuletzt geprüft am 11.06.2018.

Tagesschau (2018 c): Unfall mit Autopilot: Tesla kracht in Polizeiauto. Online verfügbar unter www.tagesschau.de/ausland/tesla-unfall-113.html, zuletzt geprüft am 11.06.2018.

Tagesschau (2018 d): Ihre Meinung zu: Tödlicher Unfall. Uber-Software erkannte Fußgängerin zu spät. Online verfügbar unter https://meta.tagesschau.de/ id/134499/toedlicher-unfall-uber-software-erkannte-fussgaengerin-zu-spaet, zuletzt geprüft am 11.06.2018.

Tagesschau (2018 e): Ihre Meinung zu: Unfall mit Autopilot. Tesla kracht in Polizeiauto. Online verfügbar unter https://meta.tagesschau.de/id/134671/ unfall-mit-autopilot-tesla-kracht-in-polizeiauto, zuletzt geprüft am 11. 06.2018.
Thomopoulos, Nikolas; Givoni, Moshe (2015): The autonomous car. A blessing or a curse for the future of low carbon mobility? An exploration of likely vs. desirable outcomes. In: European Journal of Futures Research 3 (1), S. 1-14.

TÜV Rheinland (Hg.) (2018): TÜV-Rheinland-Studie zur Sicherheit autonomer Fahrzeuge. Fahrer wollen jederzeit eingreifen können. Repräsentative Befragung von Autofahrern in Deutschland, USA und China (Pressemitteilung). Online verfügbar unter https://www.tuv.com/de/deutschland/ueber_uns/ presse/meldungen/newspdfde_358792.jsp, zuletzt geprüft am 11.06.2018.

Umweltbundesamt (Hg.) (2005): Qualitätsziele und Indikatoren für eine nachhaltige Mobilität. Dessau: Umweltbundesamt.

Verband der Automobilindustrie (Hg.) (2015): Automatisierung. Von Fahrerassistenzsystemen zum automatisierten Fahren. Berlin: VDA.

Vereinte Nationen (1992): Rio-Erklärung über Umwelt und Entwicklung, Rio de Janeiro. Online verfügbar unter www.un.org/depts/german/conf/agenda21/ rio.pdf, zuletzt geprüft am 27.4.2018.

Wadud, Zia; MacKenzie, Don; Leiby, Paul (2016): Help or hindrance? The travel, energy and carbon impacts of highly automated vehicles. In: Transportation Research 86, S. 1-18.

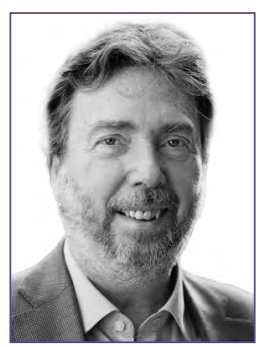

\section{DR. JÜRGEN HAMPEL}

ist akademischer Mitarbeiter am Lehrstuhl für Technik- und Umweltsoziologie der Universität Stuttgart und Mitglied des Zentrums für Interdisziplinäre Risiko- und Innovationsforschung der Universität Stuttgart. Seine Arbeitsschwerpunkte umfassen die soziologische Technik-, Risiko- und Umweltforschung, insbesondere die sozialwissenschaftliche Analyse gesellschaftlicher Reaktionen auf neue Technologien und dabei auftretender Konflikte.

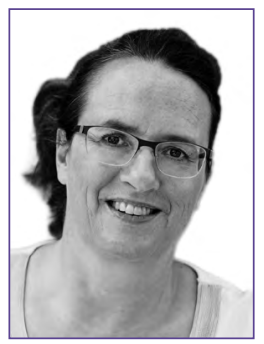

\section{PROF. DR. CORDULA KROPP}

ist Inhaberin des Lehrstuhls für Technik- und Umweltsoziologie und Direktorin des Zentrums für interdisziplinäre Risiko- und Innovationsforschung der Universität Stuttgart. Zu ihren Arbeitsgebieten zählen die Umwelt-, Technik- und Risikosoziologie, Forschung zum Wandel der Infrastruktursysteme in modernen Gesellschaften sowie Transformationsprozesse und soziale Innovation.

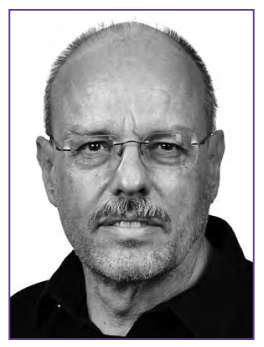

\section{DR. MICHAEL M.ZWICK}

arbeitet als Technik- und Umweltsoziologe am Institut für Sozialwissenschaften der Universität Stuttgart. Zu seinen Arbeitsschwerpunkten zählen neben der Umwelt- und Risikoforschung die Wahrnehmung des technischen Wandels in der Öffentlichkeit sowie qualitative und quantitative Methoden der empirischen Sozialforschung. 


\title{
The vision of delivery drones
}

\author{
Call for a technology assessment perspective
}

\author{
Michael Nentwich, Institute of Technology Assessment (ITA) of the Austrian Academy of Sciences (ÖAW), \\ 1030 Vienna, Apostelgasse 23 (mnent@oeaw.ac.at), (1) orcid.org/0000-0003-2269-0076 \\ Delila M. Horváth (delila.horvath@gmail.com), (1) orcid.org/0000-0003-4112-8070
}

The vision of drone-based delivery is not without presuppositions. To fully realize this vision, it is imperative to overcome the many technical and regulatory obstacles. Given the considerable depth of engagement of this technology - the airspace around us, which has so far only been used by birds and the occasional helicopter, would change profoundly - a number of typical technology assessment (TA) questions are on the table: Are there any safety concerns? Are there any environmental risks? Could criminals or terrorists misuse the technology? Are we going to face societal conflict in view of divergent interests? Is the current regulatory framework sufficient, or do we need new rules? This article provides a broad outline of this topic, gives first answers to the above mentioned questions, and concludes that an encompassing participatory TA study is needed.

\section{Vision Lieferdrohnen}

Aufruf zur Betrachtung aus Perspektive der Technikfolgenabschätzung

Die Vision eines drohnenbasierten Lieferverkehrs ist nicht voraussetzungslos. Viele regulative und technische Hürden müssen noch genommen werden, um sie Wirklichkeit werden zu lassen. Aufgrund der großen Eingriffstiefe dieser Technologieentwicklung - immerhin würde sich der uns umgebende Luftraum, der bislang nur von Vögeln und gelegentlichen Hubschraubern benutzt wird, gravierend ändern - stellen sich eine Reihe von typischen Fragen der Technikfolgenabschätzung (TA): Bestehen Sicherheitsbedenken? Gibt es Umweltrisiken? Kann die Technologie für kriminelle oder terroristische Zwecke missbraucht werden? Besteht ein gesellschaftliches Konfliktpotenzial angesichts unterschiedlicher Interessen? Reicht die aktuelle Regulierung aus oder müssen neue Regeln geschaffen werden? Dieser Artikel stellt das Thema in groben Zügen dar, gibt erste Antworten auf die genannten Fragen und erkennt die Notwendigkeit einer umfassenden, partizipativen TA-Studie.

Keywords: delivery drones; transport market; environmental, health and safety issues; ethical, legal, societal impacts

This is an article distributed under the terms of the Creative Commons Attribution License CCBY 4.0 (https://creativecommons.org/licenses/by/4.0/)

https://doi.org/10.14512/tatup.27.2.46

Submitted: 07. 03.2018. Peer reviewed. Accepted: 11. 05.2018

\section{Introduction}

Companies worldwide imagine a world in which everyday commodities will be delivered by drones through the air, and this emerging market for drone delivery is expanding. Following initiatives of small enterprises, for instance delivering pizza in New Zealand, large corporations as diverse as Amazon, Google (Project Wing), DHL, Mercedes-Benz and many others have started to invest a lot of resources in testing delivery by drones and have been lobbying for making this service a reality. Numerous start-ups and postal operators are launching delivery services by drones all around the word, in particular in Asia and Africa, testing the market and the legislative frameworks with this novel approach (for a market overview see Nentwich and Hórvath 2018, chapter 4).

This vision of drone-based delivery is not without presuppositions though. To realize the vision of drone-based delivery, many technical, regulatory and societal questions would have to be solved. Given the considerable depth of engagement - considering that the airspace around us, which has so far only been used by birds and the occasional helicopter, would change profoundly - a number of typical technology assessment (TA) questions are on the table: Are there any security concerns? Are there any environmental risks? Could criminals or terrorists misuse the technology? Are we going to face societal conflict given that divergent interests are involved? Does the current regulatory framework suffice or do we need new rules?

This article is based on an explorative report (Nentwich and Hórvath 2018) expanding on previous literature (Moe 2013; ITA 2014; POST 2014; Rao et al. 2016; Krieger-Lamina and Nentwich 2016). It gives first answers to the above-mentioned questions and is mainly based on an extensive Internet and literature search, several interviews with experts, and analytical considerations.

\section{Technical and legal preconditions}

There are two important preconditions for the launch of delivery services by drones: it has to be technically feasible and safe, and the law must allow autonomous flights in principle. Drone 
delivery seems only reasonable when they can be operated in an autonomous mode - except for very special scenarios (e. g. occasional fast delivery to remote areas). Otherwise, the main reasons for launching such service (e. g. cost reduction, automation, and speed) would be compromised if pilots were needed for each drone. However, to operate a drone autonomously presents technical challenges.

The main challenge regarding the operation of autonomous delivery drones is the development of robust sense and avoid ( $\mathrm{S} \& \mathrm{~A}$ ) technology. This term refers to a drone's capability to take-off, fly and land at the intended location and in the intended manner without colliding on the way. In order to do so, the drone needs a) a geo-location device which operates continuously and
The other main obstacle is the present state of regulation. Evidently, delivery drones do not belong to the category of lightweight flying objects (to which toy equivalents belong) because they need to carry a load in addition to the equipment needed for autonomous flying (such as cameras and other sensors, etc.). Consequently, delivery drones could only be employed under special conditions (e. g. licence). For on-board cameras and other equipment, a special additional licence granted by the aeronautical or aviation authorities would be required. However, so far, the law does not cover autonomous drones and therefor does not permit their use in Europe. In addition, under current regulation authorities allow the flight of delivery drones over populated urban areas, only on a case-by-case basis, which is obvi-

\section{To realize the vision of drone-based delivery, many technical, regulatory and societal questions would have to be solved.}

accurately, b) clear vision with the help of cameras (or radar), and c) well-developed algorithms to execute accurate landing. Moreover, delivery drones would need to exhibit technical readiness to overcome challenges caused by the weather, physical obstacles on the way (tall buildings, electric poles, cables, flying birds, other drones, and not least humans), and internal malfunctions. It has not yet been established which S \& A technologies (or which combination) would be the most reliable and cost-efficient. In particular, both the development and testing of Global Positioning Service (GPS), radar, infrared and other technologies are still ongoing. To this day, the threat of drones crashing (into another object or into each other) remains a challenge, which technicians have yet to solve satisfactorily. Furthermore, delivery drones would need to be able to respect the designated no-fly zones (geo-fencing), i. e. they need to have up-to-date access to the respective databases or air signals. In addition, drones must be able to autonomously and constantly communicate with and coordinate other air traffic and air control. Finally, battery capacity and thus reach (the distance a drone could cover) are additional important factors to consider, as are cargo weight restrictions. An Austrian pilot project in summer 2017 showed that flights with parcels weighing $3.5 \mathrm{~kg}$ could be transported for a distance of up to $10 \mathrm{~km}$ (Futurezone 2017). Most technicians seem optimistic that all of these challenges can soon be overcome, with some experiments and pilot tests already confirming this, others, however, disconfirm (Futurezone 2018). Our interim conclusion is that several technical aspects of autonomous drones still need further development and a few years of testing before it would be safe enough to launch extensive commercial delivery services - similar to driverless cars in general. Furthermore, functioning infrastructure, in particular landing spots, must be established. Several options are currently under development, including small movable platforms on a cantilever next to the window (e.g. http://win-port.de). ously not suitable for a business model relying on regular deliveries. As a result, legal frameworks would have to be adapted for delivery drones, for instance with an additional drone category with special rules. Subsequently, there would be a need for regulation in order to enable mass deployment, especially should delivery services by drones be authorised in urban areas. The regulatory debate has been under way for a couple of years (AAE/3AF 2015, p. 52 ff.) but is still open, in particular in respect to autonomous flying ${ }^{1}$.

\section{Possible applications}

The preliminary assessment of the possible impact of the introduction of commercial delivery drones outlined in the next sections refers to two potential applications as reference points. Based on the assumption that the two above-mentioned preconditions have been met, these applications have been extrapolated from the various experiments and activities from start-ups and major players and are thus meant to represent their visions of the future.

The first application concerns the widespread delivery of all kinds of small goods (parcels) by drones instead of (or in combination with) delivery vans and trucks. In this scenario - we will call it, non-technically speaking, the "pizza scenario" -, drones would be fulfilling the function of the so-called "last mile delivery": various logistics providers would use the urban (and rural) airspace on a regular basis to deliver goods to consumers. The idea behind this would be to be able to deliver even faster

1 See the current proposals by the European Aviation Safety Agency (EASA): easa.europa.eu/easa-and-you/civil-drones-rpas/drones-regulatory-frameworkbackground; and the respective regulatory process at EU level where a political agreement seems to have been reached at the end of 2017 (easa.europa.eu/ document-library/opinions/opinion-012018). 
than presently possible, in particular given the traffic jams on the ground. "Amazon Prime Air" is one example of this business model, but it is not yet operative. There are, however, many other examples for delivery services for food and other commodities worldwide, for instance: pizza in New Zealand, fast food in Iceland, or coffee in Switzerland and Dubai (Nentwich and Hórvath 2018, p. 24 ff.).

The second application - we will call it the "emergency scenario" - is less encompassing: the delivery service by drones would take place only in one or several niche markets. The commodities transported by the drones could be special goods in the medical field - like blood supplies, which would be routinely transported between hospitals, pharmacies, and practitioners -, or other emergency goods. ${ }^{2}$ Another potential niche market could be the regular supply of goods to remote areas to where there are no roads or where no other connection exists during specific seasons (for instance in rural Africa or in alpine regions). For an overview, see Nentwich and Hórvath (2018, p. 26).

\section{Potential impacts of employing drones for deliveries}

Amongst the varied potential impacts of drone deliveries, this short article will only feature a selection and includes the following issues: changes in consumer behavior, resilience of the delivery system, protection against misuse. For a complete overview, though not an in-depth analysis, see Nentwich and Hórvath (2018).

\section{Health, safety and security}

There are two types of health risks resulting from accidents. First, malfunction of the navigation system, in particular in bad atmospheric conditions, may lead to accidents. Especially in urban areas with a dense population, collisions of drones with humans are possible, and injuries quite likely as the rotors are sharp and loaded drones weigh several kilograms. As long as delivery drone systems have not yet been put in place, and depending on the type of application, estimating the likelihood of accidents is presently still difficult.

Another potential health risk stems from the drone's load. In the event of a crash of a drone carrying a dangerous good, various scenarios with far-reaching consequences are conceivable. The definition of what counts as dangerous goods would have to be refined: for instance one may question whether blood samples of ill people, medical probes or vaccines should be included in such definition as these could potentially contaminate the environment or pose a direct threat to people. There may even be the possibility of prohibiting the transport of dangerous goods altogether.

2 For instance, Zipline Int. delivers blood samples and medical supplies, flyzipline.com; Matternet specialises in emergency goods, mttr.net.
Furthermore, drones disguised with the logo of a delivery firm could easily be misused for illegal purposes, e.g. trafficking (drugs, weapons), spying, carrying bombs, or the drone itself could be used as a kamikaze weapon (e. g. AAE/3AF 2015, p. 49 f.).

\section{Environment}

As drones use the environment (both natural and man-made), they are also a potential risk to it. The first concern is the drones' effect on wildlife, and birds in particular. When drones intrude into the habitat of animals, there is a double risk: either the animals may be harmed, or they could be a threat to the effective operation of drones. With regard to the latter, such a scenario has already been documented in Austria when eagles mistook drones for food (The Independent 2015). Regarding the former, there are concerns that the safety of birds could be at risk because of the possibility of collision, quite similar to the risk associated with wind turbines. It must be noted that not only wildlife could be affected: depending on the territory the drones would be allowed to fly through, they could have an impact on various domesticated animals (pets and farm animals) as well.

Even without the risk of collisions, the drones' noise and frequent presence in the animals' habitats may be a stressor for them, similar to nearby roads. The effect of noise produced by drones on wildlife has not been studied yet, but serious concerns exist and should be taken into consideration. Several aspects of must be considered: altitude, flight corridors, no-fly zones, and landing sites.

Another possible environmental risk is debris. Regardless whether debris results from collision or from forgotten or abandoned goods, the question of waste poses another series of challenges. Consequently, this raises questions regarding responsibility and actual response: who would be responsible for cleaning up debris, and who would bear the costs of damage or compensation? This issue prompts comparison with fly tipping. Here, too, the question regarding taking responsibility remains difficult to answer.

\section{As drones use the natural}

\section{and man-made environment,}

\section{they are also a potential risk to it.}

From a TA perspective, another environmental aspect to consider is energy consumption. Drones need electricity, and although each individual flight would not consume much, the overall picture of a universal drone delivery system may be different, in particular when compared with current deliveries by car (possibly electric vans in the future) in which many parcels can be transported at once. In a recent research article, Stolaroff et al. (2018) conclude that for parcels up to $0.5 \mathrm{~kg}$ the energy 
balance is in favour of drones. However, the overall assessment may be different when putting the entire infrastructure into perspective. Overall, a serious assessment of the ecological footprint (life-cycle assessment) is necessary, including - amongst other factors - the lifetime of drone batteries.

\section{Labour market}

The technology of drones and its potential effect on the job market are another area of concern (OECD 2015). The transport and logistics industry employs large numbers of drivers to deliver parcels in person on the so-called "last mile". With the advent of 24/7 online shopping, the market segment of delivering to customers' homes has increased considerably, as has the labour market for parcel pickers and delivery van driver. If the use
As the process of automation would result in less need for human workers, the group of unskilled workers could suffer most (Forbes 2017; Goldman Sachs o. J.; Reuters 2017).

\section{Ethics}

What behaviour will be pre-programmed into the software of drones for the scenario of an impending, no longer avoidable accident? Similar to algorithms within the context of driverless cars, drones must be able to make a number of decisions in split seconds prior to an event in order to remain operative. For driverless cars, these tricky issues have already seen extensive discussion among ethicists and technologists (Maurer et al. 2015). A similar discussion for drones is still missing (for a first contribution see Luppicini and So 2016).

\section{Now is the perfect time for carrying out an encompassing technology assessment study on drones - and to induce a public debate about it with all concerned stakeholders as well as citizens.}

of drones for the last mile should become more common, employment of delivery drivers will eventually shrink.

Such effects would depend on the specific type of application and the delivery modes that have been put in place. Evidently, the labour market for delivery drivers in the pharmaceutical industry is much smaller than in the consumer goods sector. In cases where drones take off from automated intermediate storage facilities, van drivers would be out of business in the long run (except for when it comes to large and/or heavy parcels that could not be carried by drones). If drones should take off from delivery vans, there would still be a need for drivers - unless those vans would eventually become driverless cars themselves. Still, their numbers would be reduced because using drones for the last mile is purportedly much more time efficient: not only are drones faster than a human walking up the stairs, more drones could be taking off from one van at the same time. This would lead to faster turnover times for delivery vans in which there would still be only one driver.

To the authors' knowledge, no specific scientific study on last mile delivery has yet been published. However, several studies examine the process of digitalisation and automation in various job markets in different countries (EPTA 2016; Frey and Osborne 2013; Čas et al. 2017). Economists are split over the exact figures, but agree in predicting huge job losses for the general transport sector (taxi drivers, lorry drivers, etc.). Further research is needed.

It is also important to acknowledge that elementary occupations, serve as a social and economic safety net for those individuals who lack an education or vocational/specific training.
The typical and often quoted scenarios, in which some kind of damage is unavoidable, raise different ethical concerns over drone behaviour. For instance: what if a drone which carries a life-saving cargo can avoid crashing into a human only by abandoning or destroying the parcel that could have saved someone else's life (e. g. a parcel containing a human organ for transplant). Such ethical question and similar ones would have to be answered beforehand, with relevant drone behaviour responses being programmed into the software. Similar to the discussion about autonomous cars, the question arises whether it should be the programmer who decides in advance, the drone manufacturer, the delivery service enterprise, the sender or the recipient of the parcel, or rather society at large and thus the legislator. How would the implementation of these decisions on drone behaviour be overseen? Clearly, answering all these questions is not easy (Krieger-Lamina and Nentwich 2016). For the drone to be able to take whichever decision, permanent surveillance and analysis of the environment is a precondition.

Incidentally, this ethical dilemma is also present in the case of a delivery service being restricted to emergency parcels (the second type of application, i. e. our "emergency scenario"). Taking into consideration that an emergency represents a situation where decision-making is difficult, the question of what constitutes an emergency and what does not remains unanswered. The delivery of organs for transplant may safely be considered an emergency, but what about other cases such as the transport of medical probes and blood samples?

In the second type of application, one may even go one step further: would drone delivery services in urban settings be the 
privilege of medical entities only? If so, what counts as a medical entity? Would there be strict regulation of who is allowed to fly and who is not, and how to limit and punish misuse of privileges? If strict controls, time restrictions, flight corridors etc. were in place, such service would be a scarce commodity, probably incurring higher prices. For instance, a certain patient's blood could be given priority because she or he paid extra fees to obtain results faster. This is a typical TA dilemma, which could be termed the "drones' divide": in one scenario only the rich might be in a position to afford it (yet would still use common/ shared airspace), which may lead to an additional difference in the quality of healthcare. The same set of questions may also be asked for other services.

\section{Further potential areas of societal conflict}

At least two major issues have the potential to raise the public's concern and to cause societal conflict: noise pollution and negative aesthetic impact on airspace: Like residents near roads or airports, residents living directly under the air corridors for regular drone delivery flights could also suffer from noise pollution. It is noteworthy to state that with our first application scenario, the pizza scenario, heavy drone traffic would develop over time. Whilst a single drone with eight electrical mini engines is not very noisy (unless it is very near), many of them at the same time certainly would be. Even if it were assumed that later generations of drones emit less noise, there would still be a limit to further improvements, as it is unlikely that airflow around the

\section{Many questions remain unanswered: they concern the commercial use of drones, the technical, legislative and societal aspects as well as safety and environmental risks.}

\section{Protection of privacy}

If parcel delivery by drones becomes a (legally) accepted service, the technology has the potential to affect and possibly conflict with privacy. Today, even without large numbers of drones in the air, this has already become an issue. Many toy drones in particular are equipped with cameras, even though this violates the law, unless a specific licence has been obtained, thereby alarming neighbours when the drone flies over one's land or approaches one's balcony. Numerous articles exist in the mass media addressing the issue (see for example Consumer Reports 2016; The Next Web 2017; Recode 2017), and several companies offer anti-drone devices, like drone guns, for self-help "law enforcement" purposes. Dutch police apparently trains falcons for drone hunting (Futurezone 2016).

Although taking pictures, filming or audio surveillance is not amongst the main purposes of delivery drones, they still need to sense where they are going (and constantly survey their environment). Consequently, they are equipped with technology that can "see", helping the drone with orientation. The data generated by these sensors during the flight could be deleted immediately. However, it may be legally required to keep a record of a flight, e.g. to prove the drone has not caused any damage or has observed aviation laws. Such a scenario seems likely as it parallels similar developments in other areas, e. g. black boxes in airplanes, tachographs in lorries and similar on-board recording devices in cars in the near future. Such devices generate, record and store video material coupled with location data and time stamps. All this data can be quite sensitive should people be identifiable from the recordings. Birds'-eye views from private properties, too, may be sensitive, as are visual recordings of public buildings (e. g. parliament buildings) or military facilities. many rotors can be avoided. This is similar to the noise produced by car tyres, which is, together with the airflow around the car's body, louder than that of the engine when travelling above a certain speed. Hence, even the most noise reduced electrical engines cannot ensure altogether silent e-cars or drones.

Besides evident noise pollution along highly used air corridors, noise emittance through delivery manoeuvers close to customers, particularly in densely populated urban areas, should not be underestimated. Only the noise produced by the expected air traffic in the second application scenario, the emergency scenario, would be of minor concern. For the first application scenario, noise pollution can safely be deemed a problem, which, if unsolved, may lead to resistance in the population. This raises the question how society would decide where drones should or would be allowed to fly in order to reduce noise pollution. Nofly zones may be part of the solution, as would be corridors high above street level and away from buildings. However, is there any solution for the last mile, i. e. the surroundings of the prospective landing spots close to the customers?

In the same way that the aesthetics of power lines and wind parks have been questioned, the aesthetic of swarming drones in the lower airspace is likely be questioned by parts of the population. At first, this may sound like a luxury problem, and society has indeed accepted many similar compromises in the past: there are fewer untouched natural landscapes, and in urban areas, it has become the norm to utilize the ground level for traffic, street furniture and appliances. Furthermore, opinions on the aesthetic value of different types of buildings and infrastructure will always remain divided. However, the authors of this article assume that extensive use of currently empty airspace could well trigger public resistance against a new and qualitatively different exploitation of and intrusion in a common good. 


\section{Preliminary conclusions}

As can be seen, many questions regarding the commercial use of drones remain unanswered. They concern the technical, legislative as well as societal aspects, safety, and environmental risks. The answers to them will differ when the different application scenarios are considered. In our "pizza scenario" with omnipresent delivery drones forming part of everyday reality, the impact on society will evidently be more severe than in the second, more restrictive application scenario with deliveries only in special cases. Whilst implementing the so-called "emergency scenario" would still require considerable preparation, both in technical and legal terms, establishing such a niche market would probably face less opposition. By contrast, the first application scenario not only requires much more sophisticated infrastructure and regulation, it also raises some potentially controversial questions.

From a TA perspective, these questions cannot be answered without in-depth interdisciplinary examination. Our study only provides a snapshot or preliminary stocktaking of the issues involved; it can be expected that some of the points raised may prove less controversial or turn out to be unimportant, but more issues that are salient could well be discovered. Moreover, many of the identified issues concern core values, and the technology touches upon the private lives of a large number of individuals. To give a few examples: the relationship between economic interests and the protection of wildlife is not an obvious one; the question of the aesthetic consequences of mass-droning is difficult to answer in a top-down manner; noise is another issue where society constantly searches for compromises.

As the technology has almost matured and many commercial entities are readying themselves, now would be the time to open up debate and examine the possible opportunities and risks of such a service. Conducting a combined expert and stakeholder/lay participatory TA study is highly recommended. A participatory approach would allow for the inclusion of the informed views of citizens and those potentially affected, not

\section{We have a window of opportunity to}

\section{shape the path of drone development}

$$
\text { through anticipatory governance. }
$$

least because the topic involves value-based decisions. Consequently, the proposed study would try to answer the question which of the above-mentioned application scenarios (or any others that may arise) is wanted by society, and under what conditions?

On a final note, the authors of this article are certain that the timing for carrying out such an encompassing study now - and for the inclusion of a public debate about it - would be perfect.
Today, the technology may not yet be at its full potential, and commercial actors may not have invested heavily in their business models yet. To translate this into TA language: currently there is a window of opportunity to overcome the well-known control or Collingridge dilemma. Today, anticipatory governance aimed at shaping the technological and economic path is still an efficient and effective option.

\section{References}

AAE/3AF (2015): Present and future of civilian drones. AAE Dossier 40/3AF Cahier 16. Paris: Air and Space Academy/French Aerospace Society. Online available at http://espas.eu/orbis/sites/default/files/generated/document/en/D40_ Ebook_UK.pdf, last accessed on 09.05.2018.

Čas, Johann; Rose, Gloria; Schüttler, Lisa (2017): Robotik in Österreich. Kurzstudie Entwicklungsperspektiven und politische Herausforderungen. Vienna: ITA. Online available at http://epub.oeaw.ac.at/ita/ita-projektberichte/2017-03. pdf, last accessed on 09.05.2018.

Consumer Report (2016): Drone privacy. is anyone in charge? Online available at https://www.consumerreports.org/electronics/drone-privacy-is-anyonein-charge/, last accessed on 14.05.2018.

EPTA - European Parliamentary Technology Assessment (2016): The future of labour in the digital era. Ubiquitous computing, virtual platforms, and real-time production. Vienna: ITA. Online available at http://epub.oeaw. ac.at/ita/ita-projektberichte/EPTA-2016-Digital-Labour.pdf, last accessed on 09.05.2018.

Forbes (2017): Should package delivery be automated with drones? A look at the pros and cons. Online available at https://www.forbes.com/ sites/quora/2017/04/17/should-package-delivery-be-automated-withdrones-a-look-at-the-pros-and-cons/\#236b19561bc9, last accessed on 14. 05.2018.

Frey, Carl B.; Osborne, Martin A. (2013): The future of employment: How susceptible are jobs to computerisation? Oxford: Martin Programme on the Impacts of Future Technology.

Futurezone (2016): Niederländische Polizei trainiert Adler für Drohnenjagd. Online available at https://futurezone.at/digital-life/niederlaendischepolizei-trainiert-adler-fuer-drohnenjagd/178.424.561, last accessed on 14. 05.2018

Futurezone (2017): TU Graz testet Drohnen als Paketzusteller. Online available at https://futurezone.at/science/tu-graz-testet-drohnen-alspaketzusteller/274.382.090, last accessed on 14. 05.2018.

Futurezone (2018): Video. Drohne der russischen Post knallt in Hauswand. Online available at https://futurezone.at/digital-life/video-drohne-der-russischenpost-knallt-in-hauswand/400014766, last accessed on 14.05.2018.

Goldman Sachs (o. .).: Drones. Reporting for work. Online available at http://www.goldmansachs.com/our-thinking/technology-driving-innovation/ drones, last accessed on 14.05.2018.

ITA (eds.) (2014): Drohnen - fliegende Alleskönner? ITA-Dossier no. 6. Vienna: Institute of Technology Assessment. Online available at http://www.austriaca. at/ita/ita-dossiers/ita-dossier006.pdf, last accessed on 09.05.2018.

Krieger-Lamina, Jaro; Nentwich, Michael (2016): Zivile Autonome Fahrzeuge zu Land, in der Luft und zu Wasser. TA16 "Smart New World - Was ist smart an smarten Technologien?" Vienna: ITA. Online available at https://www. oeaw.ac.at/ita/fileadmin/redaktion/Veranstaltungen/konferenzen/ta16/ ta16-krieger-lamina-nentwich.pdf, last accessed on 09.05.2018. 
Luppicini, Rocci; So, Arthur (2016): A technoethical review of commercial drone use in the context of goverance, ethics, and privacy. In: Technology in Society 45, p. 109-119.

Maurer, Markus; Christian, Gerdes; Lenz, Barbara; Winner, Hermann (2015): Autonomes Fahren. Technische, rechtliche und gesellschaftliche Aspekte. Wiesbaden: Springer Vieweg.

Moe, Åke Refsdal (2013): Take-off for civilian drones. Blog. Online available at http://teknologiradet.no/english/take-off-for-civilan-drones, last accessed on 09.05.2018.

Nentwich, Michael; Hórvath, Delila Mercédesz (2018): Delivery drones from a technology assessment perspective. Overview report, No.2018-01. Vienna: ITA. Online available at http://www.austriaca.at/0xc1aa5576_0x00377231.pdf, last accessed on 09. 05.2018.

OECD - Organization of Economic Co-operation and Development (2015): Employment outlook 2015. Paris. DOI: 10.1787/empl_outlook-2015-en.

POST (2014): Civilian Drones. POST Notes No. 479, London: Parliamentary Office of Science and Technology. Online available at http://researchbriefings.files. parliament.uk/documents/POST-PN-479/POST-PN-479.pdf, last accessed on 14. 05.2018

Rao, Bharat; Goutham Gopi, Ashwin; Maione, Romana (2016): The societal impact of commercial drones. In: Technology in Society 45, p. 83-90.

Recode (2017): Federal privacy laws won't necessarily protect you from spying drones. Online available at https://www.recode.net/2017/3/15/14934050/ federal-privacy-laws-spying-drones-senate-hearing, last accessed on 14. 05.2018 .

Reuters (2017): U.S. commercial drone use to expand tenfold by 2021. Online available at https://www.reuters.com/article/us-usa-drones/u-scommercial-drone-use-to-expand-tenfold-by-2021-government-agencyidUSKBN16S2NM, last accessed on 14.05.2018.

Stolaroff, Joshuah; Samaras, Constantine; O’Neill, Emma; Lubers, Alia; Mitchell, Alexandra; Ceperley, Daniel (2018): Energy use and life cycle greenhouse gas emissions of drones for commercial package delivery. In: Nature Communications 9 (1), p. 409. Online available at https://www.nature.com/articles/ s41467-017-02411-5.pdf, last accessed on 14.05.2018.
The Independent (2015): Two eagles mistake a drone for food in Austria. Online available at https://www.independent.co.uk/video/News/two-eaglesmistake-a-drone-for-food-in-austria-a6733351.html, last accessed on 14.05. 2018.

The Next Web (2017): Amazon patent details the scary future of drone delivery. Online available at https://thenextweb.com/tech/2017/08/24/amazonpatent-details-the-scary-future-of-drone-delivery, last accessed on 14.05.2018.

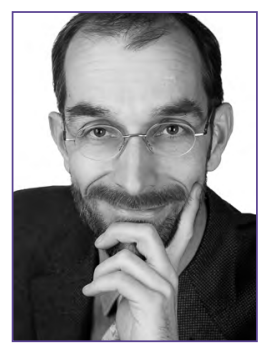

\section{PD DR. MICHAEL NENTWICH}

holds a Ph. D. in law and is director of the Institute of Technology Assessment (ITA) of the Austrian Academy of Sciences (ÖAW) in Vienna (since 2006). He is a passionate technology assessor (since 1987) as well as science and technology scholar and teacher.

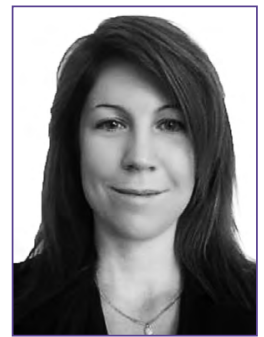

\section{DELILA M. HORVÁTH}

is a sociologist with a Master degree in Science and Technology Studies and is a member of the Space Generation Advisory Council.

\section{Nachhaltigkeit}

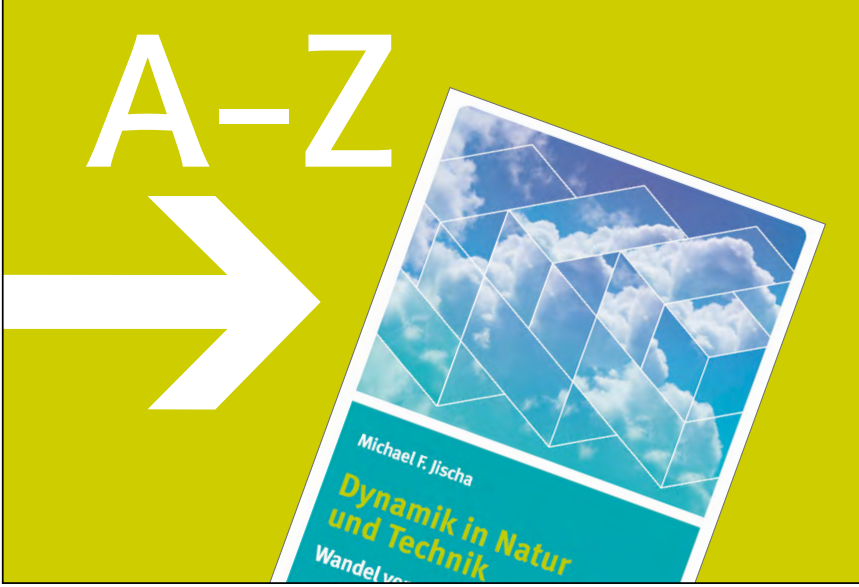

\section{wie Naturgesetze}

Warum kann ein Elefant einen Artgenossen nicht überspringen, während dies einem Hasen leicht gelingt? Was können wir von der Natur lernen - und können wir etwas gegen den Rückgang der Artenvielfalt tun? Diese und viele weitere Fragen können mit Blick auf die Naturgesetze beantwortet werden. Michael Jischa untersucht, wie sich unser Leben in Zeiten des technikgetriebenen, beschleunigten Wandels verändert - und er zeigt auf, vor welchen Herausforderungen wir heute stehen.

\section{F. Jischa}

\section{Dynamik in Natur und Technik}

Wandel verstehen und gestalten

348 Seiten, broschiert, 28,- Euro, ISBN 978-3-96238-040-3

Erhältlich im Buchhandel oder versandkostenfrei innerhalb Deutschlands bestellbar unter www.oekom.de 